\title{
TWO NEW TALL HERB COMMUNITIES WITH THE DOMINANT LASERPITIUM SILER AND GRAFIA GOLAKA FROM THE SE ALPS (NE ITALY, W SLOVENIA)
}

\author{
Igor DAKSKOBLER $^{1} \&$ Livio POLDINI ${ }^{2}$
}

\begin{abstract}
The article describes two new tall herb communities in the Southeastern Alps, Centaureo julici-Laserpitietum sileris and Laserpitio sileri-Grafietum golakae. Both are long-term successional stages in the overgrowing of abandoned hay meadows in the altimontane and subalpine belt of the southern and southwestern Julian Alps (including the Julian Prealps), partly also the Carnic Alps, that have been abandoned for over 50 years. In the succession sere where the altimontane-subalpine Illyrian beech forest (Dentario pentaphylli-Fagetum, Ranunculo platanifolii-Fagetum, Polysticho lonchitis-Fagetum) is the potential natural vegetation on steep, sunny limestone (rarely dolomite) slopes, the species Laserpitium siler and (or) Grafia golaka act as inhibitors also due to their high allelopathic potential; in turn, spontaneous afforestation with spruce and some deciduous trees has been slow and gradual.
\end{abstract}

Key words: Laserpitium siler, Grafia golaka, synsystematics, syndynamics, the Julian Alps, the Carnic Alps, Slovenia, Italy, Natura 2000.

\section{Izvleček}

Opisujemo dve novi združbi visokih steblik v Jugovzhodnih Alpah, Centaureo julici-Laserpitietum sileris in Laserpitio sileri-Grafietum golakae. Obe sta dolgotrajen sukcesijski stadij pri zaraščanju nekdanjih in že več kot 50 let opuščenih senožeti v altimontasnkem in subalpinskem pasu južnih in jugozahodnih Julijskih Alp (vključno z Julijskimi Predalpami) in deloma tudi Karnijskih Alp. V sukcesijskem nizu, v katerem je potencialno naravna vegetacija na strmih prisojnih apnenčastih (redkeje dolomitnih) pobočjih altimontansko-subalpinski ilirski bukov gozd (Dentario pentaphylli-Fagetum, Ranunculo platanifolii-Fagetum, Polysticho lonchitis-Fagetum), vrsti Laserpitium siler in (ali) Grafia golaka tudi zaradi svojega močnega alelopatskega potenciala delujeta zaviralno in je zato zaraščanje s smreko in nekaterimi listavci počasno in postopno.

Ključne besede: Laserpitium siler, Grafia golaka, sinsistematika, sindinamika, Julijske Alpe, Karnijske Alpe, Slovenija, Italija, Natura 2000.

\section{INTRODUCTION}

Several years ago (Dakskobler 2003), we studied forest development on abandoned hay meadows in the southern Julian Alps. These hay meadows were cleared in the belt of the subalpine beech forest classified into the association Polysticho lonchitis-Fagetum and are gradually becoming overgrown, especially with spruce. Based on the floristic-phytosociological analysis of 23 relevés the species-rich former hay-meadows were classified into the southeastern-Alpine alliance Caricion austroalpinae Sutter 1962 and, being a relatively long-term successional stage, described as a provisional new association Centaureo julici-Laserpitietum sileris nom. prov. Subsequent research conducted in the various parts of the Julian Alps (the upper Bača Valley, the Breginjski Stol ridge, the valleys of Bala and Loška Koritnica) confirmed our decision for an independent association as

\footnotetext{
${ }^{1}$ Institute of Biology, Scientific Research Centre of the Slovenian Academy of Sciences and Arts, Regional unit Tolmin, Brunov drevored 13, SI-5220 Tolmin, Igor.Dakskobler@zrc-sazu.si

${ }^{2}$ Dipartimento di Scienze della Vita, Università di Trieste, via L. Giorgieri, 5 - Edificio Q (stanza 104, primo piano), I-34127 Trieste, Italia, poldini@units.it
} 
these stands form a physiognomically (in their appearance) and floristically distinct community that can be spatially delimited and is relatively long-standing due to the slow rate of spontaneous afforestation. Latest research (comp. Kaligarič et al. 2011) has shown a strong allelopathic potential of Laserpitium siler, which in addition to other factors (snowslides) inhibits successional development back towards forest. A brief report on similar fringe communities from the Italian part of the Julian Alps and the Carnic Alps was published several years ago (Poldini 2003). A comparison of the collected relevé material from the whole region (W Slovenia and NE Italy) facilitated the typification of two new associations, Centaureo julici-Laserpitietum sileris and Laserpitio sileri-Grafietum golakae.

\section{METHODS}

We inventoried the abandoned grasslands in the upper montane and subalpine belt (900 to $1900 \mathrm{~m}$ a.s.l.) in the western, southern and southwestern part of the Julian Alps (Figure 1) and partly also in the Carnic Alps. Phytosociological records were made according to the standard CentralEuropean phytosociological method (Braun-Blanquet 1964) and entered into the FloVegSi database (Seliškar et al. 1993). Relevés from the Slovenian part of the Julian Alps (some of which have already been published as a separate subassociation Centaureo julici-Laserpitetum sileris asphodeletosum albi nom. prov. - Dakskobler et al. 2007) were combined with the relevés of slightly similar stands in NE Italy: the Carnic Alps: M. Chiadin - Sappada, M. Talm, the western Julian Alps: Val Raccolana, the Julian Prealps: Mt. Matajur, the Stol ridge / Gran Monte: Punta Lausciovizza / Lanževica; M. Cuarnan / Mali Karman, the Muzci ridge / Cime del Monte Musi: M. Cadin. Combined cover-abundance values were transformed into numerical values (1-9) according to van der Maarel (1979). Numerical comparisons were performed with the SYN-TAX 2000 program package (Podani 2001). The relevés were compared by means of "(unweighted) average linkage method" - UPGMA, "Incremental sum of squarres method" - MISSQ and principal coordinates analysis (PCoA). Wishart's similarity

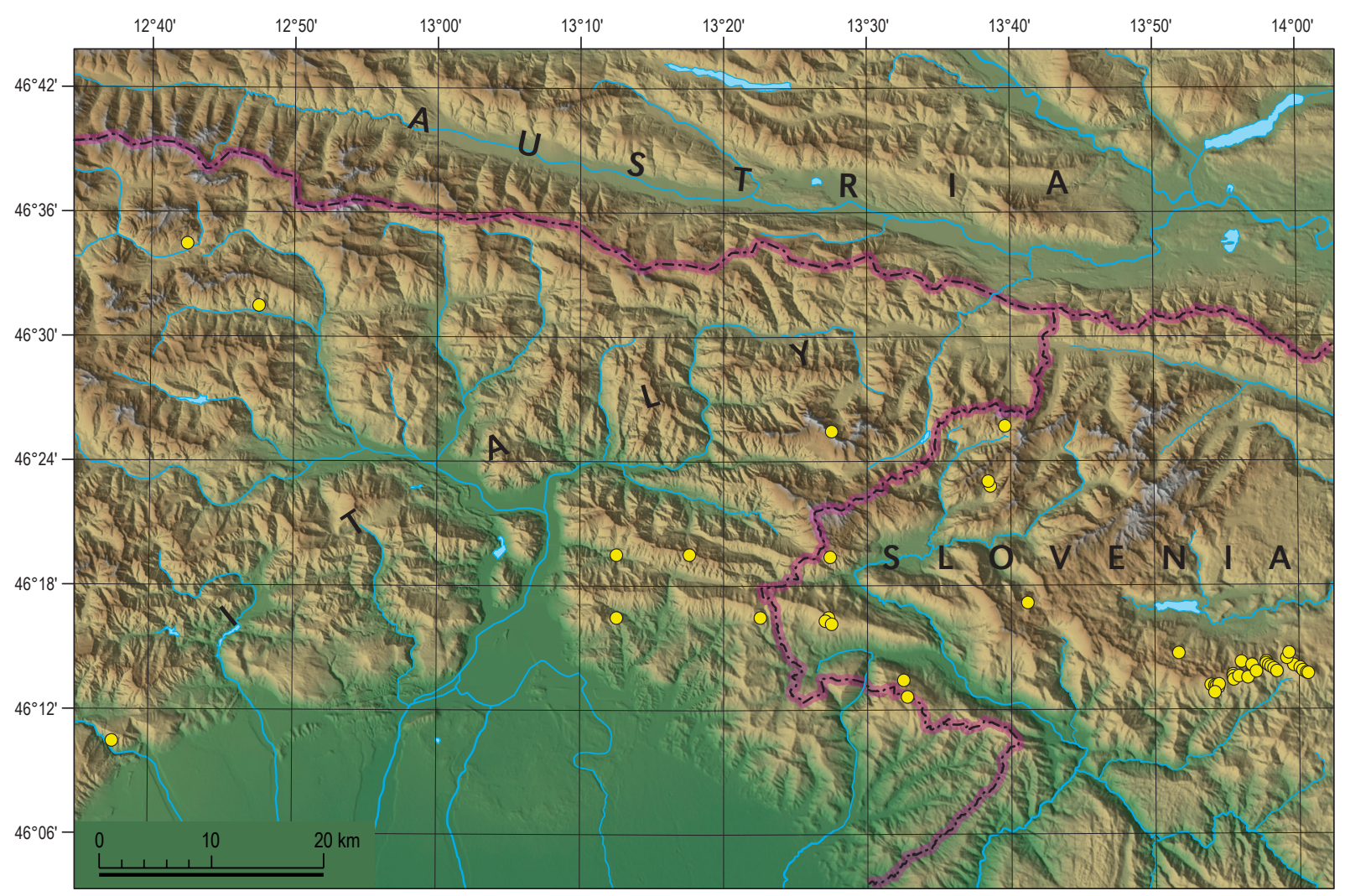

Figure 1: Research area with approximate localities of relevés in scale 1: 500000 (Map source: Jarvis \& al. 2008). Slika 1: Raziskovano območje s približno lokacijo popisov v merilu 1: 500000 (vir za zemljevid: Jarvis \& al. 2008). 
ratio was used in all of the methods. The nomenclature source for the names of vascular plants is the Mala flora Slovenije (Martinčič et al. 2007) - except for the taxa Knautia ressmannii (Pacher) Briq. and Bupleurum ranunculoides subsp. caricinum (DC.) Arcang. - and Martinčič (2003) for the names of mosses. The nomenclature of the syntaxa follows Šilc \& Čarni (2012). In the classification of species into phytosociological groups (groups of diagnostic species) we mainly refer to the Flora alpina (Aeschimann et al. 2004 a, b, c). Coordinates of relevés are determined according to the Slovenian geographic coordinate system D 48 (5th zone) on the Bessel elipsoid and with Gauss-Krüger projection.

\section{ECOLOGICAL DESCRIPTION OF THE STUDY AREA}

The predominant geological bedrock in the research area is limestone, in places admixed with marlstone and chert, rarely with dolomite. The soils are shallow fresh rendzinas, very rarely brown soils. Potential natural vegetation of the researched part of the Julian Alps is mainly beech forest classified into the associations Ranunculo platanifolii-Fagetum, Dentario pentaphylli-Fagetum and Polysticho lonchitis-Fagetum; the grasslands above the upper timberline are classified into the associations Avenastro parlatorei-Festucetum calvae and Ranunculo hybridi-Caricetum sempervirentis. The climate is relatively warm and very humid and mean annual precipitation usually exceeds 2500 $\mathrm{mm}$. The slopes are mainly sunny and open to the influence of the Adriatic Sea (for more information on the climate of the study area see Dakskobler 2003, Čušin 2006, Gobbo \& Poldini 2005).

\section{RESULTS AND DISCUSSION}

Firstly, we compared 87 relevés of stands with dominant Laserpitium siler and (or) Grafia golaka in the Julian Alps and their foothills (the so-called Julian Prealps) and the Carnic Alps Figures 2 and 3. Stands with the dominant Grafia golaka from the Julian Prealps (Mt. Matajur, the Stol ridge / Gran Monte and the Muzci ridge / Cime del Monte Musi) clearly separated from the stands with the dominant Laserpitium siler from the western and southern Julian Alps and the Carnic Alps. Four main groups in the den- drogram (Figure 2) were interpreted as: 1 Centaureo julici-Laserpitietum sileris typicum, 2 Centaureo julici-Laserpitietum sileris var. geogr. Pedicularis elongata, 3 Centaureo julici-Laserpitetum sileris asphodeletosum albi and 4 Laserpitio sileri-Grafietum golakae. Two-dimensional scatter-diagram (Figure 3) on the first axis partly indicates the phytogeographical gradient and on the second axis the altitudinal gradient. This comparison formed the basis for the analytic tables (Tables 1, 2, 3, 4). The first table comprises typical stands of the association Centaureo julici-Laserpitietum sileris, Table 2 encompasses fringe stands of this association which indicate possible transitions to similar tall herb and mountain grassland communities (for the time being treated as a variant Centaureo julici-Laserpitietum sileris typicum var. Brachypodium rupestre), the third table comprises subalpine stands with the dominant Laserpitium siler from NE Italy (Centaureo julici-Laserpitietum sileris var. geogr. Pedicularis elongata) and the fourth table the stands with the dominant Grafia golaka (Laserpitio sileri-Grafietum golakae).

The second step was to make a synoptic table (Table 5) into which we classified the above four syntaxa, the relevés of the subassociation Centaureo julici-Laserpitietum sileris asphodeletosum albi nom. prov. (Dakskobler et al. 2007, Tab. 1, relevés 8-33) and two similar fringe communities from central and southeastern Slovenia: Scabioso hladnikianae-Grafietum golakae Čarni 2007 from the Polhov Gradec Hills (Čarni 2007) and Laserpitietum sileris Springer 1987 var. geogr. Dianthus croaticus from the Gorjanci hills (Čarni et al. 2005). Some of the communities with the dominant $L a$ serpitium siler, e.g. Carici humilis-Centaureetum rupestris Ht. 1931 laserpitietosum sileris Kaligarič \& Poldini 1997 (Kaligarič 1997, Kaligarič \& Poldini 1997), Libanotido-Laserpitietum sileris van Gils et al. 1975 (van Gils et al. 1975, Čarni 1997), LaserpitioFestucetum alpestris Pedrotti 1970 (Pedrotti 1970), Origano-Calamagrostietum variae Lippert ex Thiele 1978 (Grabherr et al. 1993: 431-433) and Laserpitio-Seslerietum Moor 1957 (Oberdorfer 1978, Ewald 1996), were not included in our comparison as they are either communities of a different altitudinal belt (submontane, lower montane) and (or) communities of a phytogeographically entirely different environment and subsequently clearly floristically different.

Some of the species common to all of the compared communities are Laserpitium siler, L. latifolium, Polygonatum odoratum, Silene nutans s. lat., 


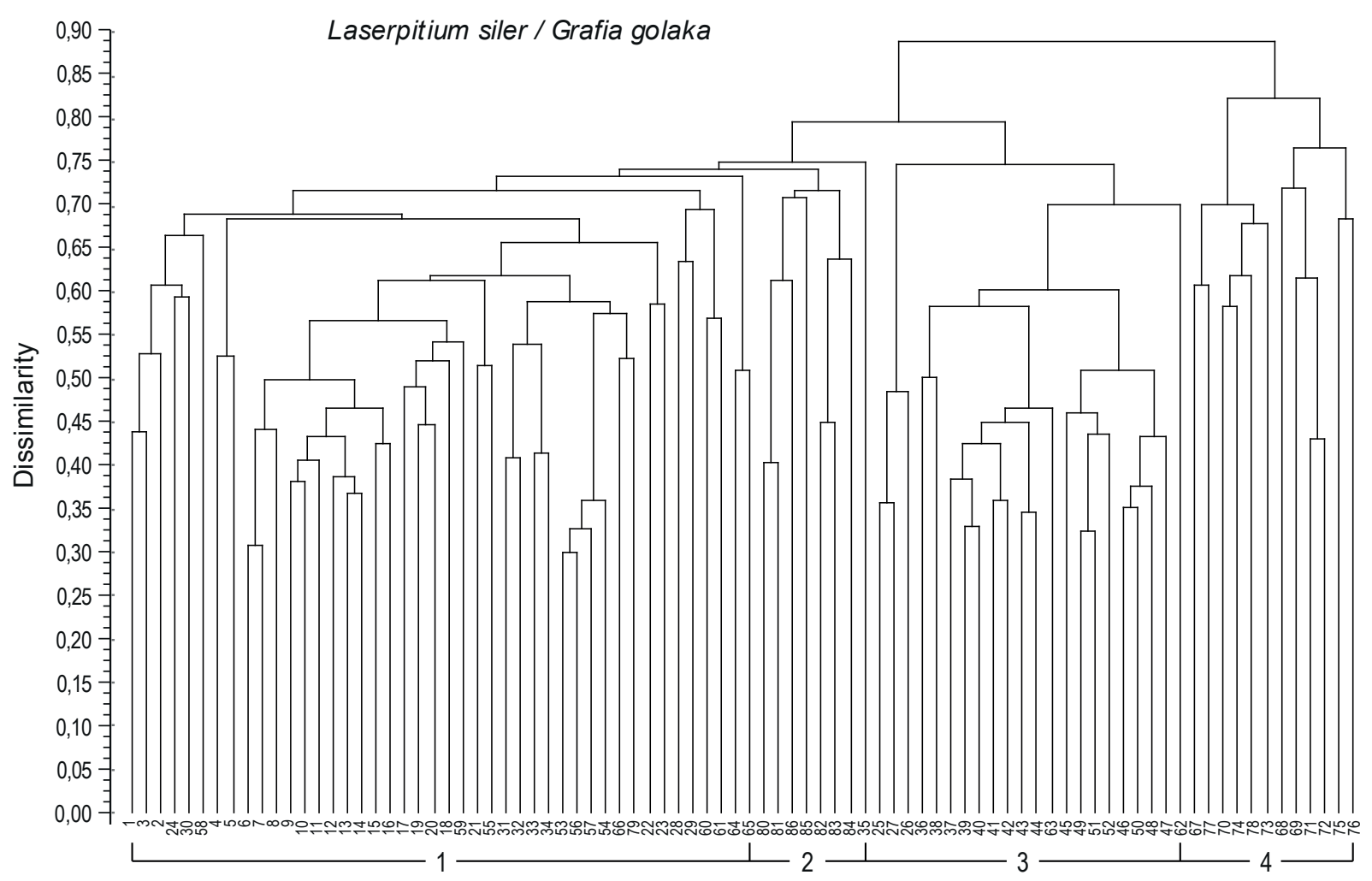

Figure 2: Dendrogram of tall herb communities with dominant Laserpitium siler or Grafia golaka in the Julian and Carnic Alps (NE Italy, W Slovenia) - UPGMA, similarity ratio (1 Centaureo julici-Laserpitietum sileris typicum, 2 Centaureo juliciLaserpitietum sileris var. geogr. Pedicularis elongata, 3 Centaureo julici-Laserpitietum sileris asphodeletosum albi, 4 Laserpitio sileri-Grafietum golakae).

Slika 2: Dendrogram združb visokih steblik z dominantnima vrstama Laserpitium siler ali Grafia golaka v Julijskih in Karnijskih Alpah (severovzhodna Italija, zahodna Slovenija) - UPGMA, similarity ratio (1 Centaureo julici-Laserpitietum sileris typicum, 2 Centaureo julici-Laserpitietum sileris var. geogr. Pedicularis elongata, 3 Centaureo julici-Laserpitietum sileris asphodeletosum albi, 4 Laserpitio sileri-Grafietum golakae).

Laserpitium siler/Grafia golaka

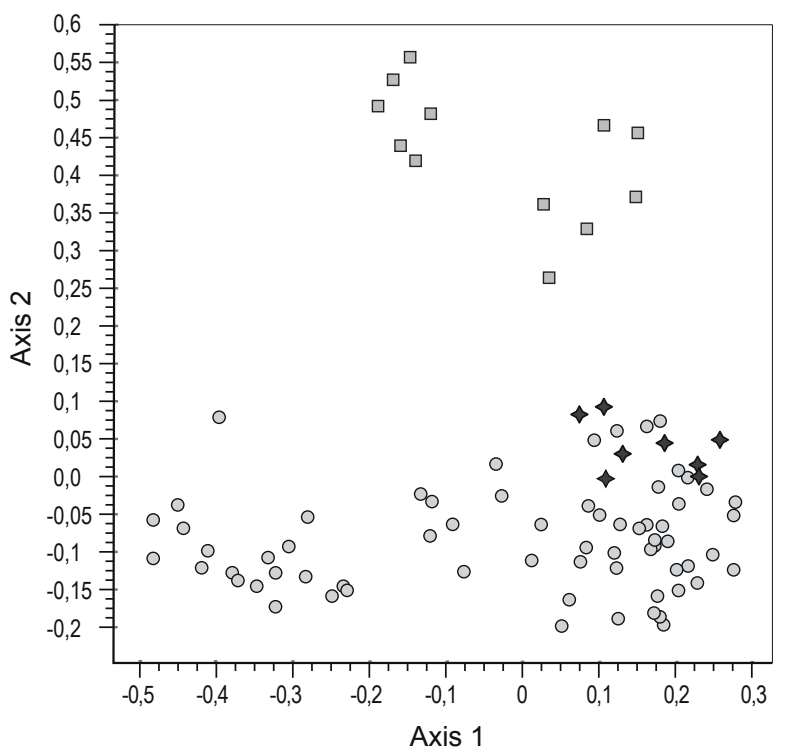

Figure 3: Two-dimensional scatter-diagram of tall herb communities with dominant Laserpitium siler or Grafia golaka in the Julian and Carnic Alps (NE Italy and W Slovenia) PCoA, similarity ratio ( $\mathrm{-}$ - Centaureo julici-Laserpitietum sileris - W Slovenia, $\diamond$-Centaureo julici-Laserpitietum sileris - NE Italy, $\square$-Laserpitio sileri-Grafietum golakae).

Slika 3: Dvorazsežni ordinacijski diagram združb visokih steblik z dominantnima vrstama Laserpitium siler ali Grafia golaka v Julijskih in Karnijskih Alpah (severovzhodna Italija, zahodna Slovenija) - PCoA, similarity ratio ( $\bigcirc$ - Centaureo julici-Laserpitetum sileris - zahodna Slovenija, $\diamond-$ Centaureo julici-Laserpitietum sileris - severovzhodna Italija, $\square-$ Laserpitio sileri-Grafietum golakae). 
Buphthalmum salicifolium, Koeleria pyramidata s. lat., Cirsium erisithales, Carlina acaulis s. lat. and Centaurea triumfettii. Differential species of the first main cluster are Bromopsis transsilvanica, Laserpitium peucedanoides and Festuca calva. Differential species of the second main cluster are Scabiosa hladnikiana, Tephroseris longifolia, Prunella vulgaris, Mercurialis ovata, Betonica officinalis, Salvia pratensis and Euphorbia verrucosa. Differential species of the first four syntaxa (Centaureo julici-Laserpitetum sileris s. lat.) are Gentiana lutea subsp. symphyandra, Serratula tinctoria subsp. macrocephala, Prunella grandiflora, for the typical geographical variant also Arabis pauciflora. Comparison of the syntaxa from the synthetic table (Table 5) clearly demonstrates that these are different communities (Fig-

\section{Laserpitium siler/Grafia golaka}

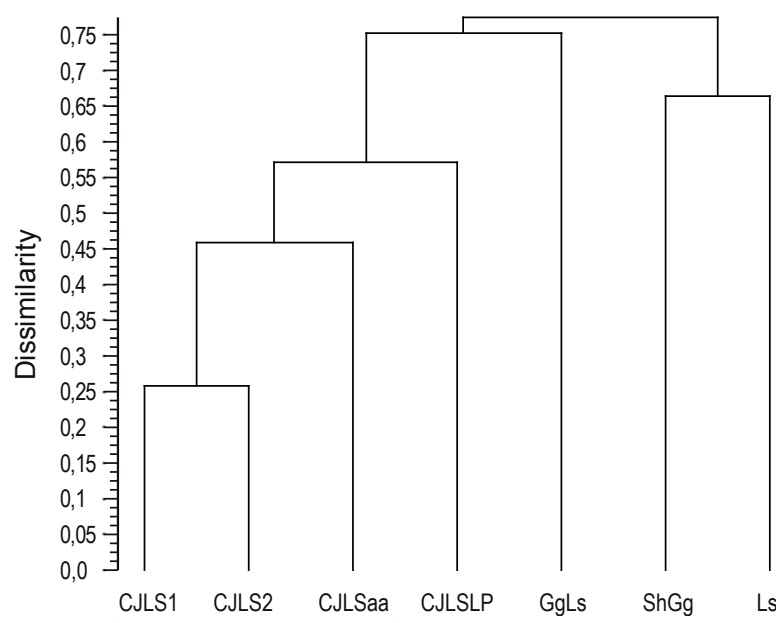

Figure 4: Dendrogram of tall herb and fringe communities with dominant Laserpitium siler or Grafia golaka in NE Italy and Slovenia - UPGMA, similarity ratio.

Slika 4: Dendrogram združb visokih steblik in gozdnih robov z dominantnima vrstama Laserpitium siler ali Grafia golaka v severovzhodni Italiji in Sloveniji - UPGMA, similarity ratio. ures 4 and 5). Stands of the association Centaureo julici-Laserpitietum sileris are distinctly different from the stands of the association Laserpitietum sileris and the stands of the association Laserpitio sileri-Grafietum golakae differ from the stands of the association Scabioso hladnikianae-Grafietum golakae. The differences are due to a different altitudinal belt and different potential natural vegetation. In terms of the association Laserpitietum sileris on the Gorjanci hills potential natural vegetation consists of submontane-montane beech forests from the associations Hacquetio-Fagetum, AruncoFagetum and Cardamino savensi-Fagetum; the stands of the association Scabioso hladnikiane-Grafietum golakae occur on the fringes of forest communities from the associations Ostryo-Fagetum, Arunco-

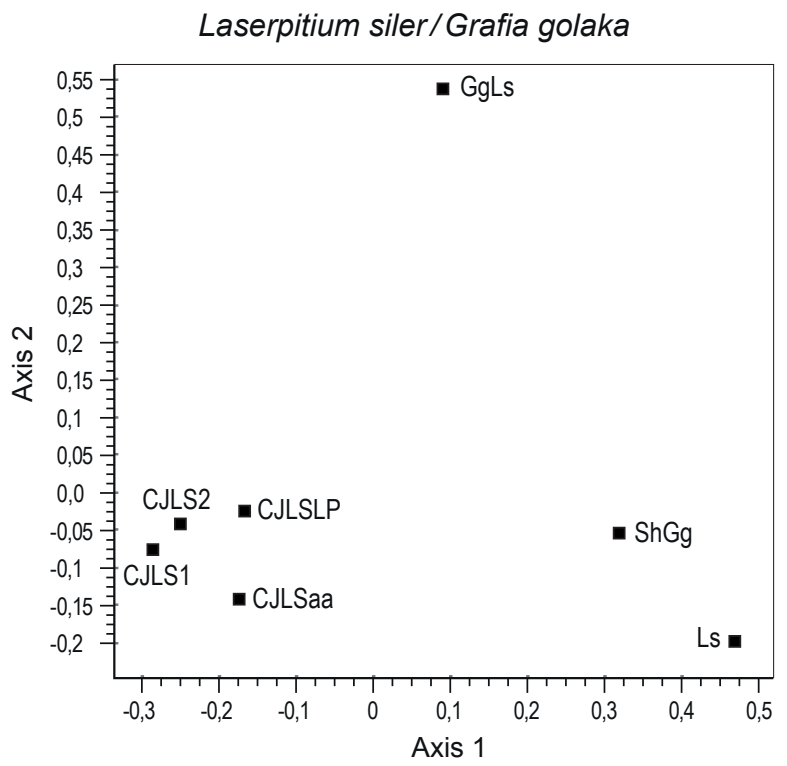

Figure 5: Two-dimensional scatter-diagram of tall herb and fringe communities with dominant Laserpitium siler or Grafia golaka in NE Italy and Slovenia - PCoA, similarity ratio.

Slika 5: Dvorazsežni ordinacijski diagram združb visokih steblik in gozdnih robov $\mathrm{z}$ dominantnima vrstama Laserpitium siler ali Grafia golaka v severovzhodni Italiji in Sloveniji - PCoA, similarity ratio.

\section{Legend to Figure 4 and 5}

CJLS1 Centaureo julici-Laserpitietum sileris ass. nov. hoc loco typicum - the Julian Alps, Slovenia

CJLS2 Centaureo julici-Laserpitietum sileris ass. nov. hoc loco typicum var. Brachypodium rupestre - the Julian Alps, Slovenia

CJLSaa Centaureo julici-Laserpitietum sileris ass. nov. hoc loco asphodeletosum albi subss. nov. - the Julian Alps, Slovenia (Dakskobler et al. 2007, table 1, rel. 8-33)

CJLSLP Centaureo julici-Laserpitietum sileris ass. nov. hoc loco var. geogr. Pedicularis elongata - the Carnic and W Julian Alps, NE Italy

GgLs Laserpitio sileri-Grafietum golakae ass. nov. hoc loco - the Julian Prealps, NE Italy

ShGg Scabioso hladnikianae-Grafietum golakae Čarni 2007 - central Slovenia (Čarni 2007, Table 1)

LS Laserpitietum sileris Springer 1987 var. geogr. Dianthus croaticus - southeastern Slovenia (Čarni et al. 2005, Tab. 1, rel. 47-56) 
Fagetum and Querco-Ostryetum; contact meadow communities (Scabioso hladnikianae-Caricetum humilis) are classified into the alliance Bromion erecti. Significant differences are demonstrated also by the comparison of the composition according to phytosociological groups (groups of diagnostic species) - Table 6. Stands of the typical form of the association Centaureo julici-Laserpitietum sileris are dominated by the species of subalpine-alpine grasslands and swards (Caricion austroalpinae, Elyno-Seslerietea), followed by the species from classes Festuco-Brometea and Trifolio-Geranietea. Stands of the subassociation Centaureo julici-Laserpitietum sileris asphodeletosum albi already have a predominant proportion of the species of classes FestucoBrometea and Trifolio-Geranietea. Subalpine stands with the dominant Laserpitium siler from the Carnic Alps and the western Julian Alps are very similar to other stands of the association Centaureo julici-Laserpitietum sileris from the southern and southwestern Julian Alps (see Figures 2, 3, 4 and 5); however, Centaurea haynaldii subsp. julica was not recorded there. These stands have a relatively large proportion of Erico-Pinetea species, indicating dolomite bedrock. The dominating species in the stands of the association Laserpitio sileri-Grafietum golakae are those of the class Festuco-Brometea, followed by the species of the classes MulgedioAconitetea and Trifolio-Geranietea. Stands of the associations Scabioso-Grafietum and Laserpitietum sileris are clearly dominated by the species of the class Festuco-Brometea, followed by those from the class Trifolio-Geranietea. There is also a large proportion of the species of the class Erico-Pinetea, at least in the stands of the association ScabiosoGrafietum. Čarni $(2005,2007)$ classifies the associations Scabioso-Grafietum and Laserpitietum sileris into the class Trifolio-Geranietea, order Origanetalia vulgaris, alliance Geranion sanguinei and suballiance Geranienion sanguinei. In our opinion, the proportion and abundance of diagnostic species of subalpine grasslands in the stands of the association Centaureo julici-Laserpitietum sileris is still considerable enough for this association to be classified into the alliance Caricion austroalpinae and class Elyno-Seslerietea. Preserved grasslands that are still mown are rare in this altitudinal belt and their floristic composition features relatively equal proportions of species from the classes Elyno-Seslerietea and Festuco-Brometea. This classification is somewhat questionable only for the stands in the lower altitudinal belt of their distribution, at 1100 to 1300 m a.s.l. (subassociation Centaureo
julici-Laserpitietum sileris asphodeletosum albi); alternatively, they could also be classified into the class Trifolio-Geranietea. In our opinion, classification of the association Laserpitio sileri-Grafietum golakae into higher syntaxonomic units should be subject to the consideration of phytosociological affinity of Grafia golaka. According to Poldini (1991: 399) Grafia golaka is a species of montane pastures, while Aeschimann et al. (2004 a: 1104) consider it a diagnostic species of the alliance Calamagrostion arundinaceae Oberdorfer 1950, i.e. a character species of thermo-hygrophilous montane-subalpine tall herb communities of the class Mulgedio-Aconitetea. Wraber (1990: 93) mentions its frequent occurrence on secondary montane pastures under the ridge of Breginjski Stol and in forest fringe communities in the belt of black hornbeam and flowering ash, as well as in the beech belt. Under the Stol and Muzci ridges Grafia golaka often thrives also in subalpine beech stands from the association Polysticho lonchitis-Fagetum. On its classic locality under Golaki Grafia golaka grows on very stony sites in natural clearings and in shrub stands with dominant Salix appendiculata and Rhododendron hirsutum (Rhododendro hirsuti-Salicetum appendiculatae nom. prov.) in the belt of subalpine beech forest (Polysticho lonchitis-Fagetum) - Table 7. Grafia golaka is therefore diagnostic for the tall herb communities from the class Mulgedio-Aconitetea and for the forest fringe communities from the class Trifolio-Geranietea. In our case, Laserpitio sileri-Grafietum golakae is a montane-subalpine community, so a more appropriate classification would be into the class Mulgedio-Aconitetea and into the alliance Calamagrostion arundinaceae.

Systematic classification and typification of the described new syntaxa is the following:

Elyno-Seslerietea Br.-Bl. 1948

Seslerietalia albicantis Br.-Bl. in Br.-Bl. \& Jenny 1926 corr. Oberd. 1983

Caricion austroalpinae Sutter 1962

Centaureo julici-Laserpitietum sileris Dakskobler in Dakskobler \& Poldini ass. nov. hoc loco

The nomenclatural type, holotypus, is relevé No. 13 in Table 1.

Diagnostic species of the association: Laserpitium siler, L. latifolium (dominant 
species of the community), Serratula tinctoria subsp. macrocephala $(=S$. tinctoria subsp. monticola), Lilium carniolicum, Gentiana lutea subsp. symphiandra, Carduus crassifolius, Centaurea haynaldii subsp. julica $(=C$. jacea subsp. julica $=$ C. . julica $)$ and Laserpitium peucedanoides (with their mainly eastern-Alpine or southeasternAlpine-Illyrian distribution the last five species characterize the new association also phytogeographically).

Centaureo julici-Laserpitietum sileris typicum

The nomenclatural type, holotypus, is relevé No. 13 in Table 1.

Centaureo julici-Laserpitietum sileris typicum var. Brachypodium rupestre var. prov.

Centaureo julici-Laserpitietum sileris asphodeletosum albi subass. nov. hoc loco

The nomenclatural type, holotypus, is relevé No. 15 in Table 1 (Dakskobler et al. 2007: 172-178).

The differential species is Asphodelus albus.

Centaureo julici-Laserpitietum sileris var. geogr. Pedicularis elongata

Differential species of the geographical variant are Pedicularis elongata, Knautia ressmannii and Euphorbia triflora subsp. kerneri.

Mulgedio-Aconitetea Hadač \& Klika in Klika \& Hadač 1944

Calamagrostietalia villosae Pawłovski in Pawłovski, Sokolowski et Wallisch 1928 (sin. Adenostyletalia alliariae G. \& J. Br.-Bl. 1931)

\section{Calamagrostion arundinaceae Oberdorfer 1950}

Laserpitio sileri-Grafietum golakae Poldini in Dakskobler \& Poldini ass. nov. hoc loco.

The nomenclatural type, holotypus, is relevé No. 9 in Table 4.

Diagnostic species: Grafia golaka, Laserpitium siler (dominant species; the first with its southeastern-European montane distribution characterizes the association also phytogeographically), Molopospermum peloponnesiacum subsp. bauhinii and Laserpitium peucedanoides (geographical differential species).

\section{CONCLUSIONS}

The steep, sunny slopes of the southern and southwestern Julian Alps on the natural timberline are mainly dominated by beech. In the past, subalpine beech stands were often cleared for hay meadows. These have been abandoned for more than half a century and are gradually becoming overgrown with spruce, in places also with Austrian (black) pine, hazel and other deciduous trees. Abandoned hay meadows are dominated by tall herbs, especially Laserpitium siler and L. latifolium, in some localities in the Julian Prealps (the Stol ridge / Gran Monte, the Muzci ridge / Cime del Monte Musi) also by Grafia golaka. Considerable allelopathic potential has been determined for these species and this, in addition to other factors such as snowslides, slows down the succession process. Relatively longterm stages are physiognomically distinct; they can be clearly spatially delimited and should preferably be treated at the syntaxonomic level of association. We therefore described two new associations, Centaureo julici-Laserpitietum sileris (presented in more detail but without a valid description some years ago - Dakskobler 2003, Dakskobler et al. 2007) and Laserpitio sileri-Grafietum golakae. Despite some similarities they are two syndynamically different communities. In terms of succession, the first is becoming gradually overgrown by pioneer subalpine spruce stands (Adenostylo glabrae-Piceetum sensu Zupančič = Homogyno sylvestris-Piceetum sensu Exner - see Dakskobler 2003), while spontaneous afforestation in the second community proceeds through shrubs Rubus idaeus, Salix appendiculata and deciduous tree species (Sorbus aria, S. aucuparia, Acer pseudoplatanus, in places also Ostrya carpinifolia, Amelanchier ovalis), and only rarely through Picea abies or Pinus nigra. The newly described associations therefore belong to different classes, the first to the class of subalpine-alpine grasslands, and the second to the class of montane and subalpine tall herb communities. The stands of both, but especially those of the first association, Centaureo julici-Laserpitietum sileris, are characterised by a species-rich composition and a diverse mixture of diagnostic species of montane and subalpine grasslands, forest fringes and tall herbs. They are a site of some rare and protected plants, such as Eryngium alpinum, Asphodelus albus and Iris sibirica subsp. erirrhiza, an important habitat type for some birds, e.g. corncrake (Crex crex), and in some areas (e.g. on the slopes of the Stol ridge) also a habitat type of European conservation concern. 


\section{POVZETEK}

Dve novi združbi visokih steblik $\mathrm{z}$ dominantnima vrstama Laserpitium siler in Grafia golaka v Jugovzhodnih Alpah (severovzhodna Italija, zahodna Slovenije)

Pred nekaj leti (Dakskobler 2003) smo preučevali razvoj gozda na nekdanjih senožetih v južnih Julijskih Alpah. Te senožeti so bile izkrčene v pasu subalpinskega bukovega gozda in smo jih kot razmeroma dolgotrajen sukcesijski stadij opisali kot provizorno novo asociacijo Centaureo julici-Laserpitietum sileris nom. prov. Kasnejše raziskave v različnih delih Julijskih Alp (zgornja Baška dolina, greben Breginjskega Stola, dolini Bale in Loške Koritnice) so pokazale, da je bila naša odločitev za samostojno asociacijo upravičena, saj ti sestoji oblikujejo fiziognomsko (po zunanjem videzu) in floristično jasno prepoznavno združbo, ki jo je moč dobro prostorsko omejiti in ki je zaradi počasnega zaraščanja razmeroma dolgotrajna. Novejše raziskave (prim. Kaligarič et al. 2011) kažejo na močan alelopatski potencial vrste Laserpitium siler, kar poleg drugih dejavnikov (snežni plazovi) zavira sukcesijski razvoj nazaj v gozd. Naredili smo precej dodatnih popisov in jim pridružili popise nekoliko podobnih sestojev v italijanskem delu Julijskih Alp in deloma tudi v Karnijskih Alpah. Primerjava zbranega popisnega gradiva je omogočila tipizacijo dveh novih asociacije, Centaureo julici-Laserpitietum sileris in Laserpitio sileri-Grafietum golakae.

Fitocenološke popise smo naredili po standardni srednjeevropski fitocenološki metodi (Braun-Blanquet 1964). Zbrane popise smo vnesli v bazo podatkov FloVegSi (Seliškar et al. 1993) in jih numerično obdelali s programskim paketom SYN-TAX 2000 (Podani 2001). V prvem koraki smo med seboj primerjali 87 popisov sestojev z dominantnima vrstama Laserpitium siler in Grafia gola$k a \mathrm{v}$ Julijskih Alpah, vključno s prigorjem (t. i. Julijske Predalpe), in Karnijskih Alpah - sliki 2 in 3. Sestoji z dominantno vrsto Grafia golaka iz Julijskih Predalp so se jasno razlikovali od sestojev $\mathrm{z}$ dominantno vrsto Laserpitium siler iz Julijskih in Karnijskih Alp. Na podlagi tega smo izdelali štiri analitske tabele (tabele 1, 2, 3, 4). V prvi so tipični sestoji asociacije Centaureo julici-Laserpitietum sileris, v tabeli 2 robni sestoji te asocicicije, ki kažejo na mogoče prehode $\mathrm{k}$ podobnim združbam visokih steblik in gorskih travišč (začasno jih vrednotimo kot varianto Centaureo julici-Laserpitietum sileris typicum var. Brachypodium rupestre), v tretji tabeli so sestoji z dominantno vrsto Laserpitium siler iz severovzhodne Italije (Centaureo julici-Laserpitietum sileris var. geogr. Pedicularis elongata) in v četrti tabeli sestoji z dominantno vrsto Grafia golaka (Laserpitio sileri-Grafietum golakae).

V drugem koraku smo izdelali sintezno tabelo (tabela 5), v katero smo poleg zgornjih štirih sintaksonov uvrstili še popise subasociacije Centaureo julici-Laserpitietum sileris asphodeletosum albi nom. prov. (Dakskobler et al. 2007, tab. 1, popisi 8-33) in dve podobni robni združbi iz osrednje in jugovzhodne Slovenije: Scabioso hladnikianae-Grafietum golakae Čarni 2007 iz Polhograjskega hribovja (Čarni 2007) in Laserpitietum sileris Springer 1987 var. geogr. Dianthus croaticus z Gorjancev (Čarni et al. 2005).

Primerjava sintaksonov iz sintezne tabele (tabela 5) jasno kažejo, da gre za različne združbe (sliki 4 in 5). Sestoji asociacije Centaureo julici-Laserpitietum sileris se očitno razlikujejo od sestojev asociacije Laserpitietum sileris in sestoji asociacije Laserpitio sileri-Grafietum golakae od sestojev asociacije Scabioso hladnikianae-Grafietum golakae. Razlike temeljijo na drugačnem višinskem pasu in drugačni potencialno naravni vegetaciji. Očitne razlike kaže tudi primerjava sestave po fitocenoloških skupinah (skupinah diagnostičnih vrst) - Tabela 6. V sestojih nove asociacije Centaureo julici-Laserpitietum sileris (nomenklaturni tip, holotypus, je fit. popis št. $13 \mathrm{v}$ tabeli 1) je delež diagnostičnih vrst subalpinskih travišč še tolikšen, da je smiselna uvrstitev te asociacije v zvezo Caricion austroalpinae in v razred Elyno-Seslerietea. Za uvrstitev nove asociacije Laserpitio sileri-Grafietum golakae (nomenkaturni tip, holotypus, je popis št. $9 \mathrm{v}$ tabeli 4) $\mathrm{v}$ višje sintakonomske enote je odločilna presoja, za katere združbe je značilna Grafia golaka. Uspeva tako v združbah visokih steblik iz razreda Mulgedio-Aconitetea kot $\mathrm{v}$ združbah gozdnih robovih iz razreda Trifolio-Geranietea. V našem primeru je Laserpitio sileri-Grafietum golakae montansko-subalpinska združba, zato je bolj ustrezna uvrstitev v razred Mulgedio-Aconitetea in v zvezo Calamagrostion arundinaceae.

Za sestoje obeh novo opisanih asociacij, še posebej prve, Centaureo julici-Laserpitietum sileris, je značilna bogata vrstna sestava in pisana zmes diagnostičnih vrst montanskih in subalpinskih travišč ter gozdnih robov in visokih steblik. So življenjski prostor nekaterih redkih in zavarovanih rastlin, npr. alpske možine (Eryngium alpinum), zlatega korena Asphodelus albus in kojniške perunike (Iris sibirica subsp. erirrhiza), pomem- 
ben habitatni tip za nekatere ptice, npr. za kosca (Crex crex) in na nekaterih območjih (npr. pobočja Stolovega grebena) tudi evropsko varstveno pomemben habitatni tip.

\section{ACKNOWLEDGEMENTS}

In our field work we were assisted by the late Prof. Dr. Tone Wraber, Mag. Boško Cuušin and Dr. Jože Bavcon (relevés under Breginjski Stol), and Dr. Gino Gobbo (relevés under the Muzci ridge / Cime del Monte Musi). Associate professor Dr. Andraž Čarni helped with literature and his advice. Sincere thanks to two anonymous reviewers for their helpful remarks and improvements. Iztok Sajko made the synoptic picture of the study area and improved the Figure 2. English translation by Andreja Šalamon Verbič.

\section{REFERENCES}

Aeschimann, D., Lauber, K., Moser, D. M. \& Theurillat, J.-P. 2004 a: Flora alpina. Bd. 1: Lycopodiaceae-Apiaceae. Haupt Verlag, Bern, Stuttgart, Wien, 1159 pp.

Aeschimann, D., Lauber, K., Moser, D. M. \& Theurillat, J.-P. 2004 b: Flora alpina. Bd. 2: Gentianaceae-Orchidaceae. Haupt Verlag, Bern, Stuttgart, Wien, 1188 pp.

Aeschimann, D., Lauber, K., Moser, D. M. \& Theurillat, J.-P. 2004 c: Flora alpina. Bd. 3: Register. Haupt Verlag, Bern, Stuttgart, Wien, 322 pp.

Braun-Blanquet, J. 1964: Pflanzensoziologie. Grundzüge der Vegetationskunde. 3. Auflage. Springer, Wien - New York, 865 pp.

Čarni, A. 1997: Syntaxonomy of the Trifolio-Geranietea (Saum Vegetation) in Slovenia. Folia Geobotanica et Phytotaxonomica 32: 207-219.

Čarni, A. 2005: Trifolio-Geranietea vegetations in south and southeast Europe. Acta Botanica Gallica 152: 483-496.

Čarni, A. 2007: Vegetation of forest fringes on shallow dolomite bedrock in Central Slovenia. Collection of papers devoted to academician Kiril Micevski on the occasion of the 80 years of his bearth. Macedonian Academy of Science and Arts, Skopje, pp. 237-248.

Čarni, A., Franjić, J., Šilc, U., Škvorc, Ž. 2005: Floristical, ecological and structural diversity of vegetation of forest fringes of the Northern
Croatia along a climatic gradient. Phyton (Horn) 45 (2): 287-303.

Čušin, B. 2006: Rastlinstvo Breginjskega kota. Založba ZRC, ZRC SAZU, Ljubljana, 198 pp.

Dakskobler, I. 2003: Pioneer spruce stands above the actual (anthropogenic) upper forest line in the southern Julian Alps (an example from the upper Bača Valley). Hacquetia 2 (1): 19-37.

Dakskobler, I., Vreš, B. Anderle, B. 2007: Novosti v Flori slovenskega dela Julijskih Alp. Novelties of flora in the Slovenian part of the Julian Alps. Razprave 4. razreda SAZU 48 (2): 139-192.

Ewald, J. 1996: Graslahner - Rasengesellschaften in der montanen Waldstufe der Tegernseer Kalkalpen. Berichte der Bayerischen Botanischen Gesellschaft (München) 66/67: 115-133.

Gils van, H., Kaysers, E.\& Launspach, W. 1975: Saumgesellschaften im klimazonalen Bereich des Ostryo-Carpinion orientalis. Vegetatio 31 (1): 47-64.

Gobbo, G. \& Poldini, L. 2005: La diversità floristica del parco delle Prealpi Giulie. Atlante corologico. Università degli Studi di Trieste, Dipartimento di Biologia, Trieste, 364 pp.

Grabherr, G., Greimler, J. \& Mucina, L. 1993: Seslerietea albicantis. In: Grabherr, G. \& Mucina L.(eds.): Die Pflanzengesellschaften Österreichs. Teil II: Natürliche waldfreie Vegetation, Gustav Fischer Verlag, Jena - Stuttgart New York, pp. 402-446.

Jarvis A., Reuter, H. I., Nelson, A., Guevara, E. 2008: Hole-filled seamless SRTM data V4. International Centre for Tropical Agriculture (CIAT), available from http://srtm.csi.cgiar. org.

Kaligarič, M. 1997: Rastlinstvo Primorskega krasa in Slovenske Istre: travniki in pašniki. Zgodovinsko društvo za južno Primorsko, Znanstveno raziskovalno središče Republike Slovenije Koper (Annales majora), Koper, 111 pp.

Kaligarič, M. \& Poldini, L. 1997: Nuovi contributi per una tipologia fitosociologica delle praterie magre (Scorzoneretalia villosae $\mathrm{H}$-ić 1975) del Carso nordadriatico. Gortania 19: 119-148.

Kaligarič, M., Meister, M. H., Škornik, S., Šajna, N., Kramberger B. \& Bolhàr-Nordenkampf, H. R. 2011: Grassland succesion is mediated by umbelliferous colonizers showing allelopathic potential. Plant Biosystems 145 (3): 688-698. 
Maarel van der, E. 1979: Transformation of cover-abundance values in phytosociology and its effects on community similarity. Vegetatio 39 (2): 97-114.

Martinčič, A. 2003: Seznam listnatih mahov (Bryopsida) Slovenije. Hacquetia 2 (1): 91-166.

Martinčič, A., Wraber, T., Jogan, N., Podobnik, A., Turk, B., Vreš, B., Ravnik, V., Frajman, B., Strgulc Krajšek, S., Trčak, B., Bačič, T., Fischer, M. A., Eler, K. \& Surina, B. 2007: Mala flora Slovenije. Ključ za določanje praprotnic in semenk. Tehniška založba Slovenije, Ljubljana, 967 pp.

Oberdorfer, E. 1978: Carici rupestris-Kobresietea bellardii Ohba 74. In: Oberdorfer, E. (ed.): Süddeutsche Pflanzengesellschaften, Teil II, 2. Aufl., Gustav Fischer Verlag, Stuttgart New York, pp. 181-203.

Pedrotti, F. 1970: Tre nuove associazioni erbacee di substrati calcarei in Trentino. Studi Trentini di Scienze Naturali, Sezione Biologica (Trento) 47 (2): 252-263.

Podani, J. 2001: SYN-TAX 2000. Computer Programs for Data Analysis in Ecology and Systematics. User's Manual, Budapest, 53 pp.
Poldini, L. 1991: Atlante corologico delle piante vascolari nel Friuli-Venezia Giulia. Inventario floristico regionale. Regione Autonomo FriuliVenezia Giulia \& Università di Trieste, Udine, 898 pp.

Poldini, L. 2003: Einige neue Saumgesellschaften aus dem Voralpenraum Friauls (NO Italien). In: Čarni, A. \& Šuštar, B. (eds.): International symposium on vegetation in SE Europe. Otočec 2003. Abstract book, Otočec, pp. 7.

Seliškar, T., Vreš, B. \& Seliškar, A. 2003: FloVegSi 2.0. Računalniški program za urejanje in analizo bioloških podatkov. Biološki inštitut ZRC SAZU, Ljubljana.

Šilc, U. \& Čarni, A. 2012: Conspectus of vegetation syntaxa in Slovenia. Hacquetia 11(1): 113-164.

Wraber, T. 1990: Sto znamenitih rastlin na Slovenskem. Prešernova družba, Ljubljana, 239 pp. 


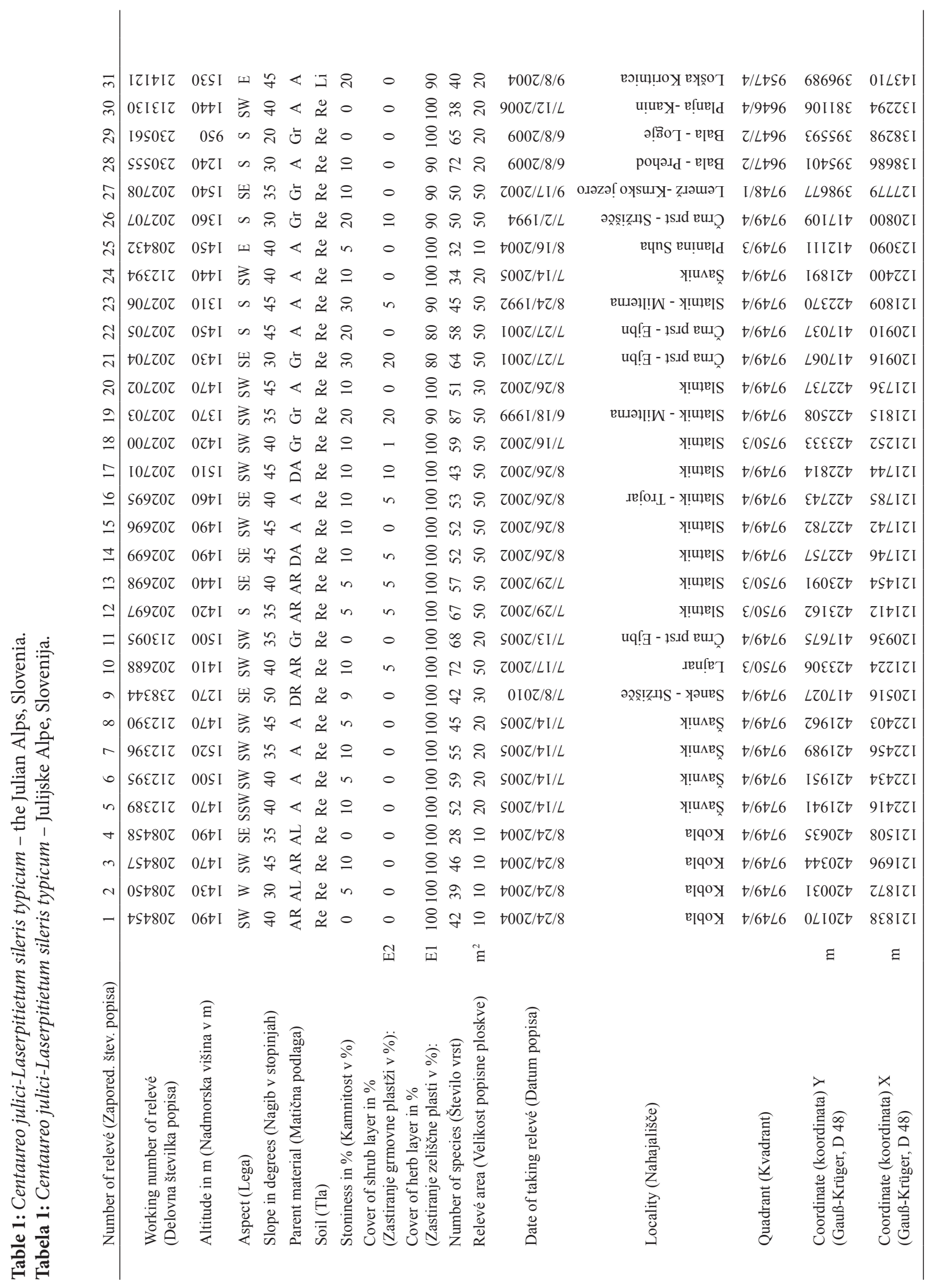




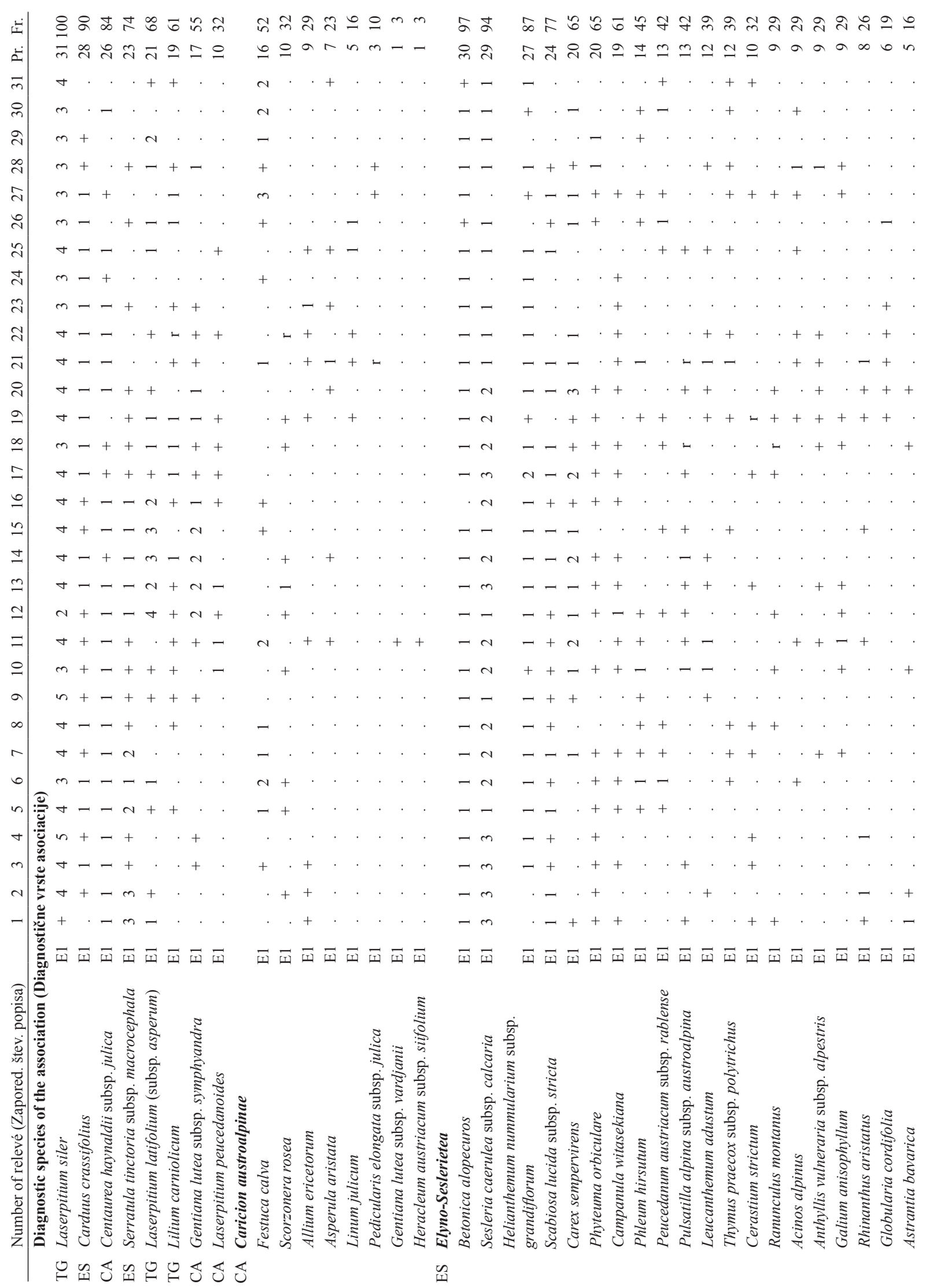




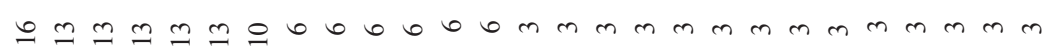

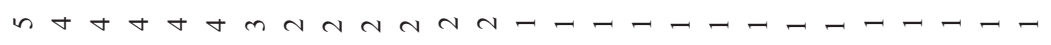
$+$

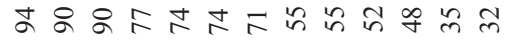
ते $+++++\cdot \cdot \cdot+\cdot+$.

N

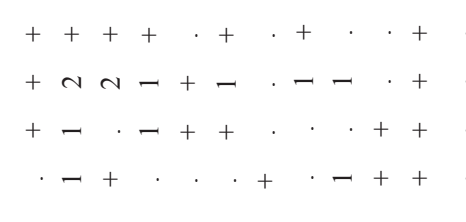$$
\begin{aligned}
& - \\
& +
\end{aligned}
$$

$$
\begin{aligned}
& +
\end{aligned}
$$

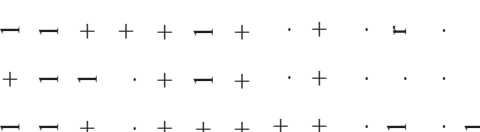$$
-
$$$$
-+++\cdots+-
$$$$
-++\cdot \cdot \cdot+\cdot++ \text {. }
$$$$
+-\mathrm{N}+++\cdot+\cdot+++
$$$$
++++++-++. \cdot \cdot+
$$$$
+-++-{ }_{+}+++\cdot
$$$$
+--+++-+++++
$$$$
--\longrightarrow+++-+++\cdots
$$$$
-++\mathrm{N}+++-\mathrm{N} \cdot+\text {. }
$$$$
-+\cdot-++-++\cdots
$$$$
+---+++\cdot++.
$$$$
+-\mathrm{N}+++\cdot \cdot \cdot+\cdot+
$$$$
+---++
$$$$
++--+\cdots+
$$$$
+--\neg++-\cdot \cdot \cdot \cdot+
$$$$
+\quad-
$$$$
+-+++\cdots++
$$$$
+-+-\neg++\cdot+-
$$

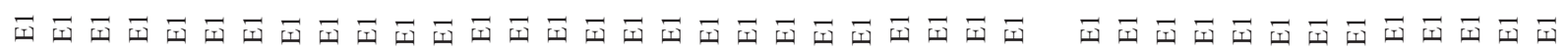

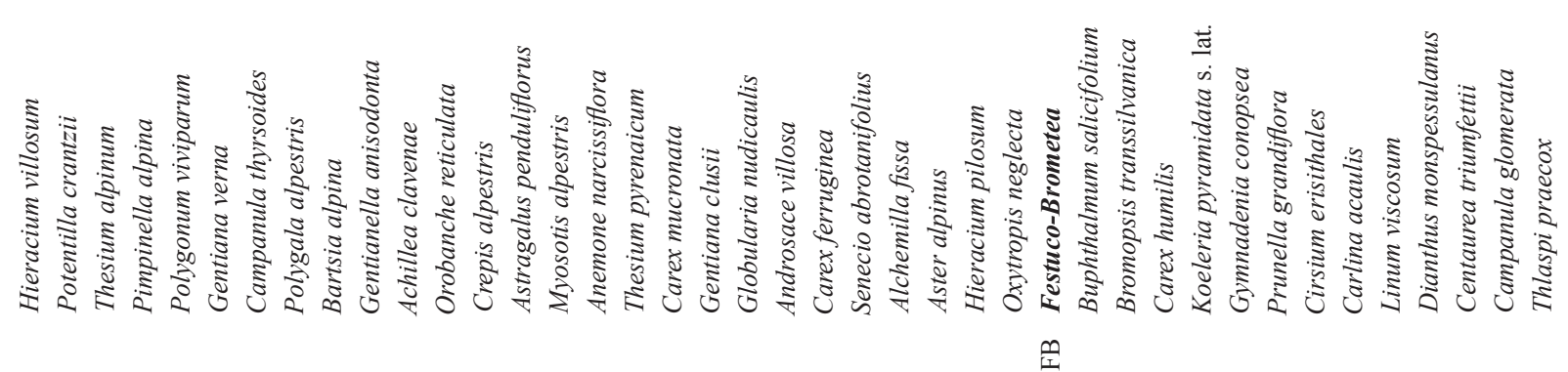




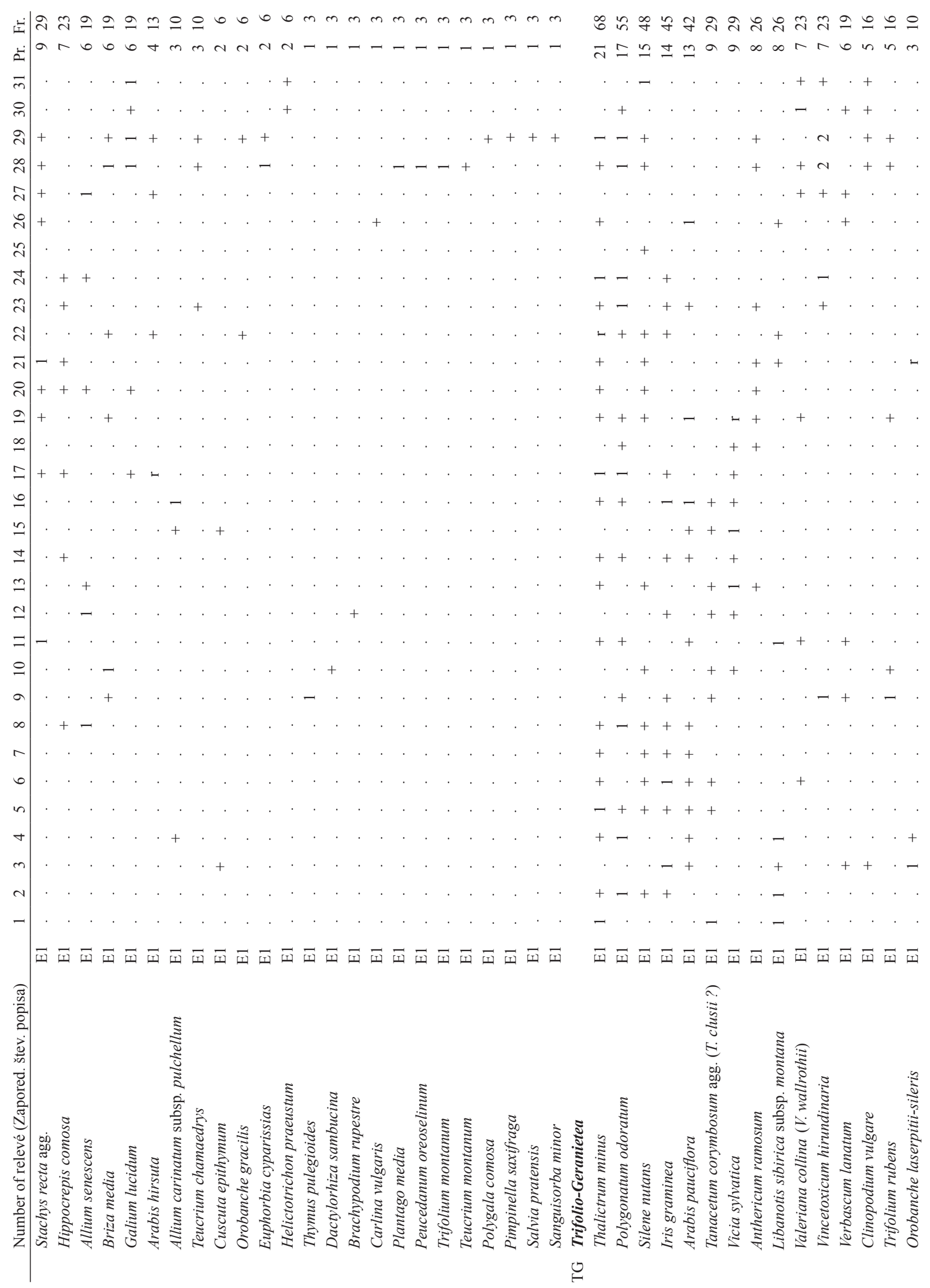




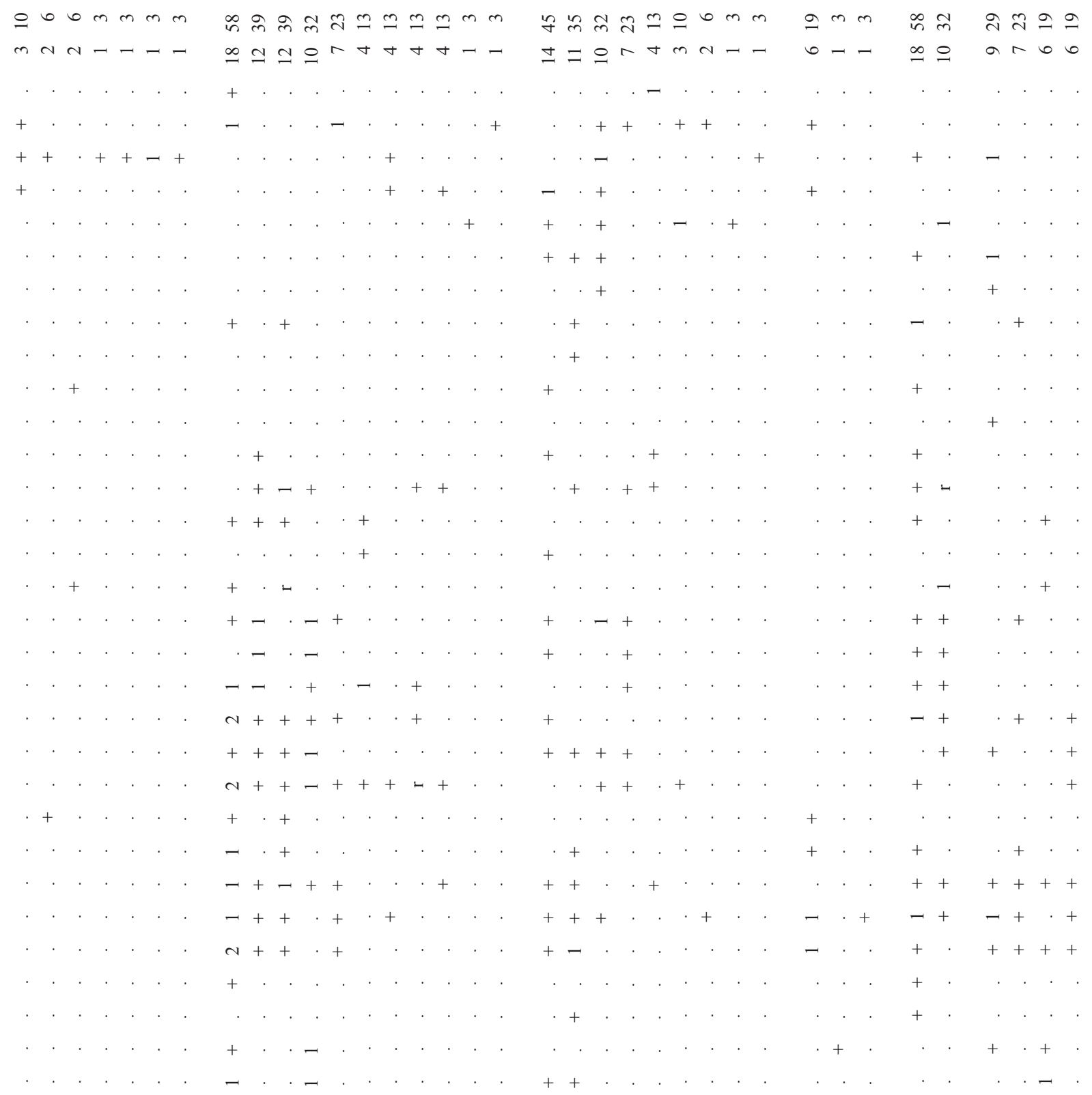

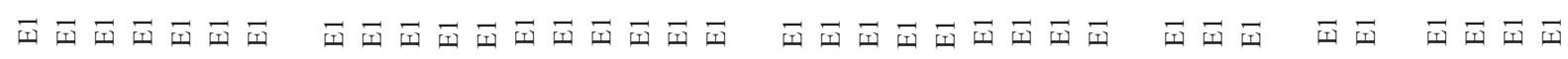

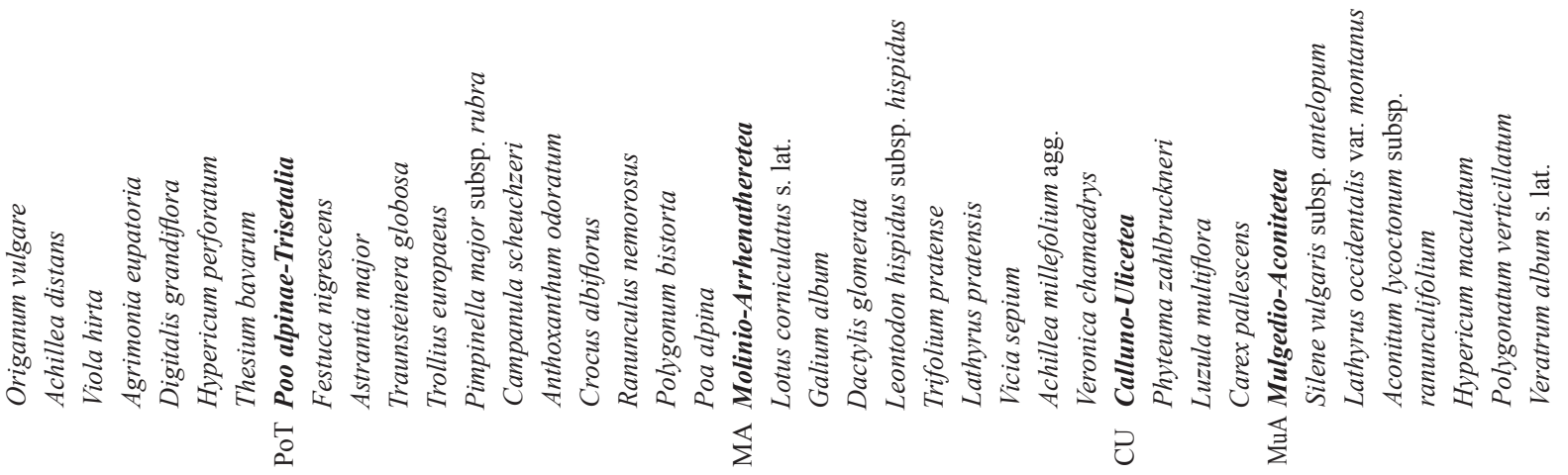




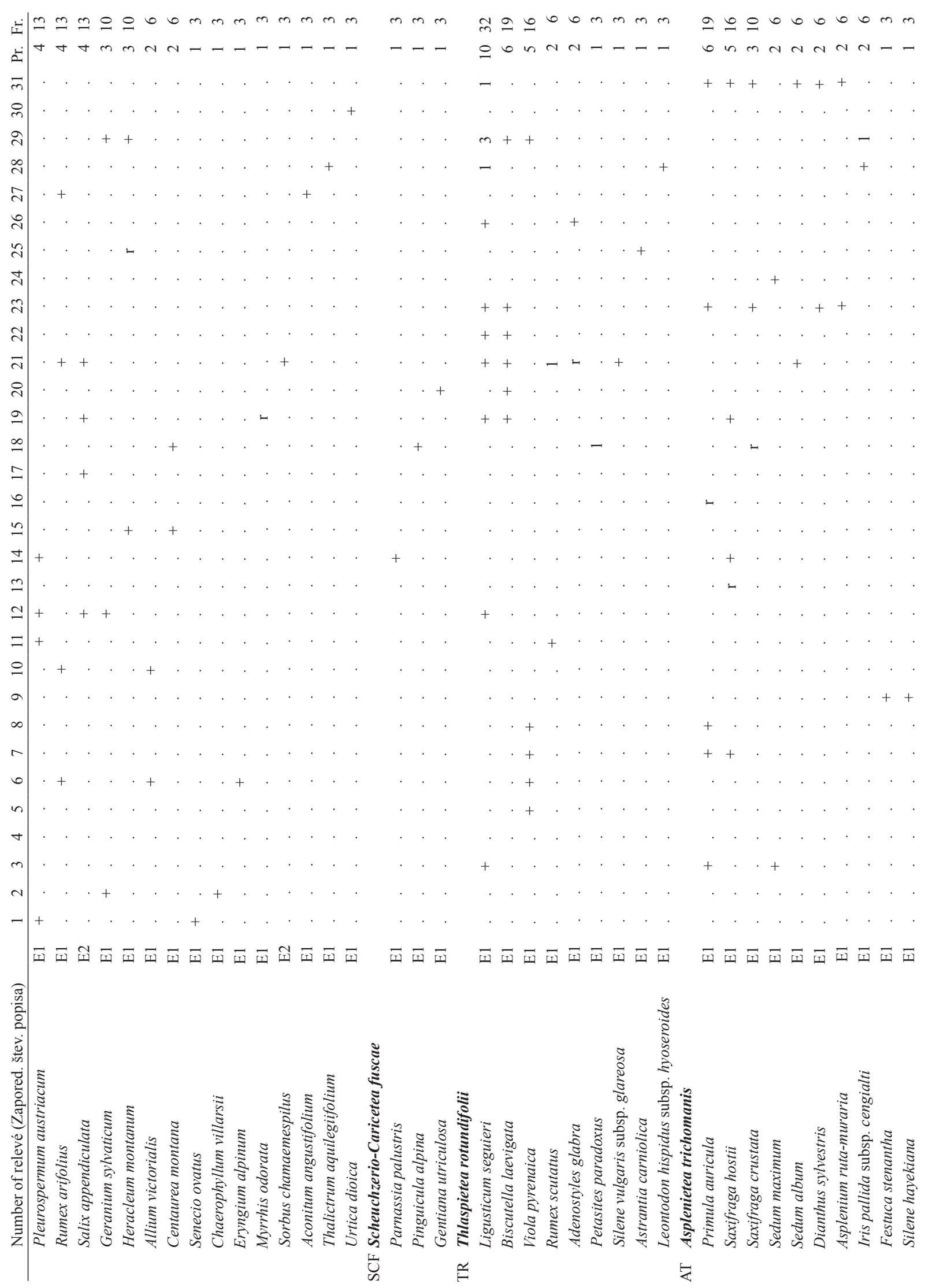




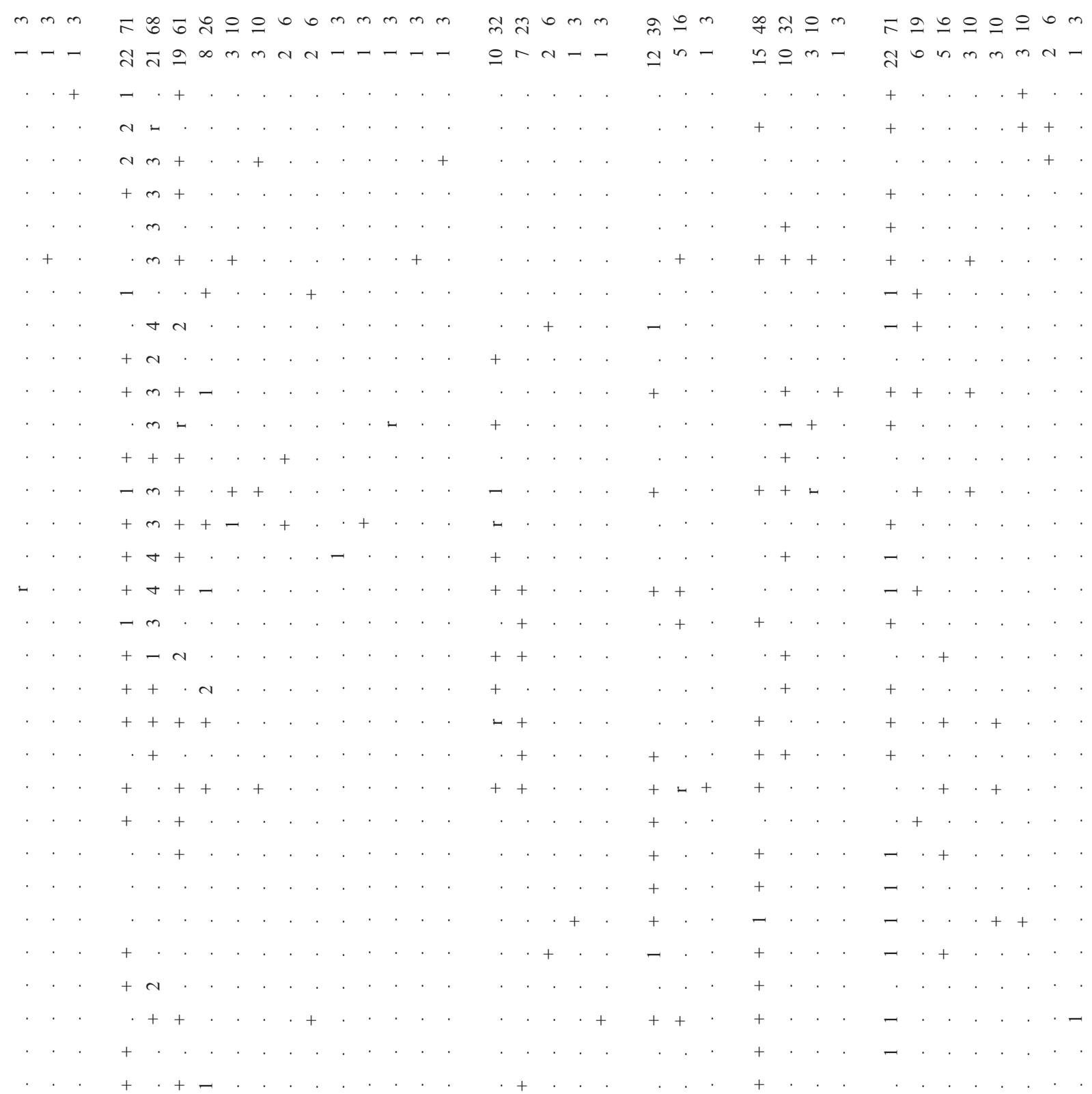

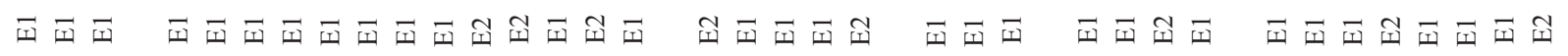

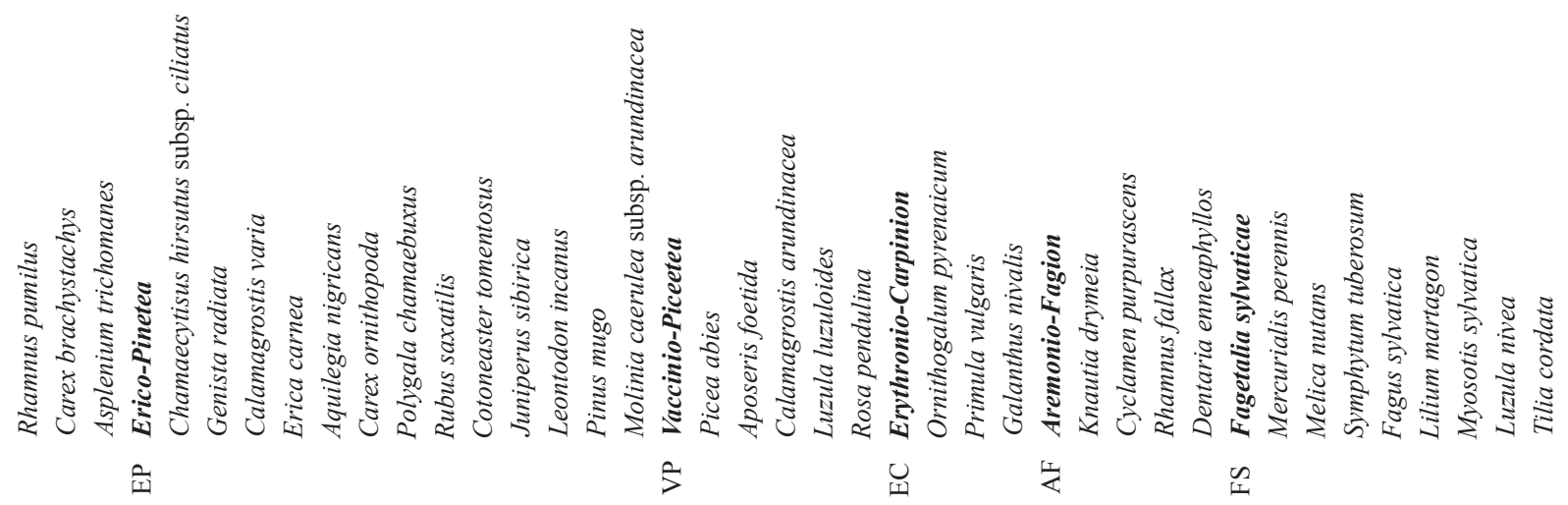




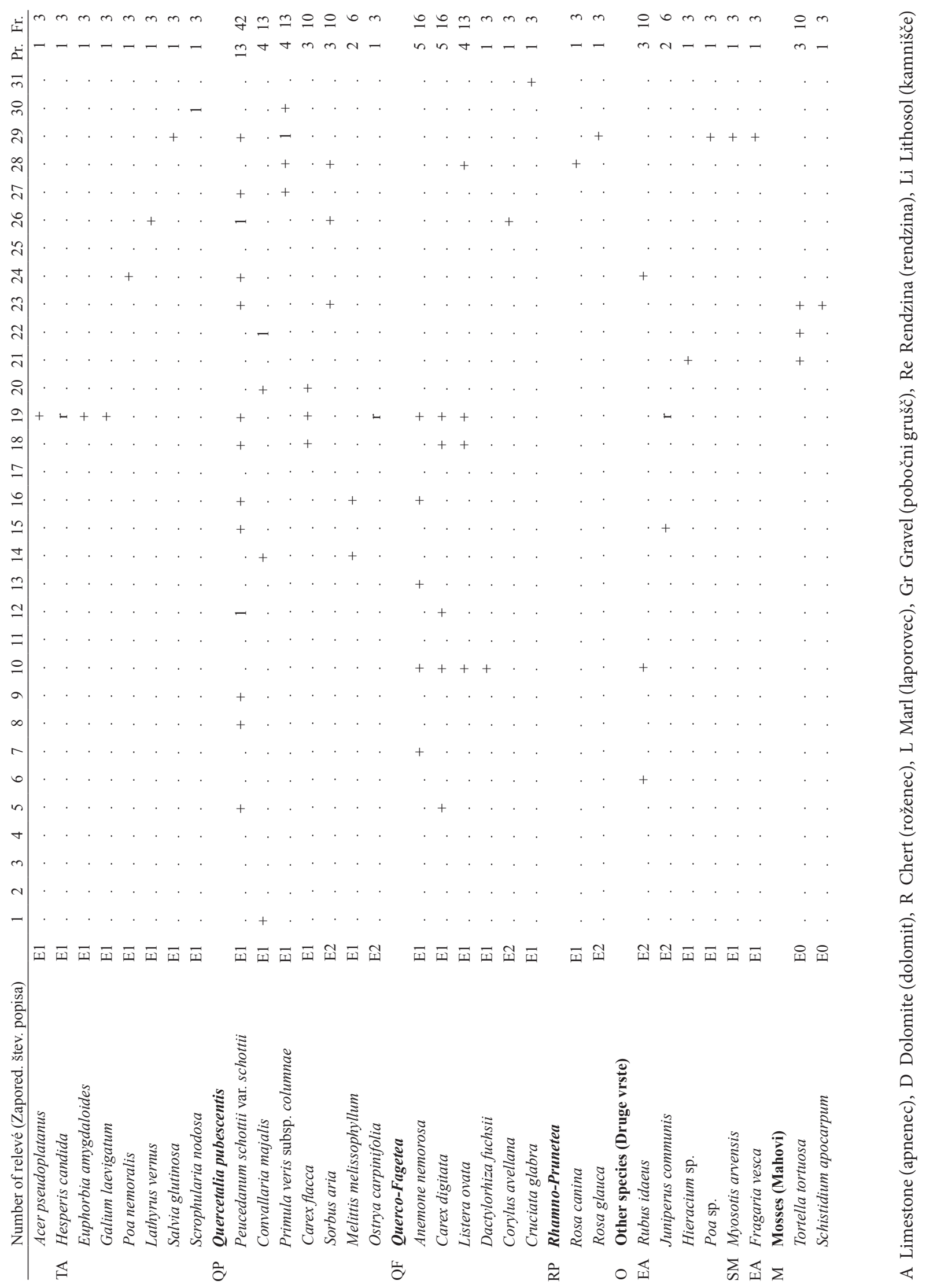


Table 2: Centaureo julici-Laserpitietum sileris typicum var. Brachypodium rupestre - the Julian Alps, Slovenia.

Table 2: Centaureo julici-Laserpitietum sileris typicum var. Brachypodium rupestre - Julijske Alpe, Slovenija.

Number of relevé

Working number of relevé

Altitude in $m$

Aspect

Slope in degrees

Parent material

Soil

Stoniness in \%

Cover of shrub layer in \%

Cover of herb layer in $\%$

Number of species

Relevé area

Date of taking relevé

Locality

Quadrant

Coordinate Y (Gauß-Krüger, D 48)

Coordinate X (Gauß-Krüger, D 48)

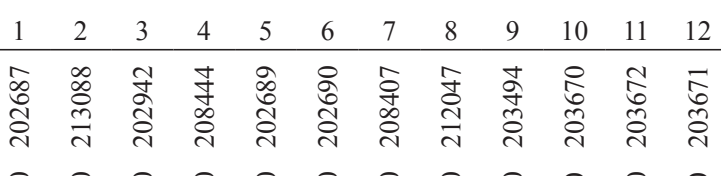

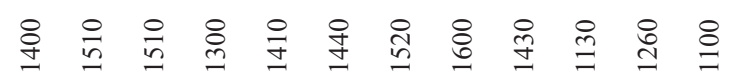

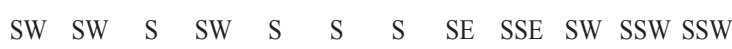

$\begin{array}{llllllllllll}40 & 30 & 35 & 40 & 35 & 40 & 35 & 10 & 30 & 30 & 30 & 25\end{array}$

AR AG A ALR ALR ALR A A AR A Gr Gr

$\begin{array}{llllllllllll}\mathrm{R} & \mathrm{E} & \mathrm{R} & \mathrm{R} & \mathrm{R} & \mathrm{R} & \mathrm{Li} & \mathrm{R} & \mathrm{R} & \mathrm{R} & \mathrm{R} & \mathrm{R}\end{array}$

$\begin{array}{llllllllllll}0 & 0 & 5 & 0 & 10 & 10 & 20 & 0 & 10 & 5 & 5 & 5\end{array}$

E2 $0 \begin{array}{llllllllllll}0 & 0 & 0 & 0 & 10 & 1 & 0 & 10 & 10 & 0 & 0 & 2\end{array}$

$\begin{array}{lllllllllllll}\text { E1 } & 100 & 100 & 100 & 100 & 100 & 90 & 100 & 90 & 90 & 100 & 100 & 100\end{array}$

$\begin{array}{llllllllllll}56 & 60 & 60 & 49 & 52 & 49 & 57 & 41 & 72 & 81 & 69 & 73\end{array}$

$\mathrm{m}^{2} \quad \begin{array}{llllllllllll}50 & 20 & 20 & 20 & 10 & 50 & 50 & 15 & 50 & 50 & 50 & 50\end{array}$

究

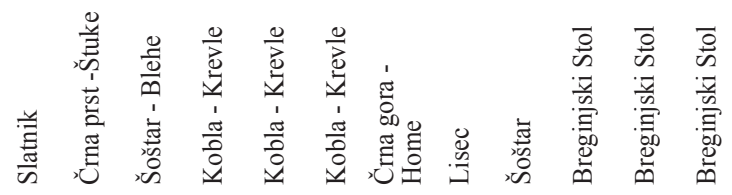

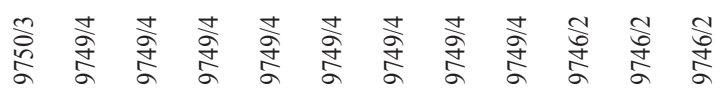

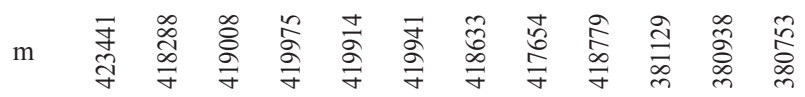

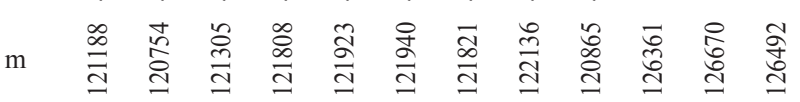

Diagnostic species of the association

CA Centaurea haynaldii subsp. julica

TG Laserpitium siler

ES Serratula tinctoria subsp. macrocephala

TG Laserpitium latifolium (subsp. asperum)

ES Carduus crassifolius

CA Laserpitium peucedanoides

TG Lilium carniolicum

CA Gentiana lutea subsp. symphyandra

CA Caricion austroalpinae

Festuca calva

Scorzonera rosea

Allium ericetorum

Asperula aristata

Linum julicum

Heracleum austriacum subsp. siifolium

Pedicularis elongata subsp. julica

\begin{tabular}{|c|c|c|c|c|c|c|c|c|c|c|c|c|c|}
\hline & & & & & & & & & & & & & Pr. Fr. \\
\hline E1 & 1 & 1 & 1 & 1 & + & + & + & + & + & 1 & + & + & $12 \quad 100$ \\
\hline E1 & + & & + & 2 & 1 & 2 & 3 & 3 & 1 & 1 & 1 & 2 & 11 \\
\hline E1 & 2 & 3 & 1 & 3 & 1 & 1 & & 1 & & 1 & + & + & 10 \\
\hline E1 & 2 & 2 & . & 1 & 1 & 2 & 1 & . & + & 3 & 2 & + & 10 \\
\hline E1 & + & . & + & . & . & . & + & + & . & 1 & 2 & 1 & 58 \\
\hline E1 & + & + & 1 & + & $\mathrm{r}$ & . & . & . & + & . & . & . & 50 \\
\hline E1 & . & . & + & . & . & . & + & . & . & 1 & + & + & 42 \\
\hline E1 & . & . & . & + & . & . & . & + & . & 1 & 2 & . & 33 \\
\hline
\end{tabular}

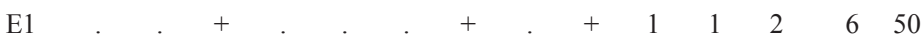

$\mathrm{E} 1++1+1 \quad 1 . \quad . \quad . \quad . \quad . \quad 650$

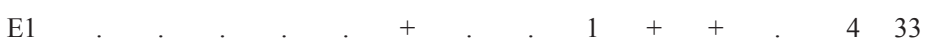

E1 . . . . . . . . $+{ }^{2}++433$

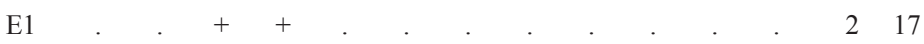

E1

E1

ES Elyno-Seslerietea

Betonica alopecuros

Phyteuma orbiculare

Helianthemum nummularium subsp. grandiflorum E1

Scabiosa lucida subsp. stricta

E1

$\mathrm{E} 111+1+\begin{array}{llllllllll}1 & 1 & 1 & 1 & 1 & 1 & 1 & 1 & 12 & 100\end{array}$

$\mathrm{E} 1+++++++.+2.975$ 
Number of relevé

Campanula witasekiana

Phleum hirsutum

Sesleria caerulea subsp. calcaria

Leucanthemum adustum

Pulsatilla alpina subsp. austroalpina

Carex sempervirens

Galium anisophyllum

Anthyllis vulneraria subsp. alpestris

Carex ferruginea

Hieracium villosum

Astragalus penduliflorus

Campanula thyrsoides

Peucedanum austriacum subsp. rablense

Anemone narcissiflora

Polygonum viviparum

Rhinanthus aristatus

Acinos alpinus

Bartsia alpina

Thymus praecox subsp. polytrichus

Astrantia bavarica

Crepis bocconi

Ranunculus montanus

Carduus defloratus

Thesium alpinum

Cerastium strictum

Pimpinella alpina

FB Festuco-Brometea

Bromopsis transsilvanica

Buphthalmum salicifolium

Carlina acaulis

Cirsium erisithales

Koeleria pyramidata (s. lat.)

Brachypodium rupestre

Dianthus monspessulanus

Campanula glomerata

Centaurea triumfettii

Gymnadenia conopsea

Prunella grandiflora

Briza media

Carex humilis

Linum viscosum

Iris sibirica subsp. erirrhiza

Trifolium montanum

Helictotrichon praeustum

Galium verum

Inula hirta

Allium carinatum subsp. carinatum

Cirsium pannonicum

Galium lucidum

Peucedanum oreoselinum

Plantago media

Veronica teucrium

Helianthemum nummularium subsp. obscurum

Thlaspi praecox

\begin{tabular}{|c|c|c|c|c|c|c|c|c|c|c|c|c|c|c|}
\hline & 1 & 2 & 3 & 4 & 5 & 6 & 7 & 8 & 9 & 10 & 11 & 12 & Pr. & Fr. \\
\hline E1 & + & + & + & & . & + & . & . & + & + & + & + & 8 & 67 \\
\hline E1 & + & . & + & . & + & . & . & + & + & 1 & 1 & 1 & 8 & 67 \\
\hline E1 & . & 1 & + & 1 & + & . & . & $\cdot$ & + & + & . & + & 7 & 58 \\
\hline E1 & . & . & + & ${ }^{\circ}$ & . & + & + & . & + & + & + & . & 6 & 50 \\
\hline E1 & + & + & 1 & . & . & + & + & . & . & . & . & . & 5 & 42 \\
\hline E1 & . & + & 1 & . & + & + & . & . & . & . & . & . & 4 & 33 \\
\hline E1 & . & + & + & + & . & . & ${ }^{\prime}$ & . & . & . & $\mathrm{r}$ & . & 4 & 33 \\
\hline E1 & . & . & + & . & . & . & + & . & . & + & + & . & 4 & 33 \\
\hline E1 & . & . & + & 2 & . & . & 1 & . & + & . & . & . & 4 & 33 \\
\hline E1 & . & . & + & . & . & $\mathrm{r}$ & + & . & . & + & . & . & 4 & 33 \\
\hline E1 & . & . & . & 2 & + & + & . & . & 1 & . & . & . & 4 & 33 \\
\hline E1 & . & . & . & . & . & . & . & . & + & + & + & + & 4 & 33 \\
\hline E1 & + & . & . & . & . & . & + & + & . & . & . & . & 3 & \\
\hline E1 & . & + & + & . & . & . & + & . & . & . & . & . & 3 & 25 \\
\hline E1 & . & . & + & + & + & . & . & . & . & . & . & . & 3 & 25 \\
\hline E1 & . & . & . & . & . & . & . & + & + & . & . & 2 & 3 & 25 \\
\hline E1 & . & + & . & . & . & . & . & . & . & . & . & + & 2 & 17 \\
\hline E1 & . & + & + & . & . & . & . & . & . & . & . & . & 2 & 17 \\
\hline E1 & . & . & + & . & . & . & . & . & 1 & . & . & . & 2 & 17 \\
\hline E1 & + & . & . & . & . & . & . & . & . & . & . & . & 1 & \\
\hline E1 & . & . & 1 & . & . & . & . & . & · & . & . & . & 1 & \\
\hline E1 & . & . & + & . & . & . & . & . & . & . & . & . & 1 & \\
\hline E1 & . & . & . & . & . & . & 1 & . & . & . & . & . & 1 & \\
\hline E1 & . & . & . & . & . & . & + & . & . & . & . & . & 1 & \\
\hline E1 & . & . & . & . & . & . & . & . & + & . & . & . & 1 & \\
\hline E1 & . & . & . & . & . & . & . & . & . & . & + & . & 1 & \\
\hline
\end{tabular}

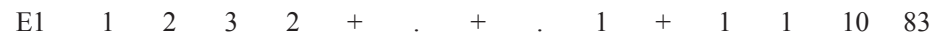

$\mathrm{E} 11++1.2 .1+1+{ }^{2}+101083$

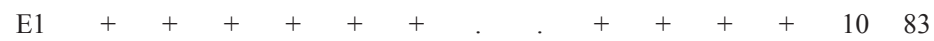

$\mathrm{E} 12+.1++1+2.975$

$\mathrm{E} 1.2+.+2 .+2122758$

$\mathrm{E} 12 .+2 . \quad . \quad 123+650$

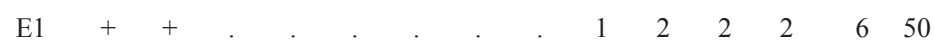

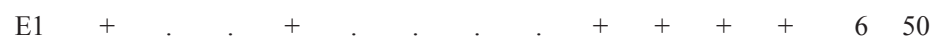

$\mathrm{E} 1 .++.+2 .+2 .+4650$

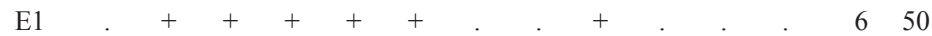

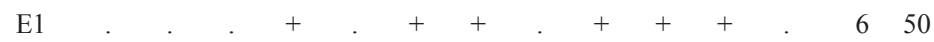

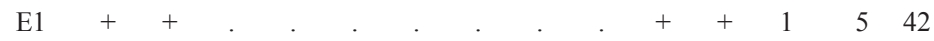

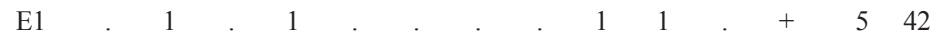

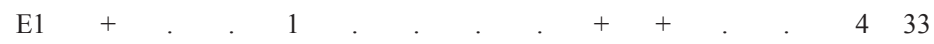

$\mathrm{E} 1 .+.+2 .+2 . \quad . \quad 325$

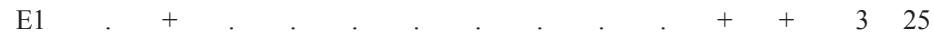

E1 . . . . . . . . . . . . .

E1 . . . . . . . . . . . . .

$\mathrm{E} 1 . .5 .2 . \quad . \quad 1+325$

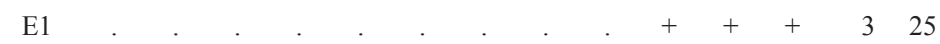

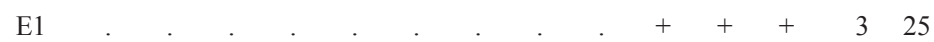

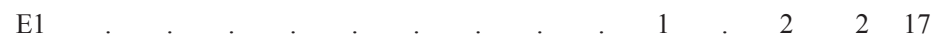

$\mathrm{E} 1$. . . . . . . . . +217

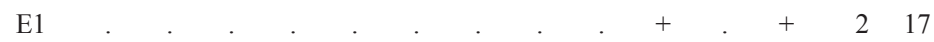

$\mathrm{E} 1$. . . . . . . . . . + . +217

E1

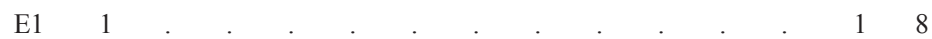


Number of relevé

Allium carinatum subsp. pulchellum

Thymus pulegioides

Hippocrepis comosa

Sanguisorba minor

Hypochoeris maculata

Cirsium x linkianum

Silene vulgaris subsp. vulgaris

Teucrium chamaedrys

Euphorbia cyparissias

Asperula cynanchica

Veronica barrelieri

Stachys recta agg.

TG Trifolio-Geranietea

Arabis pauciflora

Tanacetum corymbosum agg. (T. clusii ?)

Libanotis sibirica subsp. montana

Thalictrum minus

Trifolium rubens

Iris graminea

Polygonatum odoratum

Achillea distans

Vicia sylvatica

Verbascum lanatum

Geranium sanguineum

Thesium bavarum

Origanum vulgare

Digitalis grandiflora

Silene nutans

Verbascum lychnitis

Trifolium medium

Anthericum ramosum

Hypericum perforatum

Valeriana collina

Vincetoxicum hirundinaria

PoT Poo alpinae-Trisetalia

Trollius europaeus

Festuca nigrescens

Anthoxanthum odoratum

Ranunculus nemorosus

Pimpinella major subsp. rubra

Crocus albiflorus

Traunsteinera globosa

Astrantia major

Agrostis capillaris

MA Molinio-Arrhenatheretea

Galium album

Lotus corniculatus s. lat.

Dactylis glomerata s. str.

Lathyrus pratensis

Leontodon hispidus subsp. hispidus

Trifolium pratense

Angelica sylvestris

Vicia sepium

Achillea roseoalba

\begin{tabular}{|c|c|c|c|c|c|c|c|c|c|c|c|c|c|c|}
\hline & 1 & 2 & 3 & 4 & 5 & 6 & 7 & 8 & 9 & 10 & 11 & 12 & Pr. & Fr. \\
\hline E1 & . & . & . & . & . & + & . & . & . & . & . & . & 1 & 8 \\
\hline E1 & . & . & . & . & . & . & . & . & . & + & . & . & 1 & \\
\hline E1 & . & . & . & . & . & . & . & . & . & + & . & . & 1 & \\
\hline E1 & . & . & . & . & . & . & . & . & . & + & . & . & 1 & \\
\hline E1 & . & . & . & . & . & . & . & . & . & . & + & . & 1 & , \\
\hline E1 & . & . & . & . & . & . & . & . & . & . & + & . & 1 & \\
\hline E1 & . & . & . & . & . & . & . & . & . & . & + & . & 1 & $\delta$ \\
\hline E1 & . & . & . & . & . & . & . & . & . & . & . & 2 & 1 & 8 \\
\hline E1 & . & . & . & . & . & . & . & . & . & . & . & + & 1 & \\
\hline E1 & . & . & . & . & . & . & . & . & . & . & . & + & 1 & \\
\hline E1 & . & . & . & . & . & . & . & . & . & . & . & + & 1 & \\
\hline E1 & . & . & . & . & . & . & . & . & . & . & . & + & 1 & \\
\hline
\end{tabular}

$\mathrm{E} 1 .++\quad+\quad 111++1+11192$

$\mathrm{E} 11++1++.+2 .+867$

$\mathrm{E} 1.1+1++.+2 .+467$

E1 111.12 .458

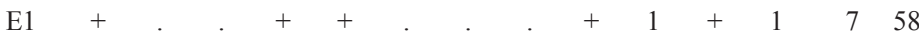

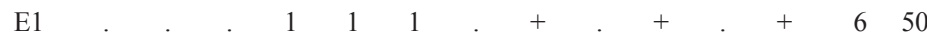

$\mathrm{E} 1$. . . . . . . $+1+433$

$\mathrm{E} 1+\ldots+1 .+1 .+433$

E1 1 . . . . 325

$\mathrm{E} 1+\mathrm{r}+. \quad 325$

$\mathrm{E} 1 \ldots 1+1.25$

$\mathrm{E} 1 .+2.4 .25$

$\mathrm{E} 1+\ldots+\ldots+2 .+217$

E1 . . . . . 2 . 217

E1 . . $+2+217$

E1 . $+2+217$

E1 $+\begin{array}{lll} & 1 & 8 \\ & & \end{array}$

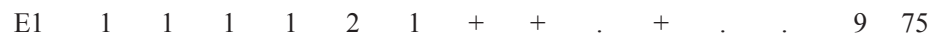

$\begin{array}{lllllllllllllll}\mathrm{E} 1 & 1 & 1 & 1 & 1 & 1 & . & . & . & 1 & 1 & + & 8 & 67\end{array}$

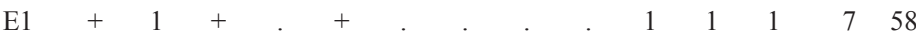

$\mathrm{E} 1+.+1+.+2 .+458$

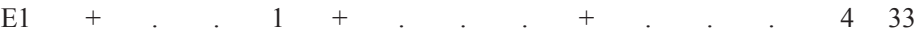

$\mathrm{E} 1 \mathrm{r}++. .2525$

$\mathrm{E} 1++++{ }_{+}+325$

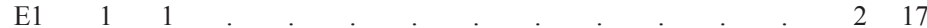

$\mathrm{E} 1++. .5 . \quad . \quad . \quad . \quad 2217$

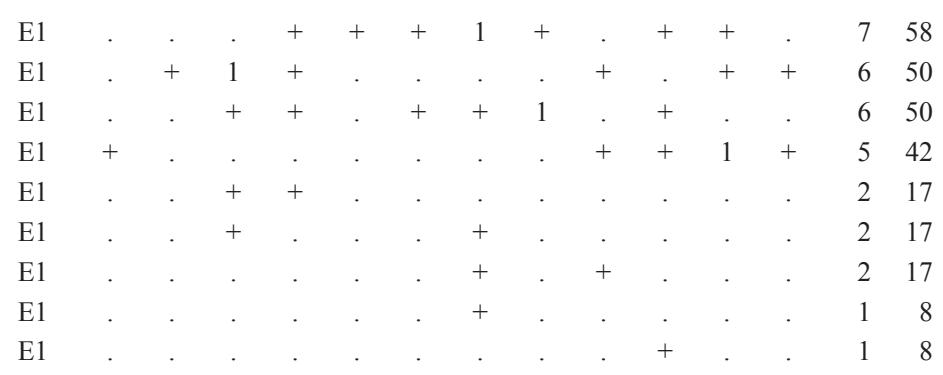




\begin{tabular}{|c|c|c|c|c|c|c|c|c|c|c|c|c|c|c|c|c|}
\hline & Number of relevé & & 1 & 2 & 3 & 4 & 5 & 6 & 7 & 8 & 9 & 10 & 11 & 12 & Pr. & Fr. \\
\hline & Veronica chamaedrys & E1 & . & . & . & . & . & . & . & . & . & . & + & . & 1 & 8 \\
\hline \multirow{5}{*}{ CU } & Calluno-Ulicetea & & & & & & & & & & & & & & & \\
\hline & Potentilla erecta & E1 & + & + & . & . & . & . & . & . & . & 1 & 1 & . & 4 & 33 \\
\hline & Arnica montana & E1 & . & + & . & . & . & . & . & . & . & . & . & . & 1 & \\
\hline & Carex pilulifera & E1 & . & + & . & . & . & . & . & . & . & . & . & . & 1 & \\
\hline & Luzula multiflora & E1 & . & . & + & . & . & . & . & . & . & . & . & . & 1 & 8 \\
\hline \multicolumn{17}{|c|}{ MuAMulgedio-Aconitetea } \\
\hline & Hypericum maculatum & E1 & 1 & + & + & . & . & . & + & . & + & + & + & . & 7 & 58 \\
\hline & Rumex arifolius & E1 & 1 & + & . & . & . & . & + & + & . & + & + & . & 6 & 50 \\
\hline & Lathyrus occidentalis var. montanus & E1 & + & + & + & . & . & . & 2 & + & . & . & . & . & 5 & 42 \\
\hline & Silene vulgaris subsp. antelopum & E1 & . & . & + & . & . & + & 1 & 1 & . & 1 & . & . & 5 & 42 \\
\hline & Pleurospermum austriacum & E1 & . & . & . & + & + & + & + & . & + & . & . & . & 5 & 42 \\
\hline & Veratrum album s. lat. & E1 & 1 & 1 & + & . & + & $\mathrm{r}$ & . & . & . & . & . & . & 5 & 42 \\
\hline & Heracleum sphondylium subsp. montanum & E1 & 2 & . & . & . & . & . & 1 & + & + & . & . & . & 4 & 33 \\
\hline & Polygonatum verticillatum & E1 & . & 1 & . & . & + & 1 & . & 3 & . & . & . & . & 4 & 33 \\
\hline & Thalictrum aquilegiifolium & E1 & $\mathrm{r}$ & . & . & . & . & . & + & + & . & . & . & . & 3 & 25 \\
\hline & Carduus carduelis & E1 & . & . & + & . & . & . & & + & + & . & . & . & 3 & 25 \\
\hline & Senecio ovatus & E1 & + & . & . & . & . & . & . & . & + & . & . & . & 2 & 17 \\
\hline & Chaerophyllum villarsii & E1 & . & + & . & . & . & . & 1 & . & . & . & . & . & 2 & 17 \\
\hline & Silene dioica & E1 & . & . & . & . & + & . & + & . & . & . & . & . & 2 & 17 \\
\hline & Aconitum lycoctonum subsp. ranunculifolium & E1 & . & . & . & . & . & + & 2 & . & . & . & . & . & 2 & 17 \\
\hline & Crepis pyrenaica & E1 & . & . & . & . & . & . & + & + & . & . & . & . & 2 & 17 \\
\hline & Myrrhis odorata & E1 & . & . & . & . & . & . & + & + & . & . & . & . & 2 & 17 \\
\hline & Poa hybrida & E1 & . & . & . & . & . & . & + & 1 & . & . & . & . & 2 & 17 \\
\hline & Allium victorialis & E1 & . & 3 & . & . & . & . & . & . & . & . & . & . & 1 & 8 \\
\hline & Aconitum degenii subsp. paniculatum & E1 & . & . & . & . & . & . & + & . & . & . & . & . & 1 & 8 \\
\hline & Geum rivale & E1 & . & . & . & . & . & . & + & . & . & . & . & . & 1 & 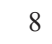 \\
\hline & Pedicularis hacquetii & E1 & . & . & . & . & . & . & + & . & . & . & . & . & 1 & \\
\hline & Ranunculus platanifolius & E1 & . & . & . & . & . & . & + & . & . & . & . & . & 1 & \\
\hline & Eryngium alpinum & E1 & . & . & . & . & . & . & . & 2 & . & . & . & . & 1 & \\
\hline & Sorbus chamaemespilus & E2 & . & . & . & . & . & . & . & 1 & . & . & . & . & 1 & $\gamma$ \\
\hline & Aconitum angustifolium & E1 & . & . & . & . & . & . & . & . & + & . & . & . & 1 & $\gamma$ \\
\hline \multirow[t]{4}{*}{ TR } & Thlaspietea rotundifolii & & & & & & & & & & & & & & & \\
\hline & Ligusticum seguieri & E1 & . & . & . & . & . & . & + & . & + & . & . & 1 & 3 & 25 \\
\hline & Adenostyles glabra & E1 & . & . & . & . & . & . & + & . & . & . & . & . & 1 & 8 \\
\hline & Viola pyrenaica & E1 & . & . & . & . & . & . & . & 1 & . & . & . & . & 1 & 8 \\
\hline \multirow[t]{10}{*}{ AT } & Asplenietea trichomanis & & & & & & & & & & & & & & & \\
\hline & Primula auricula & E1 & . & . & . & . & + & $\mathrm{r}$ & . & . & + & . & . & . & 3 & 25 \\
\hline & Sedum maximum & E1 & . & . & . & . & . & . & . & + & . & . & . & . & 1 & 8 \\
\hline & Dianthus sylvestris & E1 & . & . & . & . & . & . & . & . & + & . & . & . & 1 & 8 \\
\hline & Saxifraga crustata & E1 & . & . & . & . & . & . & . & . & + & . & . & . & 1 & 8 \\
\hline & Saxifraga hostii & E1 & . & . & . & . & . & . & . & . & + & . & . & . & 1 & 8 \\
\hline & Sempervivum tectorum & E1 & . & . & . & . & . & . & . & . & + & . & . & . & 1 & 8 \\
\hline & Silene hayekiana & E1 & . & . & . & . & . & . & . & . & + & . & . & . & 1 & 8 \\
\hline & Asplenium ruta-muraria & E1 & . & . & . & . & . & . & . & . & + & . & . & . & 1 & 8 \\
\hline & Iris pallida subsp. cengialti & E1 & . & . & . & . & . & . & . & . & . & . & . & + & 1 & 8 \\
\hline \multirow[t]{8}{*}{ EP } & Erico-Pinetea & & & & & & & & & & & & & & & \\
\hline & Chamaecytisus hirsutus subsp. ciliatus & E1 & + & + & . & . & . & . & . & . & + & + & + & + & 6 & 50 \\
\hline & Erica carnea & E1 & + & 1 & . & . & . & . & . & . & + & . & . & . & 3 & 25 \\
\hline & Genista radiata & E1 & . & . & . & . & . & 3 & + & 3 & . & . & . & . & 3 & 25 \\
\hline & Calamagrostis varia & E1 & . & . & . & . & 1 & 1 & . & . & . & . & . & . & 2 & 17 \\
\hline & Rubus saxatilis & E1 & . & . & . & . & + & . & + & . & . & . & . & . & 2 & 17 \\
\hline & Polygala chamaebuxus & E1 & . & . & . & . & . & . & . & . & . & + & + & . & 2 & 17 \\
\hline & Carex ornithopoda & E1 & . & + & . & . & . & . & . & . & . & . & . & . & 1 & \\
\hline
\end{tabular}


Number of relevé

Molinia caerulea susbp. arundinacea

E1

1

P Vaccinio-Piceetea

Aposeris foetida

Calamagrostis arundinacea

Luzula luzuloides

Solidago virgaurea

Picea abies

Vaccinium myrtillus

Clematis alpina

Rosa pendulina

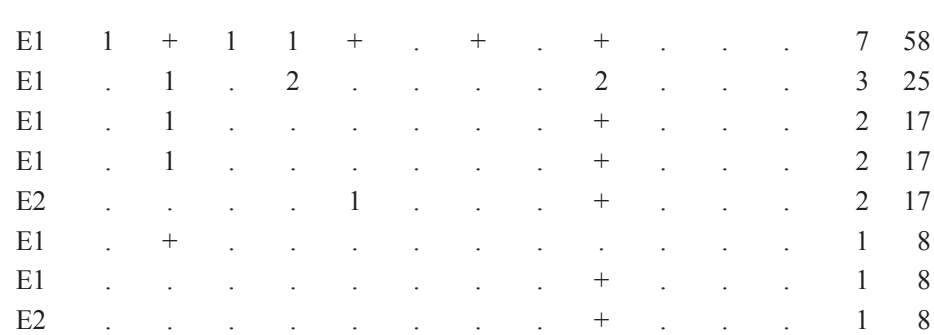

EC Erythronio-Carpinion

Ornithogalum pyrenaicum

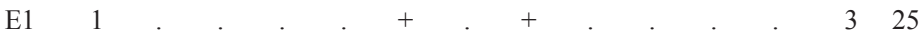

Helleborus odorus

Galanthus nivalis

Primula vulgaris

AF Aremonio-Fagion

Knautia drymeia

FS Fagetalia sylvaticae

Mercurialis perennis

Lilium martagon

Myosotis sylvatica

Campanula trachelium

Galium laevigatum

Acer pseudoplatanus

Lonicera alpigena

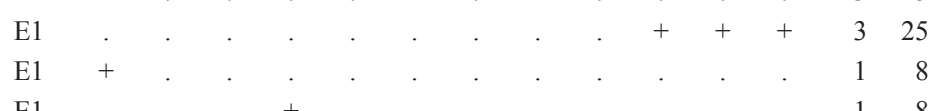

P Quercetalia pubescentis

Melittis melissophyllum

Primula veris subsp. columnae

Peucedanum schottii var. schottii

Convallaria majalis

Orchis mascula subsp. speciosa

Sorbus aria

Peucedanum schottii var. petraeum

Carex flacca

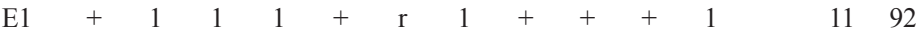

$\mathrm{E} 1+\ldots+1+111+.+975$

$\mathrm{E} 11++.+. .2 . \quad . \quad 433$

$\mathrm{E} 1 .+\ldots+2 .+217$

$\mathrm{E} 1$. . . . . . . . . . . . . 18

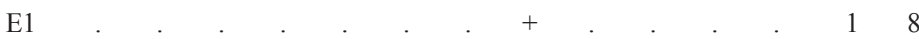

$\mathrm{E} 1$. . . . . . . . . . . . . 18

E2 . . . . . . . . . . . . . 18

QF Querco-Fagetea

Cruciata glabra

Veratrum nigrum

Carex digitata

Corylus avellana

Anemone nemorosa

Listera ovata

Dactylorhiza fuchsii

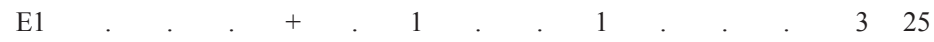

$\mathrm{E} 1$. . . ++ . . . . . . 325

E1 . . . . . . . . . 1 . 1 . 2 . 325

$\mathrm{E} 1$. . . . . . . . . 2 . 217

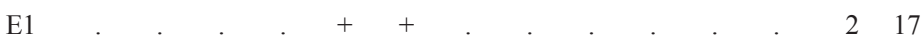

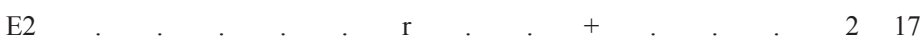

E1

E1

E1 . . . . . . . . $111+325$

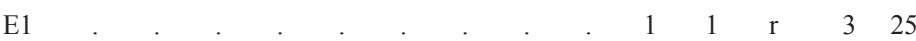

$\mathrm{E} 1 .+.+.+2 .+2.217$

$\mathrm{E} 2$ 2. . . . . . . . . +217

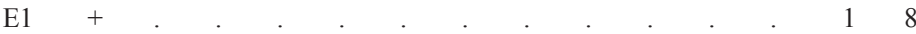

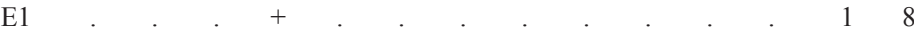

E1 . . . . . . . . . . . . . . 18

RP Rhamno-Prunetea

Rhamnus catharticus

E2a

E2

ML Mosses

Tortella tortuosa

E0

Schistidium apocarpum

E0

Fissidens dubius

E0

A Limestone, D Dolomite, R Chert, L Marl, Gr Gravel, Re Rendzina, Li Lithosol 
Table 3: Centaureo julici-Laserpitietum sileris var. geogr. Pedicularis elongata, the Carnic Alps, W Julian Alps, NE Italy. Tabela 3: Centaureo julici-Laserpitietum sileris var. geogr. Pedicularis elongata, Karnijske in zahodne Julijske Alpe, severovzhodna Italija.

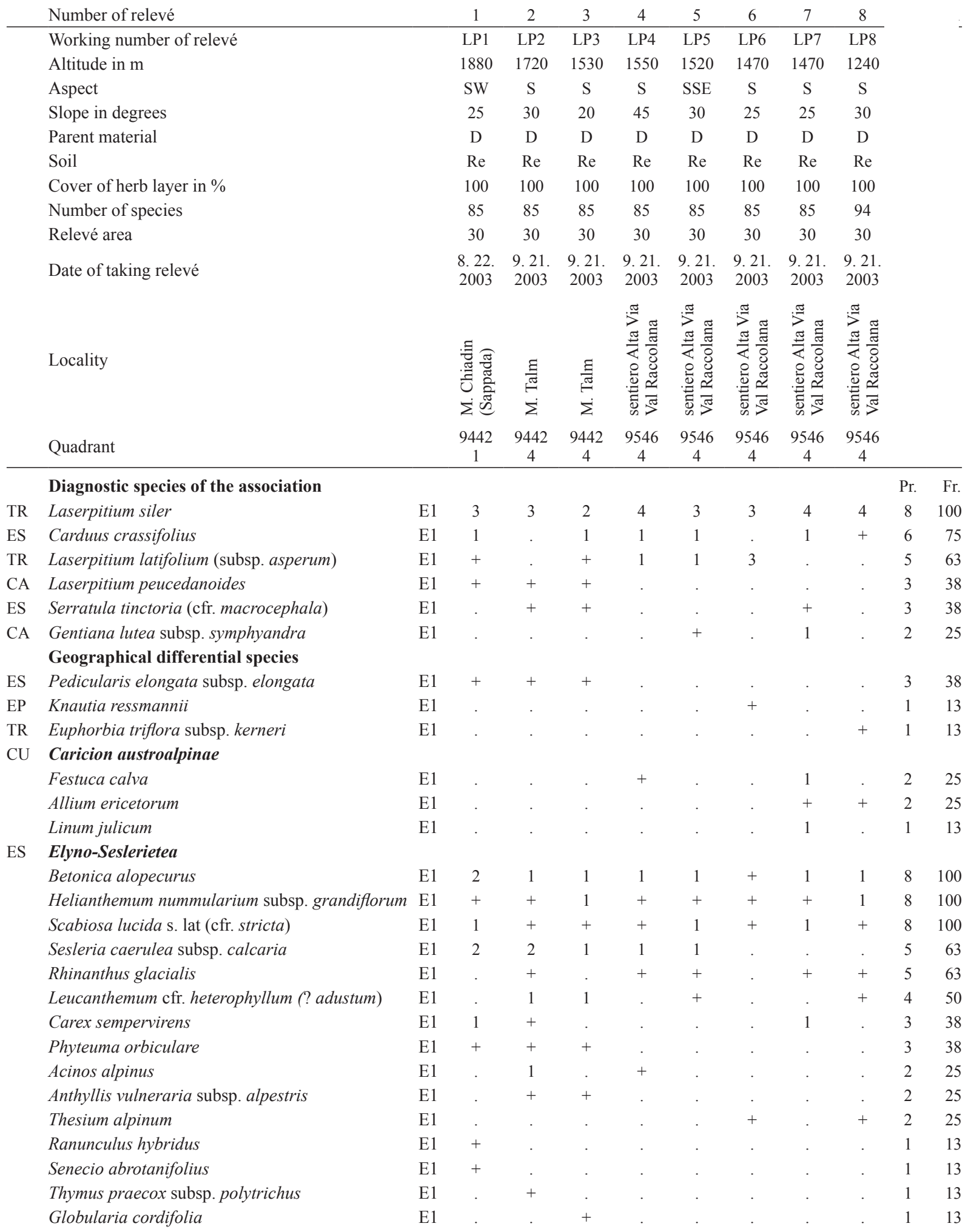


Number of relevé

Helianthemum alpestre

Campanula thyrsoides subsp. carniolica

Galium anisophyllum

Pulsatilla alpina subsp. austroalpina

Senecio doronicum

Polygonum viviparum

FB Festuco-Brometea

Prunella grandiflora

Carex humilis

Bromus transilvanicus

Buphthalmum salicifolium

Koeleria pyramidata s. lat.

Gymnadenia conopsea

Festuca rupicola

Cirsium erisithales

Centaurea bracteata

Phleum phleoides

Asperula cfr. cynanchica

Carex caryophyllea

Brachypodium rupestre

Galium verum

Stachys labiosa

Briza media

Galium lucidum

Bromus erectus s. lat.

Bupleurum ranunculoides subsp. caricinum

Linum viscosum

Peucedanum oreoselinum

Centaurea triumfetti subsp. aligera $(=C$. axilaris $)$

Allium carinatum

Asperula purpurea

Carlina acaulis

Teucrium chamaedrys

TG Trifolio-Geranietea

Valeriana collina

Polygonatum odoratum

Thalictrum minus

Silene nutans s. lat.

Vincetoxicum hirundinaria

Clinopodium vulgare

Thesium bavarum

Viola hirta

Digitalis grandiflora

Origanum vulgare

PaT Poo alpinae-Trisetalia

Ranunculus nemorosus

Festuca nigrescens

MA Molinio-Arrhenatheretea

Lotus corniculatus

Lathyrus pratensis

Trifolium pratense

Vicia cracca

Orobanche minor

Achillea millefolium

Pimpinella major

\begin{tabular}{lll}
1 & 2 & 3 \\
\hline
\end{tabular}

$4 \quad 5$

\section{E1}

E1

E1

E1

E1

$\begin{array}{llllllllll}\text { E1 } & 1 & 1 & 1 & + & + & + & 1 & + & 78\end{array}$

$\begin{array}{lllllllllll}\mathrm{E} 1 & . & 2 & 1 & 1 & 1 & . & 1 & 1 & 6 & 75\end{array}$

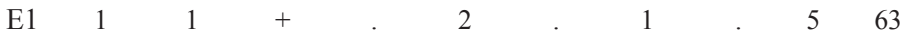

$\mathrm{E} 1++\quad+\quad+\quad . \quad 4 \quad 50$

$\begin{array}{lllllll}\text { E1 } & 1 & 1 & 1 & 1 & 4 & 50\end{array}$

E1 1 . . . . . . . 252

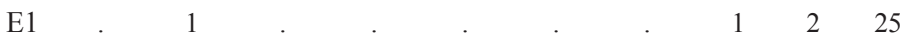

E1 $\quad \begin{array}{lllllllll}1 & 1 & 1 & + & 4 & 50\end{array}$

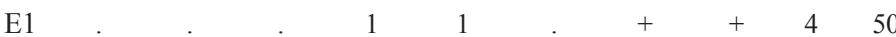

E1 . . . . $\quad \begin{array}{lllllllllll} & 1 & 2 & 1 & . & 3 & 38\end{array}$

E1 . . . . . . . 2 . 25

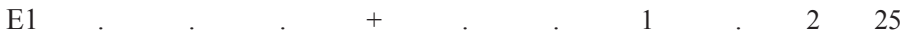

E1 $\ldots+\ldots 25$

E1 $+\quad+\quad 25$

E1 . . . . . . . . . . . . 113

E1

E1 $+t_{13}+13$

$\begin{array}{llll}1 & 1 & 13\end{array}$

E1 . . . . . . . . 113013

E1 . . . . . . . 13013

E1 . . . . . . . 11313

E1

E1

E1

E1

E1

E1

E1

E1

E1

E1

E1

E1

E1

E1

E1

E1

E1

E1

E1

E1

E1

E1

. $.+1+5+53$

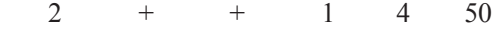

$+\quad+\quad+25$

$+\quad+\quad 13$

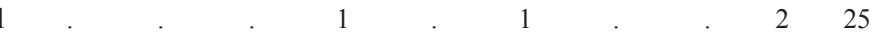

$1 . \quad . \quad . \quad . \quad . \quad . \quad 1313$

. . . . $.4+38$

. . . . . +25

. . . . . . . . . . . . . 113 


\begin{tabular}{|c|c|c|c|c|c|c|c|c|c|c|c|c|}
\hline & Number of relevé & & 1 & 2 & 3 & 4 & 5 & 6 & 7 & 8 & Pr. & Fr. \\
\hline & Veronica chamaedrys & E1 & . & . & . & . & . & + & . & . & 1 & 13 \\
\hline \multirow[t]{3}{*}{$\mathrm{CU}$} & Calluno-Ulicetea & & & & & & & & & & & \\
\hline & Phyteuma zahlbruckneri & E1 & . & . & . & . & . & 1 & + & . & 2 & 25 \\
\hline & Potentilla erecta & E1 & . & . & + & . & . & . & . & . & 1 & 13 \\
\hline \multirow[t]{8}{*}{$\mathrm{MuA}$} & Mulgedio-Aconitetea & & & & & & & & & & & \\
\hline & Silene vulgaris subsp. antelopum & E1 & . & + & + & + & + & + & + & . & 6 & 75 \\
\hline & Heracleum sphondilium subsp. elegans & E1 & . & . & . & 1 & . & 1 & + & . & 3 & 38 \\
\hline & Geranium sylvaticum & E1 & . & . & . & . & 1 & 1 & . & . & 2 & 25 \\
\hline & Hypericum maculatum & E1 & . & . & . & . & . & + & . & . & 1 & 13 \\
\hline & Rumex alpestris & E1 & . & . & . & . & . & + & . & . & 1 & 13 \\
\hline & Thalictrum aquilegifolium & E1 & . & . & . & . & . & + & . & . & 1 & 13 \\
\hline & Lathyrus laevigatus & E1 & . & . & . & . & . & . & 1 & . & 1 & 13 \\
\hline \multirow[t]{2}{*}{ TR } & Thlaspietea rotundifolii & & & & & & & & & & & \\
\hline & Biscutella laevigata & E1 & . & + & . & . & . & . & . & + & 2 & 25 \\
\hline \multirow[t]{3}{*}{ AT } & Asplenietea trichomanis & & & & & & & & & & & \\
\hline & Campanula spicata & E1 & . & + & . & . & . & . & . & . & 1 & 13 \\
\hline & Saxifraga hostii & E1 & . & . & . & + & . & . & . & . & 1 & 13 \\
\hline \multirow[t]{11}{*}{ EP } & Erico-Pinetea & & & & & & & & & & & \\
\hline & Calamagrostis varia & E1 & 2 & 2 & 2 & 1 & 2 & . & 2 & 2 & 7 & 88 \\
\hline & Peucedanum austriacum subsp. rablense & E1 & + & . & . & 1 & 1 & 1 & + & + & 6 & 75 \\
\hline & Erica carnea & E1 & . & + & 1 & + & . & . & 1 & 1 & 5 & 63 \\
\hline & Genista radiata & E1 & . & . & . & 2 & 2 & 1 & 2 & 1 & 5 & 63 \\
\hline & Molinia caerulea subsp. arundinacea & E1 & . & 2 & 2 & . & . & . & 1 & 2 & 4 & 50 \\
\hline & Chamaecytisus purpureus & E1 & . & . & 1 & . & . & . & 1 & 1 & 3 & 38 \\
\hline & Daphne cneorum & E1 & . & 1 & . & . & . & . & + & . & 2 & 25 \\
\hline & Arctostaphylos uva-ursi & E1 & . & . & . & . & + & . & . & . & 1 & 13 \\
\hline & Rubus saxatilis & E1 & . & . & . & . & + & . & . & . & 1 & 13 \\
\hline & Polygala chamaebuxus & E1 & . & . & . & . & . & . & . & 1 & 1 & 13 \\
\hline \multirow[t]{3}{*}{ VP } & Vaccinio-Piceetea & & & & & & & & & & & \\
\hline & Larix decidua & E1 & . & . & . & . & + & . & . & . & 1 & 13 \\
\hline & Rosa pendulina & E1 & . & . & . & . & . & + & . & . & 1 & 13 \\
\hline \multirow[t]{3}{*}{$\mathrm{AF}$} & Aremonio-Fagion & & & & & & & & & & & \\
\hline & Cyclamen purpurascens & E1 & . & . & . & + & . & . & . & . & 1 & 13 \\
\hline & Anemone trifolia & E1 & . & . & . & . & . & . & . & + & 1 & 13 \\
\hline \multirow[t]{5}{*}{ FS } & Fagetalia sylvaticae & & & & & & & & & & & \\
\hline & Mercurialis perennis & E1 & . & . & . & + & + & . & + & + & 4 & 50 \\
\hline & Luzula nivea & E1 & . & . & . & . & + & + & . & . & 2 & 25 \\
\hline & Galium laevigatum & E1 & . & . & . & . & . & + & . & . & 1 & 13 \\
\hline & Poa nemoralis & E1 & . & . & . & . & . & + & . & . & 1 & 13 \\
\hline \multirow[t]{3}{*}{ QP } & Quercetalia pubescentis & & & & & & & & & & & \\
\hline & Carex flacca & E1 & . & 1 & + & . & . & . & . & + & 3 & 38 \\
\hline & Convallaria majalis & E1 & . & . & . & . & . & . & . & 1 & 1 & 13 \\
\hline \multirow[t]{2}{*}{ QF } & Querco-Fagetea & & & & & & & & & & & \\
\hline & Carex digitata & E1 & . & . & . & . & . & . & . & + & 1 & 13 \\
\hline \multirow[t]{2}{*}{$\mathrm{O}$} & Other species & & & & & & & & & & & \\
\hline & Fragaria vesca & E1 & . & . & . & . & . & 1 & . & . & 1 & 13 \\
\hline
\end{tabular}


Table 4: Laserpitio sileri-Grafietum golakae Poldini ass. nov., the Julian Prealps, NE Italy.

Tabela 4: Laserpitio sileri-Grafietum golakae Poldini ass. nov., Julijske Predalpe, severovzhodna Italija.

\begin{tabular}{|c|c|c|c|c|c|c|c|c|c|c|c|c|c|c|c|c|c|}
\hline & Number of relevé & & 1 & 2 & 3 & 4 & 5 & 6 & 7 & 8 & 9 & 10 & 11 & 12 & 13 & & \\
\hline & Working number of relevé & & $\begin{array}{l}\stackrel{2}{2} \\
\stackrel{2}{2} \\
\vec{n}\end{array}$ & $\underset{n}{\mathbb{Z}}$ & 年 & 号 & 恙 & $\stackrel{\infty}{\dot{H}^{\circ}}$ & 守 & in & $\bar{n}$ & oे & in & $\bar{i}$ & $\underset{i}{\mathbb{N}}$ & & \\
\hline & Altitude in $\mathrm{m}$ & & $\begin{array}{l}\stackrel{n}{+} \\
\stackrel{1}{+}\end{array}$ & $\begin{array}{l}\stackrel{\infty}{2} \\
\stackrel{2}{n}\end{array}$ & $\stackrel{?}{\stackrel{9}{O}}$ & 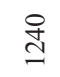 & $\stackrel{\varrho}{\varrho}$ & 움 & $\begin{array}{l}\text { D } \\
-2\end{array}$ & $\underset{\infty}{\infty}$ & $\begin{array}{l}\stackrel{0}{0} \\
\stackrel{0}{2}\end{array}$ & $\stackrel{\circ}{\underset{J}{ \pm}}$ & $\underset{-}{0}$ & 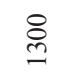 & $\vec{\beth}$ & & \\
\hline & Aspect & & SEE & $\mathrm{S}$ & 0 & 0 & $\mathrm{~S}$ & $\mathrm{~N}$ & $\mathrm{~N}$ & 0 & $\mathrm{SE}$ & $\mathrm{SE}$ & SE & $\mathrm{S}$ & $\mathrm{S}$ & & \\
\hline & Slope in degrees & & 5 & 20 & 0 & 0 & $30-35$ & 15 & 15 & 0 & 10 & 10 & 5 & 30 & 30 & & \\
\hline & Parent material & & $\mathrm{A}$ & A & A & A & A & A & A & A & A & A & A & A & $\mathrm{A}$ & & \\
\hline & Soil & & $\operatorname{Re}$ & $\mathrm{Re}$ & $\mathrm{Re}$ & $\mathrm{Re}$ & $\mathrm{Re}$ & $\mathrm{Re}$ & $\mathrm{Re}$ & $\operatorname{Re}$ & $\operatorname{Re}$ & $\operatorname{Re}$ & $\mathrm{Re}$ & $\mathrm{Re}$ & $\mathrm{Re}$ & & \\
\hline & Cover of herb layer in $\%$ & E1 & 100 & 100 & 100 & 100 & 100 & 100 & 100 & 100 & 100 & 100 & 100 & 100 & 100 & & \\
\hline & Number of species & & 34 & 22 & 15 & 29 & 48 & 39 & 27 & 27 & 27 & 19 & 27 & 14 & 17 & & \\
\hline & Relevé area & $\mathrm{m}^{2}$ & 30 & 30 & 30 & 30 & 30 & 30 & 30 & 30 & 30 & 30 & 30 & 30 & 30 & & \\
\hline & Date of taking relevé & & 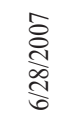 & 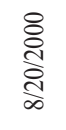 & 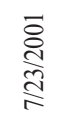 & 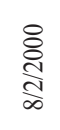 & 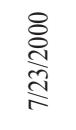 & 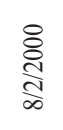 & 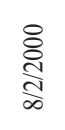 & 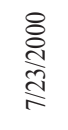 & 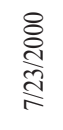 & 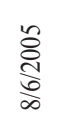 & 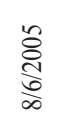 & ఫ્ત & 总 & & \\
\hline & Quadrant & & 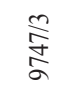 & $\frac{\overline{5}}{5}$ & $\begin{array}{l}\stackrel{N}{\ddagger} \\
\underset{\sigma}{\circ}\end{array}$ & 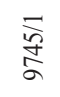 & 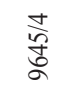 & $\underset{\frac{5}{5}}{\frac{5}{5}}$ & $\frac{\sqrt[s]{5}}{\frac{5}{a}}$ & 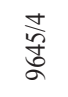 & $\begin{array}{l}\frac{t}{i ⿱ 宀} \\
\text { do }\end{array}$ & $\underset{\frac{m}{\sigma}}{\stackrel{c}{\sigma}}$ & 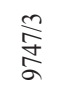 & $\frac{m}{\text { ind }}$ & 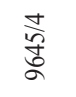 & & \\
\hline & Author of the relevé & & 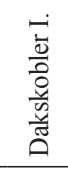 & $\begin{array}{l}\dot{1} \\
\dot{\Xi} \\
\dot{0} \\
0 \\
0\end{array}$ & $\begin{array}{l}\dot{j} \\
\dot{0} \\
\dot{0} \\
\stackrel{0}{0}\end{array}$ & 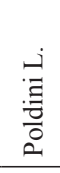 & 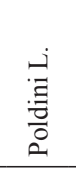 & $\begin{array}{l}\dot{\ddots} \\
\dot{\Xi} \\
\dot{0} \\
0\end{array}$ & $\begin{array}{l}\dot{j} \\
\dot{\Xi} \\
\dot{0} \\
\dot{0}\end{array}$ & $\begin{array}{l}\dot{1} \\
\dot{\Xi} \\
\dot{0} \\
\dot{0} \\
\dot{0}\end{array}$ & 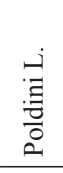 & : & 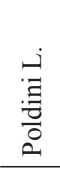 & 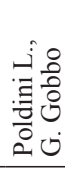 & 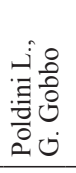 & & \\
\hline & Diagnostic species of the association & & & & & & & & & & & & & & & Pr. & Fr. \\
\hline MuA & Grafia golaka & E1 & 4 & 3 & 4 & 4 & 1 & 4 & 3 & 2 & 1 & 4 & 4 & 2 & 3 & 13 & 100 \\
\hline TG & Laserpitium siler & E1 & . & 3 & . & . & 4 & . & . & 4 & 3 & . & . & 4 & 4 & 6 & 46 \\
\hline MuA & Molopospermum peloponnesiacum subsp. bauhinii & E1 & $\mathrm{r}$ & . & . & 1 & . & . & . & . & 1 & . & + & . & . & 4 & 31 \\
\hline $\mathrm{CA}$ & Laserpitium peucedanoides & E1 & . & . & . & . & + & + & 1 & . & . & . & . & . & . & 3 & 23 \\
\hline \multirow{18}{*}{ MuA } & Mulgedio-Aconitetea & & & & & & & & & & & & & & & & \\
\hline & Veratrum album subsp. lobelianum & E1 & 1 & . & . & + & + & + & 1 & . & . & 1 & 1 & . & + & 8 & 62 \\
\hline & Aconitum lycoctonum subsp. ranunculifolium & E1 & + & . & . & 1 & 1 & + & . & . & 1 & . & + & . & 2 & 7 & 54 \\
\hline & Hypericum maculatum & E1 & 1 & . & . & . & . & . & . & . & . & + & . & + & . & 3 & 23 \\
\hline & Heracleum sphondylium subsp. montanum & E1 & $\mathrm{r}$ & . & . & . & + & . & . & . & . & . & . & . & + & 3 & 23 \\
\hline & Polygonatum verticillatum & E1 & . & . & . & 1 & . & 1 & 1 & . & . & . & . & . & . & 3 & 23 \\
\hline & Aconitum tauricum (inc. A. angustifolium) & E1 & $\mathrm{r}$ & . & . & . & . & . & . & . & . & . & + & . & . & 2 & 15 \\
\hline & Senecio ovatus & E1 & . & . & . & . & . & . & . & . & . & . & + & 1 & . & 2 & 15 \\
\hline & Alchemilla xanthochlora & E1 & 1 & . & . & . & . & . & . & . & . & . & . & . & . & 1 & 8 \\
\hline & Geum rivale & E1 & 1 & . & . & . & . & . & . & . & . & . & . & . & . & 1 & 8 \\
\hline & Rumex arifolius & E1 & + & . & . & . & . & . & . & . & . & . & . & . & . & 1 & 8 \\
\hline & Rumex alpinus & E1 & . & + & . & . & . & . & . & . & . & . & . & . & . & 1 & 8 \\
\hline & Pleurospermum austriacum & E1 & . & . & . & 1 & . & . & . & . & . & . & . & . & . & 1 & 8 \\
\hline & Ranunculus platanifolius & E1 & . & . & . & + & . & . & . & . & . & . & . & . & . & 1 & 8 \\
\hline & Salix glabra & E1 & . & . & . & + & . & . & . & . & . & . & . & . & . & 1 & 8 \\
\hline & Stemmacantha rhapontica subsp. rhapontica & E1 & . & . & . & . & . & + & . & . & . & . & . & . & . & 1 & 8 \\
\hline & Salix appendiculata & E2 & . & . & . & . & . & . & . & + & . & . & . & . & . & 1 & 8 \\
\hline & Dactylis glomerata subsp. slovenica & E1 & . & . & . & . & . & . & . & . & . & 1 & . & . & . & 1 & 8 \\
\hline \multirow[t]{6}{*}{ TG } & Trifolio-Geranietea & & & & & & & & & & & & & & & & \\
\hline & Vincetoxicum hirundinaria & E1 & . & . & + & + & 1 & . & . & 1 & 1 & . & . & + & 1 & 7 & 54 \\
\hline & Thalictrum minus & E1 & . & . & . & + & . & + & . & + & 1 & . & + & . & . & 5 & 38 \\
\hline & Anthericum ramosum & E1 & . & . & . & . & 1 & . & . & + & 1 & . & . & . & 1 & 4 & 31 \\
\hline & Libanotis sibirica subsp. montana & E1 & . & . & . & . & + & 1 & 1 & . & + & . & . & . & . & 4 & 31 \\
\hline & Polygonatum odoratum & E1 & . & . & . & . & 1 & . & . & + & 1 & . & . & . & . & 3 & 23 \\
\hline
\end{tabular}


Number of relevé

Achillea distans

Iris graminea

Trifolium rubens

Verbascum lanatum

Thesium bavarum

Trifolium medium

Clinopodium vulgare

Inula spiraeifolia

Silene nutans

Hypericum perforatum

Petasites paradoxus

Laserpitium latifolium

Valeriana collina ( $V$. wallrothii)

Digitalis grandiflora

Vicia sylvatica

Libanotis daucifolia

FB Festuco-Brometea

Buphthalmum salicifolium

Brachypodium rupestre s. lat.

Cirsium erisithales

Scabiosa columbaria

Dianthus monspessulanus

Briza media

Carlina acaulis subsp. simplex

Centaurea triumfettii

Galium verum

Peucedanum oreoselinum

Bromopsis transsilvanica

Trifolium montanum

Campanula glomerata

Allium carinatum subsp. carinatum

Asphodelus albus

Gymnadenia conopsea

Ranunculus polyanthemophyllus

Bromopsis erecta

Helictotrichon praeustum

Stachys labiosa

Thymus pulegioides

Veronica teucrium

Euphorbia cyparissias

Centaurea scabiosa subsp. fritschii

Cirsium pannonicum

Cirsium x linkianum

Campanula rotundifolia

Centaurea bracteata

CA Caricion austroalpinae

Scorzonera rosea

Koeleria eriostachya

Centaurea haynaldii subsp. julica

Festuca calva

ES Elyno-Seslerietea

Rhinanthus aristatus

Betonica alopecuros

Leucanthemum heterophyllum

Sesleria caerulea subsp. calcaria

\begin{tabular}{|c|c|c|c|c|c|c|c|c|c|c|c|c|c|c|c|}
\hline & 1 & 2 & 3 & 4 & 5 & 6 & 7 & 8 & 9 & 10 & 11 & 12 & 13 & Pr. & Fr. \\
\hline E1 & 2 & . & . & . & . & . & & & . & . & + & . & . & 2 & 15 \\
\hline E1 & . & 1 & & . & + & . & & & . & . & . & . & . & 2 & 15 \\
\hline E1 & . & . & . & . & 1 & . & & - & + & . & . & . & . & 2 & 15 \\
\hline E1 & $\mathrm{r}$ & . & . & . & . & . & . & $\cdot$ & . & . & . & . & . & 1 & 8 \\
\hline E1 & . & . & + & . & . & . & $\cdot$ & . & . & . & . & . & . & 1 & 8 \\
\hline E1 & . & . & + & . & . & . & . & . & . & . & . & . & . & 1 & 8 \\
\hline E1 & . & . & . & . & + & . & . & . & . & . & . & . & . & 1 & 8 \\
\hline E1 & . & . & . & . & + & . & & . & . & . & . & . & . & 1 & 8 \\
\hline E1 & . & . & . & . & + & . & 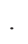 & . & . & . & . & . & . & 1 & 8 \\
\hline E1 & . & . & . & . & . & + & . & . & . & . & . & . & . & 1 & 8 \\
\hline E1 & . & . & . & . & . & . & . & 1 & . & . & . & . & . & 1 & 8 \\
\hline E1 & . & . & . & . & . & . & . & 2 & . & . & . & . & . & 1 & 8 \\
\hline E1 & . & . & . & . & . & . & . & . & + & . & . & . & . & 1 & 8 \\
\hline E1 & . & . & . & . & . & . & . & . & + & . & . & . & . & 1 & 8 \\
\hline E1 & . & . & . & . & . & . & . & . & . & . & + & . & . & 1 & 8 \\
\hline E1 & . & . & . & . & . & . & & . & . & . & . & . & + & 1 & \\
\hline
\end{tabular}

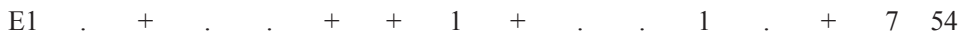

$\mathrm{E} 1$. $\quad . \quad \begin{array}{ccccccccccccc} & 1 & 2 & 1 & 1 & . & 1 & 2 & . & . & . & 6 & 46\end{array}$

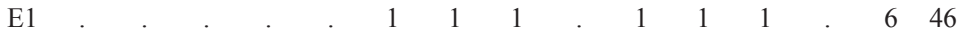

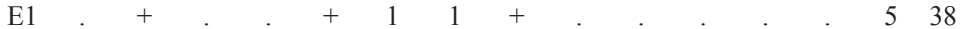

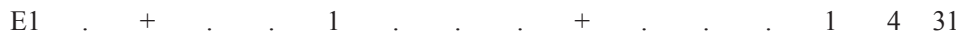

$\mathrm{E} 11 .+. .+. .6323$

$\mathrm{E} 1+\ldots+1.523$

$\mathrm{E} 1 .+\ldots+.+2 .+23$

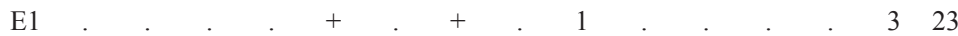

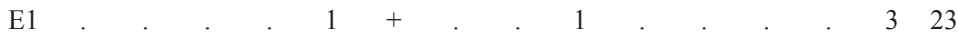

E1 . 2 . . . 1 . . . . . . . 215

$\mathrm{E} 1 .+2 .+.+2.215$

$\mathrm{E} 1 .+\ldots+215$

$\mathrm{E} 1 .+.+2 .+2.215$

E1 1 . . . . . . . . . . . . . 18

$\mathrm{E} 1 .+\ldots .+. . .618$

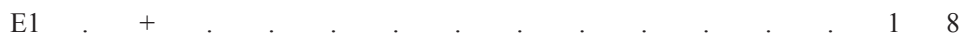

$\mathrm{E} 1$. . . 1 . . . . . . . . . 18

$\mathrm{E} 1$. . . . + . . . . . . . . 18

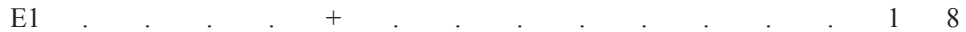

$\mathrm{E} 1$. . . . + . . . . . . . 18

E1

E1

E1

E1

E1

E1

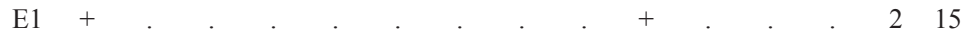

$\mathrm{E} 1.1 .5 .2 .2 .215$

$\mathrm{E} 1 .+1.2 .2 .215$

E1 . . . . . . $1+215$

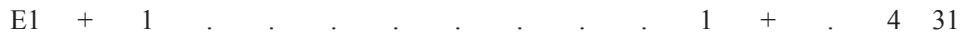

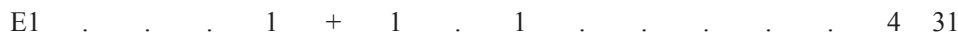

$\mathrm{E} 1 .+.+2 .+2.2215$

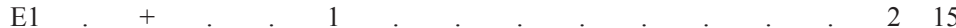


Number of relevé

Galium anisophyllum

Carduus crassifolius s. lat.

Phleum hirsutum

Helianthemum nummularium subsp. grandiflorum

Pimpinella alpina

Carduus defloratus

Leucanthemum adustum

Carex sempervirens

Scabiosa lucida subsp. lucida

PaT Poo alpinae-Trisetalia

Agrostis capillaris

Trollius europaeus

Campanula scheuchzeri

Festuca nigrescens

Ranunculus nemorosus

Traunsteinera globosa

Astrantia major subsp. carinthiaca

Cerastium fontanum

MA Molinio-Arrhenetheretea

Angelica sylvestris

Dactylis glomerata s.str.

Lathyrus pratensis

Galium album

Trifolium pratense

Deschampsia cespitosa

Rumex acetosa

Stellaria graminea

Vicia cracca

Leontodon hispidus

Tragopogon orientalis

Festuca rubra

CU Calluno-Ulicetea

Potentilla erecta

Phyteuma zahlbruckneri

Danthonia decumbens

TR Thlaspietea rotundifolii

Adenostyles glabra

Astrantia carniolica

EP

Erico-Pinetea

Rubus saxatilis

Calamagrostis varia

Chamaecytisus purpureus

Molinia caerulea subsp. arundinacea

Erica carnea

Peucedanum austriacum subsp. rablense

Genista radiata

Carex alba

VP Vaccinio-Piceetea

Calamagrostis arundinacea

Gentiana asclepiadea

Luzula luzuloides

Rosa pendulina

AF Aremonio-Fagion

Knautia drymeia

$\begin{array}{lllllllllllllll}1 & 2 & 3 & 4 & 5 & 6 & 7 & 8 & 9 & 10 & 11 & 12 & 13 & \text { Pr. } & \text { Fr. }\end{array}$

E1

E1

E1

E1

E1

E1

E1

E1

E1

$\mathrm{E} 1+$. . . . . . $+.+1+538$

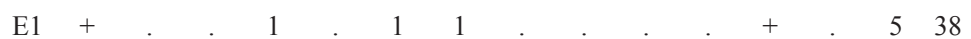

$\mathrm{E} 1 .++.+2 .+2 .+538$

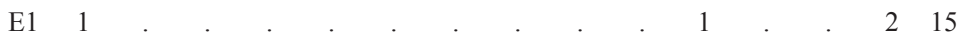

$\mathrm{E} 1+$. . . . . . . . . . . 2 . 215

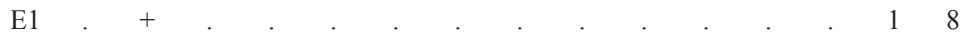

$\mathrm{E} 1$. . . + . . . . . . . . . . . 18

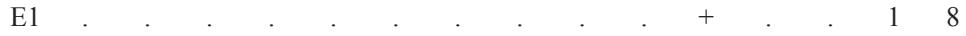

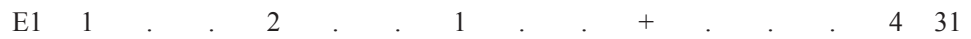

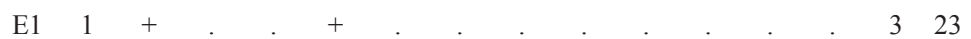

$\mathrm{E} 1$. . . . . . . . . 323

$\mathrm{E} 1$. . . . . . . . . . . 2 . 215

$\mathrm{E} 1$. . . . . + . . . . + . . 215

$\mathrm{E} 1+$. . . . . . . . . . . . . 118

$\mathrm{E} 1+$. . . . . . . . . . . . 118

$\mathrm{E} 1+$. . . . . . . . . . . . 18

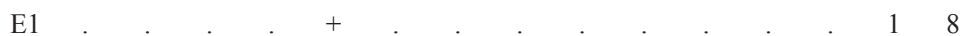

$\mathrm{E} 1$. . . . . . . . . . . . 18

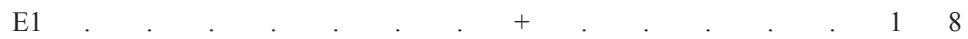

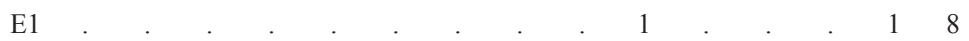

$\mathrm{E} 1++$. . . . . . . . . . . 2215

$\mathrm{E} 1+$. . . . . . . . . . . . . 118

E1 . . . . . . . . . . . . . . . . .

$\mathrm{E} 1$. . . . . . . . . . . . . . . . 18

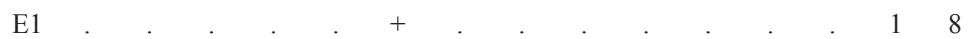

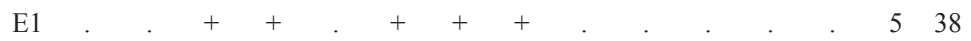

$\mathrm{E} 1$. . $1.1+1.5 .538$

$\mathrm{E} 1$. . ..++2.2 .215$

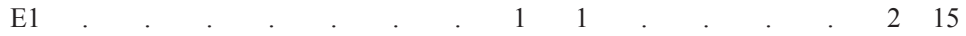

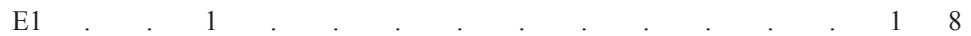

$\mathrm{E} 1$. . . . . . . . . . . . . . . 18

$\mathrm{E} 1$. . . . . . . . . . . 1 . 18

E1 . . . . . . . . . . . 118

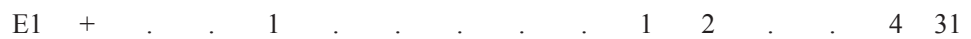

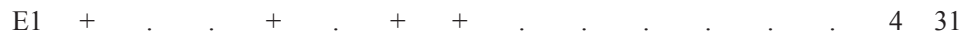

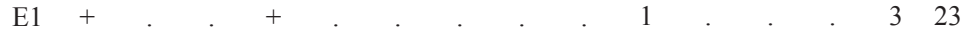

$\mathrm{E} 1 .+.+. .2 .2 .215$

E1 $1.11+1 . \quad 1 .+1.862$ 
Number of relevé

\section{FS Fagetalia sylvaticae}

Lilium martagon

Mercurialis perennis

Heracleum sphondylium subsp. sphondylium

Luzula nivea

Lathyrus vernus

Laburnum alpinum

Acer pseudoplatanus

Melica nutans

Symphytum tuberosum subsp. angustifolium

Galeobdolon flavidum

Fraxinus excelsior

Daphne mezereum

QP Quercetalia pubescentis

Convallaria majalis

Carex flacca

Peucedanum schottii var. schottii

QF Querco-Fagetea

Serratula tinctoria subsp. tinctoria

Dactylorhiza fuchsii

Helleborus odorus

Cruciata glabra

Festuca gigantea

O Other species

Rubus idaeus

Potentilla recta

Salix eleagnos
A Limestone
Re Rendzina

\section{Locality:}

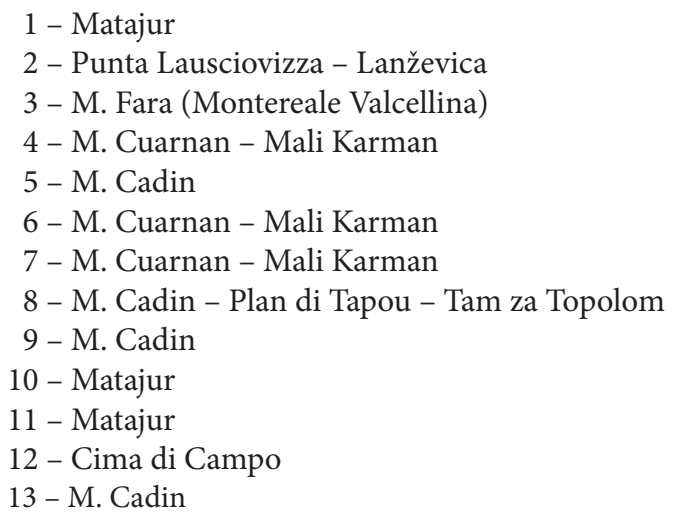

\begin{tabular}{|c|c|c|c|c|c|c|c|c|c|c|c|c|c|c|}
\hline E1 & + & . & . & . & + & + & + & . & . & . & & . & + & 5 \\
\hline E1 & . & . & + & . & + & 1 & + & & . & . & . & . & . & 4 \\
\hline E1 & . & . & . & . & . & . & . & e & 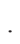 & 1 & 1 & + & & 3 \\
\hline E1 & . & + & . & + & . & . & . & . & . & e & & . & & 2 \\
\hline E1 & . & . & + & . & . & . & . & + & . & . & . & $\cdot$ & & 2 \\
\hline E1 & . & . & + & . & . & . & . & . & . & . & . & . & & 1 \\
\hline E1 & . & . & + & . & . & . & . & . & 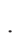 & . & . & . & & 1 \\
\hline E1 & . & . & . & + & . & . & . & . & . & . & . & . & & 1 \\
\hline E1 & . & . & . & + & . & . & . & . & . & . & . & . & & 1 \\
\hline E1 & . & . & . & . & . & + & . & . & $\cdot$ & . & . & . & & 1 \\
\hline E1 & . & . & . & . & . & . & . & + & . & . & . & . & & 1 \\
\hline E1 & . & . & . & . & . & . & . & . & . & . & + & . & & 1 \\
\hline E1 & . & . & + & . & + & . & 1 & . & & . & & . & & 4 \\
\hline E1 & . & . & . & + & . & + & . & . & . & . & . & . & . & 2 \\
\hline E1 & . & . & . & . & + & + & . & . & . & . & . & . & & 2 \\
\hline E1 & . & . & + & . & + & . & + & + & . & . & . & . & 1 & 5 \\
\hline E1 & . & . & . & + & . & . & . & + & . & . & . & . & . & 2 \\
\hline E1 & . & . & . & . & + & + & . & . & . & . & . & . & . & 2 \\
\hline E1 & . & . & . & . & . & + & + & . & . & . & . & . & . & 2 \\
\hline E1 & . & . & . & + & . & . & . & . & . & . & . & . & . & 1 \\
\hline E2 & 2 & 1 & + & . & + & . & . & & . & 2 & + & 1 & & 7 \\
\hline E1 & . & . & . & . & . & . & 1 & . & . & 1 & . & . & . & 2 \\
\hline E1 & . & . & . & . & . & . & . & + & . & . & . & . & . & 1 \\
\hline
\end{tabular}


Table 5: Synoptyc table of the tall herb and fringe communities with the dominant Laserpitium siler or Grafia golaka in NE Italy and Slovenia.

Tabela 5: Sintezna tabela združb visokih steblik in gozdnih robov z dominantnima vrstama Laserpitium siler ali Grafia golaka v severovzhodni Italiji in Sloveniji.

Successive number (Zaporedna številka)

Number of relevés (Število popisov)

\begin{tabular}{|c|c|c|c|c|c|c|}
\hline 1 & 2 & 3 & 4 & 5 & 6 & 7 \\
\hline 31 & 12 & 26 & 8 & 13 & 10 & 10 \\
\hline $\begin{array}{l}\overrightarrow{\widetilde{n}} \\
\overrightarrow{0}\end{array}$ & 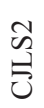 & 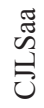 & $\begin{array}{l}\overrightarrow{3} \\
\overrightarrow{3} \\
\overrightarrow{3}\end{array}$ & $\overbrace{0.0}^{n}$ & D্ & 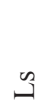 \\
\hline
\end{tabular}

CA Caricion austroalpinae

Centaurea haynaldii subsp. julica

Gentiana lutea subsp. symphyandra

Festuca calva

Laserpitium peucedanoides

Scorzonera rosea

Allium ericetorum

Asperula aristata

Linum julicum

Pedicularis elongata subsp. julica

Heracleum austriacum subsp. siifolium

Gentiana lutea subsp. vardjanii

ES Elyno-Seslerietea

Betonica alopecuros

Sesleria caerulea subsp. calcaria

Carduus crassifolius

Helianthemum nummularium subsp. grandiflorum

Scabiosa lucida subsp. stricta (inc. subsp. lucida)

Serratula tinctoria subsp. macrocephala

Carex sempervirens

Phyteuma orbiculare

Campanula witasekiana

Phleum hirsutum

Pulsatilla alpina subsp. austroalpina

Leucanthemum adustum (inc. L. heterophyllum)

Thymus praecox subsp. polytrichus

Cerastium strictum

Acinos alpinus

Anthyllis vulneraria subsp. alpestris

Galium anisophyllum

Ranunculus montanus

Rhinanthus aristatus

Globularia cordifolia

Astrantia bavarica

Hieracium villosum

Polygonum viviparum

Thesium alpinum

Pimpinella alpina

Potentilla crantzii
E1

E1

E1

E1

E1

E1

E1

E1

E1

E1

E1

E1

E

E1

E1

E1

E1

E1

E1

E1

E1

E1

E1

E1

E

E

E

E

E

E

E

E1

E1

E1

E1

E1

E1

E1

E1

E1

E1

E1

E1

$\begin{array}{rr}85 & \\ 88 & 25 \\ 15 & 25 \\ 38 & 38 \\ 23 & \\ 62 & 25 \\ . & \\ 4 & 13 \\ . & \\ . & \\ . & \end{array}$

$96-100$

$81 \quad 63$

$42 \quad 75$

$81 \quad 100$

$27 \quad 100$

838

. 38

$62 \quad 38$

46

19

.

13

50

13

77

.

25

25

13

15

63

13

31

$31 \quad 100$

$15 \quad 80$

$15 \quad 70$

8

8

8

. 90

15

23

Campanula thyrsoides (inc. subsp. carniolica)

Bartsia alpina

Polygala alpestris

Achillea clavenae 


\begin{tabular}{|c|c|c|c|c|c|c|c|c|c|}
\hline & Successive number & & 1 & 2 & 3 & 4 & 5 & 6 & 7 \\
\hline & Crepis alpestris & E1 & 6 & . & . & . & . & . & - \\
\hline & Gentianella anisodonta & E1 & 6 & . & . & . & . & . & . \\
\hline & Orobanche reticulata & E1 & 6 & . & . & . & . & & . \\
\hline & Astragalus penduliflorus & E1 & 3 & 33 & 23 & . & . & . & . \\
\hline & Carex ferruginea & E1 & 3 & 33 & . & . & . & . & \\
\hline & Anemone narcissiflora & E1 & 3 & 25 & . & . & . & . & . \\
\hline & Senecio abrotanifolius & E1 & 3 & . & . & 13 & . & . & . \\
\hline & Alchemilla fissa & E1 & 3 & & & & . & & . \\
\hline & Androsace villosa & E1 & 3 & . & . & . & . & . & . \\
\hline & Aster alpinus & E1 & 3 & . & . & . & . & . & . \\
\hline & Carex mucronata & E1 & 3 & . & . & . & . & . & . \\
\hline & Gentiana clusii & E1 & 3 & . & . & . & . & . & . \\
\hline & Globularia nudicaulis & E1 & 3 & . & . & . & . & . & . \\
\hline & Hieracium pilosum & E1 & 3 & . & . & . & . & . & . \\
\hline & Myosotis alpestris & E1 & 3 & . & . & . & . & . & . \\
\hline & Oxytropis neglecta & E1 & 3 & . & . & . & . & . & . \\
\hline & Thesium pyrenaicum & E1 & 3 & . & . & . & . & . & . \\
\hline & Carduus defloratus & E1 & . & 8 & . & . & 8 & . & . \\
\hline & Crepis bocconi & E1 & . & 8 & . & . & . & . & . \\
\hline & Pedicularis elongata & E1 & . & . & . & 38 & . & . & . \\
\hline & Helianthemum alpestre & E1 & . & . & . & 13 & . & . & . \\
\hline & Ranunculus hybridus & E1 & . & . & . & 13 & . & . & . \\
\hline & Senecio doronicum & E1 & . & . & . & 13 & 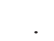 & & . \\
\hline & Sesleria juncifolia subsp. kalnikensis & E1 & . & . & . & . & . & . & 10 \\
\hline \multirow[t]{27}{*}{ FB } & Festuco-Brometea & & & & & & & & \\
\hline & Buphthalmum salicifolium & E1 & 94 & 83 & 62 & 50 & 54 & 50 & 90 \\
\hline & Bromopsis transsilvanica & E1 & 90 & 83 & 96 & 63 & 15 & & \\
\hline & Carex humilis & E1 & 90 & 42 & 96 & 75 & . & 10 & 10 \\
\hline & Koeleria pyramidata s. lat. (inc. K. eryostachia) & E1 & 77 & 58 & 42 & 50 & 15 & 50 & 60 \\
\hline & Gymnadenia conopsea & E1 & 74 & 50 & 19 & 25 & 8 & 10 & 60 \\
\hline & Prunella grandiflora & E1 & 74 & 50 & 50 & 88 & . & . & \\
\hline & Cirsium erisithales & E1 & 71 & 75 & 38 & 50 & 46 & 50 & 30 \\
\hline & Carlina acaulis s. lat. & E1 & 55 & 83 & 77 & 13 & 23 & 10 & 60 \\
\hline & Linum viscosum & E1 & 55 & 33 & 96 & 13 & . & . & \\
\hline & Dianthus monspessulanus & E1 & 52 & 50 & 38 & . & 31 & 10 & \\
\hline & Centaurea triumfettii & E1 & 48 & 50 & 19 & 13 & 23 & 90 & 20 \\
\hline & Campanula glomerata & E1 & 35 & 50 & 69 & . & 15 & 50 & \\
\hline & Thlaspi praecox & E1 & 32 & 8 & . & . & . & . & . \\
\hline & Stachys recta agg. (inc. S. labiosa) & E1 & 29 & 8 & 19 & 13 & 8 & 50 & 30 \\
\hline & Hippocrepis comosa & E1 & 23 & 8 & 4 & . & . & 10 & 30 \\
\hline & Allium senescens & E1 & 19 & . & . & . & . & . & . \\
\hline & Briza media & E1 & 19 & 42 & 27 & 13 & 23 & 40 & 40 \\
\hline & Galium lucidum & E1 & 19 & 17 & 4 & 13 & . & 100 & 50 \\
\hline & Arabis hirsuta & E1 & 13 & . & . & . & . & 10 & . \\
\hline & Allium carinatum subsp. pulchellum & E1 & 10 & 8 & 62 & . & . & . & . \\
\hline & Teucrium chamaedrys & E1 & 10 & 8 & 31 & 13 & . & 30 & 20 \\
\hline & Cuscuta epithymum & E1 & 6 & . & . & . & . & . & . \\
\hline & Euphorbia cyparissias & E1 & 6 & 8 & 58 & . & 8 & 40 & 10 \\
\hline & Helictotrichon praeustum & E1 & 6 & 25 & . & . & 8 & . & \\
\hline & Orobanche gracilis & E1 & 6 & . & . & . & . & . & . \\
\hline & Brachypodium rupestre & E1 & 3 & 50 & 96 & 25 & 46 & 90 & 10 \\
\hline
\end{tabular}




\begin{tabular}{|c|c|c|c|c|c|c|c|c|}
\hline Successive number & & 1 & 2 & 3 & 4 & 5 & 6 & 7 \\
\hline Trifolium montanum & E1 & 3 & 25 & 42 & . & 15 & . & 60 \\
\hline Plantago media & E1 & 3 & 17 & 12 & . & . & . & 50 \\
\hline Peucedanum oreoselinum & E1 & 3 & 17 & . & 13 & 23 & 20 & 50 \\
\hline Thymus pulegioides & E1 & 3 & 8 & . & . & 8 & . & . \\
\hline Sanguisorba minor & E1 & 3 & 8 & . & . & . & . & 10 \\
\hline Dactylorhiza sambucina & E1 & 3 & . & 4 & . & . & . & . \\
\hline Salvia pratensis & E1 & 3 & . & . & . & . & 30 & 50 \\
\hline Pimpinella saxifraga & E1 & 3 & . & . & . & . & 30 & 20 \\
\hline Polygala comosa & E1 & 3 & . & . & . & . & . & 80 \\
\hline Teucrium montanum & E1 & 3 & . & . & . & . & . & . \\
\hline Carlina vulgaris & E1 & 3 & . & . & . & . & . & . \\
\hline Cirsium pannonicum & E1 & . & 25 & 27 & . & 8 & . & 80 \\
\hline Inula hirta & E1 & . & 25 & 58 & . & . & . & . \\
\hline Galium verum & E1 & . & 25 & . & 25 & 23 & 20 & 50 \\
\hline Allium carinatum subsp. carinatum & E1 & . & 25 & . & 13 & 15 & 10 & . \\
\hline Iris sibirica subsp. erirrhiza & E1 & . & 25 & . & . & . & . & . \\
\hline Helianthemum nummularium subsp. obscurum & E1 & . & 17 & . & . & . & 10 & 90 \\
\hline Veronica teucrium & E1 & . & 17 & . & . & 8 & . & . \\
\hline Hypochoeris maculata & E1 & . & 8 & 35 & . & . & . & 10 \\
\hline Cirsium x linkianum & E1 & . & 8 & 23 & . & 8 & . & . \\
\hline Silene vulgaris subsp. vulgaris & E1 & . & 8 & 4 & . & . & 60 & 10 \\
\hline Asperula cynanchica & E1 & . & 8 & . & 25 & . & 60 & 70 \\
\hline Veronica barrelieri (Pseudolysimachion barrelieri) & E1 & . & 8 & . & . & . & . & . \\
\hline Asphodelus albus & E1 & . & . & 100 & . & 8 & . & . \\
\hline Veronica jacquinii & E1 & . & . & 27 & . & . & 40 & 20 \\
\hline Galium purpureum & E1 & . & . & 12 & 13 & . & . & . \\
\hline Euphorbia verrucosa & E1 & . & . & 4 & . & . & 90 & 40 \\
\hline Centaurea bracteata & E1 & . & . & . & 50 & 8 & . & . \\
\hline Phleum phleoides & E1 & . & . & . & 38 & . & . & . \\
\hline Festuca rupicola & E1 & . & . & . & 25 & . & 20 & 80 \\
\hline Carex caryophillea & E1 & . & . & . & 25 & . & . & . \\
\hline Bromopsis erecta & E1 & . & . & . & 13 & 8 & 50 & 90 \\
\hline Bupleurum ranunculoides subsp. caricinum & E1 & . & . & . & 13 & . & . & . \\
\hline Scabiosa columbaria & E1 & . & . & . & . & 38 & . & · \\
\hline Centaurea scabiosa (inc. subsp. fritschii) & E1 & . & . & . & . & 8 & 10 & 40 \\
\hline Campanula rotundifolia & E1 & . & . & . & . & 8 & . & . \\
\hline Ranunculus polyanthemophyllus & E1 & . & . & . & . & 8 & . & · \\
\hline Scabiosa hladnikiana & E1 & . & . & . & . & . & 80 & 40 \\
\hline Centaurea pannonica & E1 & . & . & . & . & . & 40 & . \\
\hline Euphrasia stricta & E1 & . & . & . & . & . & 20 & . \\
\hline Dorycnium germanicum & E1 & . & . & . & . & . & 10 & \\
\hline Linum catharticum & E1 & . & . & . & . & . & 10 & 30 \\
\hline Orchis tridentata & E1 & . & . & . & . & . & 10 & . \\
\hline Muscari comosum & E1 & . & . & . & . & . & 10 & . \\
\hline Filipendula vulgaris & E1 & . & . & . & . & . & . & 40 \\
\hline Thymus praecox s. str. & E1 & . & . & . & . & . & . & 40 \\
\hline Anthyllis vulneraria & E1 & . & . & . & . & . & . & 40 \\
\hline Ranunculus bulbosus & E1 & . & . & . & . & . & . & 20 \\
\hline Thymus longicaulis & E1 & . & . & . & . & . & . & 30 \\
\hline Genista tinctoria & E1 & . & . & . & . & . & . & 10 \\
\hline Erysimum odoratum & E1 & . & . & . & . & . & . & 10 \\
\hline
\end{tabular}




\begin{tabular}{|c|c|c|c|c|c|c|c|c|c|}
\hline & Successive number & & 1 & 2 & 3 & 4 & 5 & 6 & 7 \\
\hline & Anacamptis pyramidalis & E1 & . & . & . & . & . & . & 10 \\
\hline & Orchis ustulata & E1 & . & . & . & . & . & . & 10 \\
\hline & Scabiosa triandra & E1 & . & . & . & . & . & . & 20 \\
\hline & Scorzonera austriaca & E1 & . & . & . & . & . & . & 10 \\
\hline & Gentiana cruciata & E1 & . & . & . & . & . & . & 10 \\
\hline \multirow[t]{44}{*}{ TG } & Trifolio-Geranietea & & & & & & & & \\
\hline & Laserpitium siler & E1 & 100 & 92 & 50 & 100 & 46 & 50 & 100 \\
\hline & Laserpitium latifolium (subsp. asperum) & E1 & 68 & 83 & 100 & 63 & 8 & 50 & 60 \\
\hline & Thalictrum minus & E1 & 68 & 58 & 81 & 38 & 38 & 70 & . \\
\hline & Lilium carniolicum & E1 & 61 & 42 & 62 & . & . & . & . \\
\hline & Polygonatum odoratum & E1 & 55 & 33 & 73 & 50 & 23 & 60 & 30 \\
\hline & Silene nutans & E1 & 48 & 17 & 42 & 25 & 8 & 40 & 60 \\
\hline & Iris graminea & E1 & 45 & 50 & 96 & . & 15 & 20 & . \\
\hline & Arabis pauciflora & E1 & 42 & 92 & 58 & . & . & . & . \\
\hline & Tanacetum corymbosum agg. (T. clusii ?) & E1 & 29 & 67 & 69 & . & . & . & 40 \\
\hline & Vicia sylvatica & E1 & 29 & 25 & 54 & . & 8 & . & . \\
\hline & Anthericum ramosum & E1 & 26 & 8 & 42 & . & 31 & 90 & 30 \\
\hline & Libanotis sibirica subsp. montana & E1 & 26 & 67 & 4 & . & 31 & . & . \\
\hline & Valeriana collina $(V$. wallrothii) & E1 & 23 & 8 & 35 & 63 & 8 & 20 & 20 \\
\hline & Vincetoxicum hirundinaria & E1 & 23 & 8 & 65 & 25 & 54 & 90 & . \\
\hline & Verbascum lanatum & E1 & 19 & 25 & 58 & . & 8 & . & . \\
\hline & Clinopodium vulgare & E1 & 16 & . & 35 & 25 & 8 & 10 & . \\
\hline & Trifolium rubens & E1 & 16 & 58 & 85 & . & 15 & . & . \\
\hline & Origanum vulgare & E1 & 10 & 17 & 8 & 13 & . & 20 & . \\
\hline & Orobanche laserpitii-sileris & E1 & 10 & . & . & . & . & . & . \\
\hline & Achillea distans & E1 & 6 & 33 & 88 & . & 15 & . & . \\
\hline & Viola hirta & E1 & 6 & . & 12 & 13 & . & . & 20 \\
\hline & Thesium bavarum & E1 & 3 & 25 & 77 & 13 & 8 & 60 & 50 \\
\hline & Digitalis grandiflora & E1 & 3 & 17 & 15 & 13 & 8 & 10 & . \\
\hline & Hypericum perforatum & E1 & 3 & 8 & 8 & . & 8 & . & 40 \\
\hline & Agrimonia eupatoria & E1 & 3 & . & . & . & . & . & . \\
\hline & Geranium sanguineum & E1 & . & 25 & 65 & . & . & 50 & 50 \\
\hline & Verbascum lychnitis & E1 & . & 17 & . & . & . & . & . \\
\hline & Trifolium medium & E1 & . & 8 & 4 & . & 8 & . & . \\
\hline & Campanula rapunculoides & E1 & . & . & 19 & . & . & . & . \\
\hline & Hypericum hirsutum & E1 & . & . & 4 & . & . & 10 & 20 \\
\hline & Inula spiraeifolia & E1 & . & . & . & . & 8 & . & . \\
\hline & Libanotis daucifolia & E1 & . & . & . & . & 8 & . & . \\
\hline & Tephroseris longifolia & E1 & . & . & . & . & . & 20 & 30 \\
\hline & Verbascum austriacum & E1 & . & . & . & . & . & 20 & 20 \\
\hline & Lilium bulbiferum & E1 & . & . & . & . & . & 50 & . \\
\hline & Vicia cassubica & E1 & . & . & . & . & . & 50 & . \\
\hline & Lembotropis nigricans & E1 & . & . & . & . & . & 40 & . \\
\hline & Verbascum nigrum & E1 & . & . & . & . & . & 20 & . \\
\hline & Coronilla coronata & E1 & . & . & . & . & . & 10 & . \\
\hline & Trifolium alpestre & E1 & . & . & . & . & . & 10 & . \\
\hline & Dianthus croaticus & E1 & . & . & . & . & . & . & 70 \\
\hline & Melampyrum nemorosum & E1 & . & . & . & . & . & . & 10 \\
\hline & Centaurea stenolepis & E1 & . & . & . & . & . & . & 10 \\
\hline \multirow[t]{2}{*}{ PoT } & Poo alpinae-Trisetalia & & & & & & & & \\
\hline & Festuca nigrescens & E1 & 58 & 67 & 77 & 13 & 15 & . & . \\
\hline
\end{tabular}




\begin{tabular}{|c|c|c|c|c|c|c|c|c|c|}
\hline & Successive number & & 1 & 2 & 3 & 4 & 5 & 6 & 7 \\
\hline & Astrantia major & E1 & 39 & 17 & . & . & 8 & 10 & . \\
\hline & Traunsteinera globosa & E1 & 39 & 25 & 31 & . & 8 & . & . \\
\hline & Trollius europaeus & E1 & 32 & 75 & 4 & . & 38 & . & . \\
\hline & Pimpinella major subsp. rubra & E1 & 23 & 33 & . & 13 & . & . & . \\
\hline & Ranunculus nemorosus & E1 & 13 & 58 & 54 & 25 & 15 & 40 & . \\
\hline & Anthoxanthum odoratum & E1 & 13 & 58 & 50 & . & . & . & . \\
\hline & Crocus albiflorus & E1 & 13 & 25 & 15 & . & . & . & . \\
\hline & Campanula scheuchzeri & E1 & 13 & . & . & . & 38 & . & . \\
\hline & Poa alpina & E1 & 3 & . & . & . & . & . & . \\
\hline & Polygonum bistorta & E1 & 3 & . & . & . & . & . & . \\
\hline & Agrostis capillaris & E1 & . & 17 & . & . & 38 & 10 & . \\
\hline & Cerastium fontanum & E1 & . & . & . & . & 8 & . & . \\
\hline & Dactylorhiza majalis & E1 & . & . & . & . & . & . & 20 \\
\hline \multirow[t]{33}{*}{ MA } & Molinio-Arrhenatheretea & & & & & & & & \\
\hline & Lotus corniculatus s. lat. & E1 & 45 & 50 & 81 & 38 & . & 60 & 60 \\
\hline & Galium album (inc. G. mollugo) & E1 & 35 & 58 & 81 & . & 15 & 20 & 20 \\
\hline & Dactylis glomerata s.str. & E1 & 32 & 50 & 27 & . & 23 & 20 & 30 \\
\hline & Leontodon hispidus subsp. hispidus & E1 & 23 & 17 & 12 & . & 8 & 20 & 30 \\
\hline & Trifolium pratense & E1 & 13 & 17 & 4 & 25 & 15 & . & 20 \\
\hline & Lathyrus pratensis & E1 & 10 & 42 & 12 & 25 & 23 & 60 & 50 \\
\hline & Vicia sepium & E1 & 6 & 8 & . & . & . & . & 10 \\
\hline & Veronica chamaedrys & E1 & 3 & 8 & . & 13 & . & 30 & . \\
\hline & Achillea millefolium agg. & E1 & 3 & . & . & 13 & . & 10 & 20 \\
\hline & Angelica sylvestris & E1 & . & 17 & . & . & 31 & . & . \\
\hline & Achillea roseoalba & E1 & . & 8 & . & . & . & . & . \\
\hline & Tragopogon pratensis subsp. orientalis & E1 & . & . & 8 & . & 8 & . & 10 \\
\hline & Vicia cracca & E1 & . & . & . & 13 & 8 & . & . \\
\hline & Orobanche minor & E1 & . & . & . & 13 & . & . & . \\
\hline & Festuca rubra & E1 & . & . & . & . & 8 & 100 & 60 \\
\hline & Rumex acetosa & E1 & . & . & . & . & 8 & 20 & 10 \\
\hline & Deschampsia cespitosa & E1 & . & . & . & . & 8 & . & . \\
\hline & Stellaria graminea & E1 & . & . & . & . & 8 & . & 10 \\
\hline & Leucanthemum praecox et $L$. ircutianum & E1 & . & . & . & . & . & 30 & 10 \\
\hline & Prunella vulgaris & E1 & . & . & . & . & . & 20 & 60 \\
\hline & Trisetum flavescens & E1 & . & . & . & . & . & 20 & 10 \\
\hline & Arrhenatherum elatius & E1 & . & . & . & . & . & 20 & . \\
\hline & Poa pratensis & E1 & . & . & . & . & . & 10 & . \\
\hline & Geranium phaeum & E1 & . & . & . & . & . & 10 & · \\
\hline & Centaurea jacea & E1 & . & . & . & . & . & . & 40 \\
\hline & Plantago lanceolata & E1 & . & . & . & . & . & . & 30 \\
\hline & Ranunculus acris & E1 & . & . & . & . & . & . & 20 \\
\hline & Colchicum autumnale & E1 & . & . & . & . & . & . & 20 \\
\hline & Taraxacum officinale agg. & E1 & . & . & . & . & . & . & 10 \\
\hline & Trifolium campestre & E1 & . & . & . & . & . & . & 10 \\
\hline & Helictotrichon pubescens & E1 & . & . & . & . & . & . & 10 \\
\hline & Veronica serpyllifolia & E1 & . & . & . & . & . & . & 10 \\
\hline \multirow[t]{5}{*}{$\mathrm{CU}$} & Calluno-Ulicetea & & & & & & & & \\
\hline & Phyteuma zahlbruckneri & E1 & 19 & . & 50 & 25 & 8 & . & . \\
\hline & Carex pallescens & E1 & 3 & . & . & . & . & . & . \\
\hline & Luzula multiflora & E1 & 3 & 8 & . & . & . & . & . \\
\hline & Potentilla erecta & E1 & . & 33 & 77 & 13 & 15 & 70 & 70 \\
\hline
\end{tabular}




\begin{tabular}{|c|c|c|c|c|c|c|c|c|c|}
\hline & Successive number & & 1 & 2 & 3 & 4 & 5 & 6 & 7 \\
\hline & Arnica montana & E1 & . & 8 & . & . & . & . & . \\
\hline & Carex pilulifera & E1 & . & 8 & . & . & . & . & . \\
\hline & Galium pumilum & E1 & . & . & 35 & . & . & . & . \\
\hline & Calluna vulgaris & E1 & . & . & 4 & . & . & . & . \\
\hline & Genista germanica & E1 & . & . & 4 & . & . & . & . \\
\hline & Coeloglossum viride & E1 & . & . & 4 & . & . & . & . \\
\hline & Danthonia decumbens & E1 & . & . & . & . & 8 & . & . \\
\hline \multicolumn{10}{|c|}{ MuA Mulgedio-Aconitetea } \\
\hline & Silene vulgaris subsp. antelopum & E1 & 58 & 42 & . & 75 & . & . & . \\
\hline & Lathyrus occidentalis var. montanus (inc. L. laevigatus) & E1 & 32 & 42 & 19 & 13 & . & . & . \\
\hline & Aconitum lycoctonum s. lat. & E1 & 29 & 17 & 4 & . & 54 & 10 & . \\
\hline & Hypericum maculatum & E1 & 23 & 58 & 27 & 13 & 23 & . & . \\
\hline & Polygonatum verticillatum & E1 & 19 & 33 & 12 & . & 23 & . & . \\
\hline & Veratrum album s. lat. & E1 & 19 & 42 & 4 & . & 62 & . & . \\
\hline & Pleurospermum austriacum & E1 & 13 & 42 & 65 & . & 8 & . & . \\
\hline & Rumex arifolius & E1 & 13 & 50 & . & 13 & 8 & . & . \\
\hline & Salix appendiculata & E2 & 13 & . & . & . & 8 & . & . \\
\hline & Geranium sylvaticum & E1 & 10 & . & . & . & . & . & . \\
\hline & Heracleum sphondylium subsp. montanum (inc. subsp. elegans) & E1 & 10 & 33 & 12 & 38 & 23 & . & . \\
\hline & Allium victorialis & E1 & 6 & 8 & . & . & . & . & . \\
\hline & Centaurea montana & E1 & 6 & . & . & . & . & . & 30 \\
\hline & Aconitum angustifolium (inc. A. tauricum) & E1 & 3 & 8 & . & . & 15 & . & . \\
\hline & Chaerophyllum villarsii & E1 & 3 & 17 & . & . & . & . & . \\
\hline & Eryngium alpinum & E1 & 3 & 8 & . & . & . & . & . \\
\hline & Myrrhis odorata & E1 & 3 & 17 & . & . & . & . & . \\
\hline & Senecio ovatus & E1 & 3 & 17 & 23 & . & 15 & . & . \\
\hline & Sorbus chamaemespilus & E2 & 3 & 8 & . & . & . & . & . \\
\hline & Thalictrum aquilegiifolium & E1 & 3 & 25 & 8 & 13 & . & . & 30 \\
\hline & Urtica dioica & E1 & 3 & . & . & . & . & . & . \\
\hline & Carduus carduelis & E1 & . & 25 & . & . & . & . & . \\
\hline & Crepis pyrenaica & E1 & . & 17 & . & . & . & . & . \\
\hline & Poa hybrida & E1 & . & 17 & . & . & . & . & . \\
\hline & Silene dioica & E1 & . & 17 & . & . & . & . & . \\
\hline & Aconitum degenii subsp. paniculatum & E1 & . & 8 & 4 & . & . & . & . \\
\hline & Geum rivale & E1 & . & 8 & . & . & 8 & . & . \\
\hline & Ranunculus platanifolius & E1 & . & 8 & . & . & 8 & . & . \\
\hline & Pedicularis hacquetii & E1 & . & 8 & . & . & . & . & . \\
\hline & Chaerophyllum aureum & E1 & . & . & 4 & . & . & . & . \\
\hline & Grafia golaka & E1 & . & . & . & . & 100 & 100 & . \\
\hline & Molopospermum peloponnesiacum subsp. bauhinii & E1 & . & . & . & . & 31 & . & . \\
\hline & Alchemilla xanthochlora & E1 & . & . & . & . & 8 & . & . \\
\hline & Rumex alpinus & E1 & . & . & . & . & 8 & . & . \\
\hline & Salix glabra & E1 & . & . & . & . & 8 & . & . \\
\hline & Stemmacantha rhapontica & E1 & . & . & . & . & 8 & . & . \\
\hline & Dactylis glomerata subsp. slovenica & E1 & . & . & . & . & 8 & . & . \\
\hline & Cirsium waldsteinii & E1 & . & . & . & . & . & 30 & . \\
\hline \multicolumn{10}{|c|}{ SCF Scheuchzerio-Caricetea fuscae } \\
\hline & Gentiana utriculosa & E1 & 3 & . & . & . & . & . & 30 \\
\hline & Parnassia palustris & E1 & 3 & . & . & . & . & . & . \\
\hline & Pinguicula alpina & E1 & 3 & . & . & . & . & . & . \\
\hline
\end{tabular}




\begin{tabular}{|c|c|c|c|c|c|c|c|c|c|}
\hline & Successive number & & 1 & 2 & 3 & 4 & 5 & 6 & 7 \\
\hline \multirow[t]{11}{*}{ TR } & Thlaspietea rotundifolii & & & & & & & & \\
\hline & Ligusticum seguieri & E1 & 32 & 25 & 62 & . & . & . & . \\
\hline & Biscutella laevigata & E1 & 19 & . & 23 & 25 & . & 40 & . \\
\hline & Viola pyrenaica & E1 & 16 & 8 & . & . & . & . & . \\
\hline & Adenostyles glabra & E1 & 6 & 8 & . & . & 8 & . & . \\
\hline & Rumex scutatus & E1 & 6 & . & . & . & . & . & . \\
\hline & Astrantia carniolica & E1 & 3 & . & . & . & 8 & . & . \\
\hline & Petasites paradoxus & E1 & 3 & . & . & . & 8 & . & . \\
\hline & Leontodon hispidus subsp. hyoseroides & E1 & 3 & . & . & . & . & . & . \\
\hline & Silene vulgaris subsp. glareosa & E1 & 3 & . & . & . & . & . & . \\
\hline & Euphorbia triflora subsp. kerneri & E1 & . & . & . & 13 & . & . & . \\
\hline \multirow[t]{19}{*}{ AT } & Asplenietea trichomanis & & & & & & & & \\
\hline & Primula auricula & E1 & 19 & 25 & 12 & . & . & . & . \\
\hline & Saxifraga hostii & E1 & 16 & 8 & . & 13 & . & . & . \\
\hline & Saxifraga crustata & E1 & 10 & 8 & . & . & . & . & . \\
\hline & Dianthus sylvestris & E1 & 6 & 8 & . & . & . & . & 50 \\
\hline & Iris pallida subsp. cengialti & E1 & 6 & 8 & . & . & . & . & . \\
\hline & Sedum album & E1 & 6 & . & . & . & . & . & . \\
\hline & Sedum maximum & E1 & 6 & 8 & 12 & . & . & . & . \\
\hline & Asplenium ruta-muraria & E1 & 6 & 8 & . & . & . & . & . \\
\hline & Silene hayekiana & E1 & 3 & 8 & . & . & . & . & . \\
\hline & Carex brachystachys & E1 & 3 & . & . & . & . & . & . \\
\hline & Festuca stenantha & E1 & 3 & . & . & . & . & . & . \\
\hline & Rhamnus pumilus & E1 & 3 & . & . & . & . & . & . \\
\hline & Asplenium trichomanes & E1 & 3 & . & . & . & . & . & . \\
\hline & Sempervivum tectorum & E1 & . & 8 & . & . & . & . & . \\
\hline & Athamantha turbith & E1 & . & . & 4 & . & . & . & . \\
\hline & Campanula spicata & E1 & . & . & . & 13 & . & . & . \\
\hline & Valeriana saxatilis & E1 & . & . & . & . & . & 10 & . \\
\hline & Hieracium glaucum & E1 & . & . & . & . & . & . & 10 \\
\hline \multirow[t]{20}{*}{ EP } & Erico-Pinetea & & & & & & & & \\
\hline & Chamaecytisus hirsutus s. lat. & E1 & 71 & 50 & 54 & . & . & 80 & 10 \\
\hline & Genista radiata & E1 & 68 & 25 & . & 63 & 8 & . & . \\
\hline & Calamagrostis varia & E1 & 61 & 17 & 4 & 88 & 38 & 100 & . \\
\hline & Erica carnea & E1 & 26 & 25 & 62 & 63 & 8 & 100 & 20 \\
\hline & Peucedanum austriacum subsp. rablense & E1 & 42 & 25 & 4 & 75 & 8 & . & . \\
\hline & Carex ornithopoda & E1 & 10 & 8 & 23 & . & . & . & . \\
\hline & Aquilegia nigricans (inc. A. vulgaris) & E1 & 10 & . & . & . & . & 20 & 30 \\
\hline & Rubus saxatilis & E1 & 6 & 17 & 12 & 13 & 38 & 20 & . \\
\hline & Polygala chamaebuxus & E1 & 6 & 17 & 4 & 13 & . & 60 & 10 \\
\hline & Molinia caerulea subsp. arundinacea & E1 & 3 & 8 & 19 & 50 & 15 & 50 & 20 \\
\hline & Leontodon incanus & E1 & 3 & . & . & . & . & . & 70 \\
\hline & Cotoneaster tomentosus & E2 & 3 & . & . & . & . & . & . \\
\hline & Juniperus sibirica & E2 & 3 & . & . & . & . & . & . \\
\hline & Pinus mugo & E2 & 3 & . & . & . & . & . & . \\
\hline & Chamaecytisus purpureus & E1 & . & . & . & 38 & 15 & . & . \\
\hline & Daphne cneorum & E1 & . & . & . & 25 & . & . & . \\
\hline & Arctostaphylos uva-ursi & E1 & . & . & . & 13 & . & . & . \\
\hline & Knautia ressmannii & E1 & . & . & . & 13 & . & . & . \\
\hline & Carex alba & E1 & . & . & . & . & 8 & . & . \\
\hline
\end{tabular}




\begin{tabular}{|c|c|c|c|c|c|c|c|c|c|}
\hline & Successive number & & 1 & 2 & 3 & 4 & 5 & 6 & 7 \\
\hline & Genista januensis & E1 & . & . & . & . & . & . & 10 \\
\hline \multirow[t]{16}{*}{ VP } & Vaccinio-Piceetea & & & & & & & & \\
\hline & Picea abies & E2 & 32 & 17 & 8 & . & . & . & . \\
\hline & Aposeris foetida & E1 & 23 & 58 & 38 & . & . & 30 & . \\
\hline & Calamagrostis arundinacea & E1 & 6 & 25 & 88 & . & 31 & . & . \\
\hline & Luzula luzuloides & E1 & 3 & 17 & 27 & . & 23 & . & . \\
\hline & Rosa pendulina & E2 & 3 & 8 & 31 & 13 & 15 & 40 & . \\
\hline & Solidago virgaurea & E1 & . & 17 & . & . & . & 10 & . \\
\hline & Clematis alpina & E1 & . & 8 & . & . & . & . & . \\
\hline & Vaccinium myrtillus & E1 & . & 8 & . & . & . & . & . \\
\hline & Veronica urticifolia & E1 & . & . & 15 & . & . & . & . \\
\hline & Maianthemum bifolium & E1 & . & . & 4 & . & . & . & . \\
\hline & Larix decidua & E1 & . & . & . & 13 & . & . & . \\
\hline & Gentiana asclepiadea & E1 & . & . & . & . & 31 & . & . \\
\hline & Hieracium sylvaticum & E1 & . & . & . & . & . & 20 & 10 \\
\hline & Homogyne sylvestris & E1 & . & . & . & . & . & 20 & . \\
\hline & Valeriana tripteris & E1 & . & . & . & . & . & 10 & . \\
\hline \multirow[t]{5}{*}{$\mathrm{EC}$} & Erythronio-Carpinion & & & & & & & & \\
\hline & Ornithogalum pyrenaicum & E1 & 39 & 25 & 19 & . & . & . & . \\
\hline & Primula vulgaris & E1 & 16 & 8 & 27 & . & . & 40 & 40 \\
\hline & Galanthus nivalis & E1 & 3 & 8 & . & . & . & . & . \\
\hline & Helleborus odorus & E1 & . & 25 & . & . & 15 & 10 & . \\
\hline \multirow[t]{10}{*}{$\mathrm{AF}$} & Aremonio-Fagion & & & & & & & & \\
\hline & Knautia drymeia & E1 & 48 & 92 & 58 & . & 62 & 70 & 40 \\
\hline & Cyclamen purpurascens & E1 & 32 & . & 15 & 13 & . & 100 & 20 \\
\hline & Rhamnus fallax & E2 & 10 & . & . & . & . & . & . \\
\hline & Dentaria enneaphyllos & E1 & 3 & . & . & . & . & . & 10 \\
\hline & Anemone trifolia & E1 & . & . & . & 13 & . & . & . \\
\hline & Helleborus niger & E1 & . & . & . & . & . & 20 & . \\
\hline & Omphalodes verna & E1 & . & . & . & . & . & 20 & . \\
\hline & Hacquetia epipactis & E1 & . & . & . & . & . & 20 & . \\
\hline & Euphorbia carniolica & E1 & . & . & . & . & . & 20 & . \\
\hline \multirow[t]{19}{*}{ FS } & Fagetalia sylvaticae & & & & & & & & \\
\hline & Mercurialis perennis & E1 & 71 & 75 & 58 & 50 & 31 & 10 & 10 \\
\hline & Melica nutans & E1 & 19 & . & . & . & . & . & . \\
\hline & Symphytum tuberosum & E1 & 16 & . & 58 & . & 8 & . & . \\
\hline & Fagus sylvatica & E2 & 10 & . & 15 & . & . & 10 & . \\
\hline & Lilium martagon & E1 & 10 & 33 & 23 & . & 38 & . & . \\
\hline & Myosotis sylvatica & E1 & 10 & 17 & . & . & . & . & . \\
\hline & Luzula nivea & E1 & 6 & . & . & 25 & . & . & . \\
\hline & Euphorbia amygdaloides & E1 & 3 & . & . & . & . & . & 10 \\
\hline & Galium laevigatum & E1 & 3 & 8 & 31 & 13 & . & . & . \\
\hline & Lathyrus vernus & E1 & 3 & . & 8 & . & 15 & . & . \\
\hline & Poa nemoralis & E1 & 3 & . & . & 13 & . & . & 10 \\
\hline & Salvia glutinosa & E1 & 3 & . & . & . & . & 10 & 10 \\
\hline & Scrophularia nodosa & E1 & 3 & . & . & . & . & . & . \\
\hline & Tilia cordata & E2 & 3 & . & . & . & . & . & . \\
\hline & Acer pseudoplatanus & E1 & 3 & 8 & 4 & . & 8 & . & 20 \\
\hline & Hesperis candida & E1 & 3 & . & . & . & . & . & . \\
\hline & Campanula trachelium & E1 & . & 8 & . & . & . & . & . \\
\hline & Lonicera alpigena & E2 & . & 8 & . & . & . & . & . \\
\hline
\end{tabular}




\begin{tabular}{|c|c|c|c|c|c|c|c|c|c|}
\hline & Successive number & & 1 & 2 & 3 & 4 & 5 & 6 & 7 \\
\hline & Prenanthes purpurea & E1 & . & . & 12 & . & . & 10 & . \\
\hline & Melica nutans & E1 & . & . & 4 & . & 8 & 60 & . \\
\hline & Heracleum sphondylium subsp. sphondylium & E1 & . & . & 4 & . & 23 & . & . \\
\hline & Daphne mezereum & E1 & . & . & 4 & . & 8 & . & . \\
\hline & Festuca heterophylla & E1 & . & . & 4 & . & . & . & 40 \\
\hline & Neottia nidus-avis & E1 & . & . & 4 & . & . & . & . \\
\hline & Luzula nivea & E1 & . & . & . & . & 15 & . & . \\
\hline & Fraxinus excelsior & E1 & . & . & . & . & 8 & . & . \\
\hline & Galeobdolon flavidum & E1 & . & . & . & . & 8 & . & . \\
\hline & Laburnum alpinum & E1 & . & . & . & . & 8 & . & . \\
\hline & Phyteuma spicatum & E1 & . & . & . & . & . & 10 & 20 \\
\hline & Epipactis helleborine & E1 & . & . & . & . & . & 10 & . \\
\hline & Galeobdolon montanum & E1 & . & . & . & . & . & 10 & . \\
\hline & Ranunculus lanuginosus & E1 & . & . & . & . & . & . & 10 \\
\hline & Euphorbia dulcis & E1 & . & . & . & . & . & . & 10 \\
\hline \multirow[t]{19}{*}{ QP } & Quercetalia pubescentis & & & & & & & & \\
\hline & Peucedanum schottii var. schottii & E1 & 42 & 25 & 85 & . & 15 & . & . \\
\hline & Convallaria majalis & E1 & 13 & 17 & 65 & 13 & 31 & 100 & 10 \\
\hline & Primula veris subsp. columnae & E1 & 13 & 25 & 92 & . & . & . & . \\
\hline & Carex flacca & E1 & 10 & 8 & 58 & 38 & 15 & 30 & 70 \\
\hline & Sorbus aria & E2 & 10 & 17 & 46 & . & . & . & . \\
\hline & Melittis melissophyllum & E1 & 6 & 25 & 81 & . & . & 30 & . \\
\hline & Ostrya carpinifolia & E2 & 3 & . & 15 & . & . & . & . \\
\hline & Orchis mascula subsp. speciosa & E1 & . & 17 & 12 & . & . & . & . \\
\hline & Peucedanum schottii var. petraeum & E1 & . & 8 & . & . & . & . & . \\
\hline & Fraxinus ornus & E1 & . & . & 11 & . & . & 10 & . \\
\hline & Hypericum montanum & E1 & . & . & 8 & . & . & . & . \\
\hline & Arabis turrita & E1 & . & . & 4 & . & . & . & . \\
\hline & Mercurialis ovata & E1 & . & . & . & . & . & 100 & 40 \\
\hline & Clematis recta & E1 & . & . & . & . & . & 10 & 30 \\
\hline & Campanula persicifolia & E1 & . & . & . & . & . & 10 & 20 \\
\hline & Acer obtusatum & E1 & . & . & . & . & . & . & 20 \\
\hline & Tamus communis & E1 & . & . & . & . & . & . & 10 \\
\hline & Quercus pubescens & E1 & . & . & . & . & . & . & 10 \\
\hline \multirow[t]{17}{*}{ QF } & Querco-Fagetea & & & & & & & & \\
\hline & Anemone nemorosa & E1 & 16 & 8 & 27 & . & . & 20 & 20 \\
\hline & Carex digitata & E1 & 16 & 17 & 15 & 13 & . & 40 & 50 \\
\hline & Listera ovata & E1 & 13 & 8 & 8 & . & . & . & . \\
\hline & Corylus avellana & E2 & 3 & 17 & 8 & . & . & . & · \\
\hline & Cruciata glabra & E1 & 3 & 25 & . & . & 15 & 30 & 50 \\
\hline & Dactylorhiza fuchsii & E1 & 3 & 8 & 27 & . & 15 & . & · \\
\hline & Veratrum nigrum & E1 & . & 25 & . & . & . & . & · \\
\hline & Cephalanthera longifolia & E1 & . & . & 42 & . & . & . & . \\
\hline & Carex montana & E1 & . & . & 38 & . & . & 30 & 70 \\
\hline & Chamaecytisus supinus & E1 & . & . & 4 & . & . & . & . \\
\hline & Populus tremula & E1 & . & . & 4 & . & . & . & . \\
\hline & Platanthera bifolia & E1 & . & . & 4 & . & . & . & . \\
\hline & Crategus monogyna & E1 & . & . & 4 & . & . & . & . \\
\hline & Viola riviniana & E1 & . & . & 4 & . & . & . & . \\
\hline & Serratula tinctoria subsp. tinctoria & E1 & . & . & . & . & 38 & 20 & . \\
\hline & Festuca gigantea & E1 & . & . & . & . & 8 & . & . \\
\hline
\end{tabular}




\begin{tabular}{|c|c|c|c|c|c|c|c|c|c|}
\hline & Successive number & & 1 & 2 & 3 & 4 & 5 & 6 & 7 \\
\hline & Betonica officinalis & E1 & . & . & . & . & . & 50 & 50 \\
\hline & Hepatica nobilis & E1 & . & . & . & . & . & 40 & . \\
\hline & Melampyrum pratense & E1 & . & . & . & . & . & . & 10 \\
\hline & Euphorbia angulata & E1 & . & . & . & . & . & . & 10 \\
\hline \multirow[t]{5}{*}{$\mathrm{RP}$} & Rhamno-Prunetea & & & & & & & & \\
\hline & Rosa glauca & E2 & 3 & . & 8 & . & . & . & . \\
\hline & Rosa canina & E1 & 3 & . & . & . & . & . & . \\
\hline & Rhamnus catharticus & E2a & . & 8 & . & . & . & . & . \\
\hline & Rubus fruticosus & E2 & . & . & . & . & . & . & 10 \\
\hline \multirow[t]{6}{*}{$\mathrm{O}$} & Other species & & & & & & & & \\
\hline & Rubus idaeus & E2 & 10 & . & 19 & . & 54 & . & . \\
\hline & Juniperus communis & E2 & 6 & 8 & . & . & . & . & . \\
\hline & Fragaria vesca & E1 & 3 & . & 15 & 13 & . & 80 & . \\
\hline & Hieracium sp. & E1 & 3 & . & . & . & . & . & . \\
\hline & Poa sp. & E1 & 3 & . & . & . & . & . & . \\
\hline \multirow[t]{5}{*}{ SM } & Myosotis arvensis & E1 & 3 & . & . & . & . & . & . \\
\hline & Sorbus aucuparia & E1 & . & . & 8 & . & . & . & . \\
\hline & Potentilla recta & E1 & . & . & . & . & 15 & . & . \\
\hline & Salix eleagnos & E1 & . & . & . & . & 8 & . & . \\
\hline & Orobanche sp. & E1 & . & . & . & . & . & . & 10 \\
\hline \multirow[t]{5}{*}{ M } & Mosses & & & & & & & & \\
\hline & Tortella tortuosa & E0 & 10 & 17 & . & . & . & . & . \\
\hline & Schistidium apocarpum & E0 & 3 & 8 & . & . & . & . & . \\
\hline & Fissidens dubius & E0 & . & 8 & . & . & . & . & . \\
\hline & Polytrichum formosum & E0 & . & . & . & . & . & . & . \\
\hline
\end{tabular}

1: Centaureo julici-Laserpitietum sileris ass. nov. hoc loco typicum - the Julian Alps, Slovenia

2: Centaureo julici-Laserpitietum sileris ass. nov. hoc loco typicum var. Brachypodium rupestre - the Julian Alps, Slovenia

3: Centaureo julici-Laserpitietum sileris ass. nov. hoc loco asphodeletosum albi subass. nov. hoc loco - the Julian Alps, Slovenia (Dakskobler et al. 2007, table 1, rel. 8-33)

4: Centaureo julici-Laserpitietum sileris ass. nov. hoc loco var. geogr. Pedicularis elongata, W Julian Alps, the Carnic Alps, NE Italy

5: Laserpitio sileri-Grafietum golakae ass. nov. hoc loco, the Julian Prealps, NE Italy

6: Scabioso hladnikianae-Grafietum golakae Čarni 2007 - central Slovenia - Polhograjsko hribovje (Čarni 2007, Table 1)

7: Laserpitietum sileris Springer 1987 var. geogr. Dianthus croaticus - southeastern Slovenia - Gorjanci (Čarni et al. 2005, Tab. 1, rel. 47-56) 
Table 6: Phytosociological groups in the tall herb and fringe communities with the dominant Laserpitium siler or Grafia golaka in NE Italy and Slovenia (relative frequencies).

Tabela 6: Fitocenološke skupine v združbah visokih steblik in gozdnih robov z dominantnima vrstama Laserpitium siler ali Grafia golaka v severovzhodni Italiji in Sloveniji (relativne frekvence).

\begin{tabular}{|c|c|c|c|c|c|c|c|}
\hline Successive number (Zaporedna številka) & 1 & 2 & 3 & 4 & 5 & 6 & 7 \\
\hline Number of relevés (Število popisov) & 31 & 12 & 26 & 8 & 13 & 10 & 10 \\
\hline Sign for the syntaxa (Oznaka sintakosnov) & CJLS1 & CJLS2 & CJLSaa & CJLSLP & GgLs & ShGg & Ls \\
\hline Caricion austroalpinae & 6.49 & 6.41 & 5.30 & 3.72 & 2.56 & 0.41 & 0.00 \\
\hline Elyno-Seslerietea & 24.10 & 19.50 & 9.87 & 26.81 & 6.97 & 6.91 & 2.37 \\
\hline Festuco-Brometea & 20.22 & 19.50 & 22.75 & 22.73 & 19.49 & 27.03 & 41.23 \\
\hline Trifolio-Geranietea & 14.04 & 14.80 & 22.04 & 13.02 & 13.72 & 17.68 & 15.64 \\
\hline Poo alpinae-Trisetalia & 4.76 & 6.27 & 3.05 & 1.51 & 6.33 & 1.22 & 0.47 \\
\hline Molinio-Arrhenatheretea & 3.28 & 4.60 & 3.79 & 4.13 & 6.14 & 9.15 & 13.27 \\
\hline Calluno-Ulicetea & 0.49 & 0.97 & 3.77 & 1.12 & 1.17 & 1.42 & 1.66 \\
\hline Mulgedio-Aconitetea & 5.33 & 9.60 & 3.06 & 4.87 & 16.06 & 2.85 & 1.42 \\
\hline Scheuchzerio-Caricetea fuscae & 0.19 & 0.00 & 0.00 & 0.00 & 0.00 & 0.00 & 0.71 \\
\hline Thlaspietea rotundifolii & 1.79 & 0.70 & 1.43 & 1.12 & 0.90 & 0.81 & 0.00 \\
\hline Asplenietea trichomanis & 1.79 & 1.53 & 0.47 & 0.77 & 0.00 & 0.20 & 1.42 \\
\hline Erico-Pinetea & 6.10 & 3.20 & 3.06 & 13.40 & 5.20 & 8.74 & 4.03 \\
\hline Vaccinio-Piceetea & 1.30 & 2.65 & 3.55 & 0.77 & 3.77 & 2.64 & 0.24 \\
\hline Erythronio-Carpinion & 1.11 & 1.11 & 0.77 & 0.00 & 0.57 & 1.02 & 0.95 \\
\hline Aremonio-Fagion & 1.79 & 1.53 & 1.23 & 0.77 & 2.34 & 5.08 & 1.66 \\
\hline Fagetalia sylvaticae & 3.28 & 2.65 & 3.86 & 2.98 & 6.71 & 2.64 & 3.32 \\
\hline Quercetalia pubescentis & 1.86 & 2.37 & 8.03 & 1.51 & 2.30 & 5.89 & 4.98 \\
\hline Querco-Fagetea & 1.05 & 1.81 & 3.12 & 0.38 & 2.86 & 4.67 & 6.16 \\
\hline Rhamno-Prunetea & 0.12 & 0.14 & 0.13 & 0.00 & 0.00 & 0.00 & 0.24 \\
\hline Other species (Druge vrste) & 0.56 & 0.14 & 0.71 & 0.38 & 2.90 & 1.63 & 0.24 \\
\hline Mosses (Mahovi) & 0.25 & 0.55 & 0.00 & 0.00 & 0.00 & 0.00 & 0.00 \\
\hline Total (Skupaj) & 100 & 100 & 100 & 100 & 100 & 100 & 100 \\
\hline
\end{tabular}

1: Centaureo julici-Laserpitietum sileris ass. nov. hoc loco typicum - the Julian Alps, Slovenia

2: Centaureo julici-Laserpitietum sileris ass. nov. hoc loco typicum var. Brachypodium rupestre - the Julian Alps, Slovenia

3: Centaureo julici-Laserpitietum sileris ass. nov. hoc loco asphodeletosum albi subass. nov. hoc loco - the Julian Alps, Slovenia (Dakskobler et al. 2007, table 1, rel. 8-33)

4: Centaureo julici-Laserpitietum sileris ass. nov. hoc loco var. geogr. Pedicularis elongata, W Julian Alps, the Carnic Alps, NE Italy

5: Laserpitio sileri-Grafietum golakae ass. nov. hoc loco, the Julian Prealps, NE Italy

6: Scabioso hladnikianae-Grafietum golakae Čarni 2007 - central Slovenia - Polhograjsko hribovje (Čarni 2007, Table 1)

7: Laserpitietum sileris Springer 1987 var. geogr. Dianthus croaticus - southeastern Slovenia - Gorjanci (Čarni et al. 2005, Tab. 1, rel. 47-56) 
Table 7: Two stands with Grafia golaka on its classical locality under the Mt. Golaki (the Trnovski gozd plateau, W Slovenia).

Tabela 7: Dva sestoja z vrsto Grafia golaka na njenem klasičnem nahajališču pod Golaki (Trnovski gozd, zahodna Slovenija).

\begin{tabular}{|c|c|c|c|c|c|}
\hline & Number of relevé & & 1 & 2 & \\
\hline & Working number of relevé & & 241068 & 241069 & \\
\hline & Altitude in $\mathrm{m}$ & & 1390 & 1400 & \\
\hline & Aspect & & SW & SSE & \\
\hline & Slope in degrees & & 30 & 30 & \\
\hline & Parent material & & A & A & \\
\hline & Soil & & $\mathrm{Li}$ & $\mathrm{Li}$ & \\
\hline & Stoniness in $\%$ & & 90 & 50 & \\
\hline & Cover of tree layer in $\%$ & E3 & 5 & . & \\
\hline & Cover of shrub layer in $\%$ & E2 & 40 & . & \\
\hline & Cover of herb layer in $\%$ & E1 & 20 & 50 & \\
\hline & Number of species & & 33 & 22 & \\
\hline & Relevé area & $\mathrm{m}^{2}$ & 200 & 100 & \\
\hline & Date of taking relevé & & $6 / 21 / 2011$ & $6 / 21 / 2011$ & \\
\hline & Locality & & Veliki Golak & Veliki Golak & \\
\hline & Quadrant & & $0049 / 1$ & 0049/1 & \\
\hline & Coordinate Y (Gauß-Krüger, D 48) & $\mathrm{m}$ & 413255 & 413174 & \\
\hline & Coordinate X (Gauß-Krüger, D 48) & $\mathrm{m}$ & 5093056 & 5093092 & \\
\hline \multirow[t]{6}{*}{ MuA } & Mulgedio-Aconitetea & & & & Pr. \\
\hline & Grafia golaka & E1 & 2 & 4 & 2 \\
\hline & Salix appendiculata & $\mathrm{E} 2 \mathrm{~b}$ & 3 & . & 1 \\
\hline & Salix appendiculata & $\mathrm{E} 2 \mathrm{a}$ & 1 & + & 2 \\
\hline & Geranium sylvaticum & E1 & + & + & 2 \\
\hline & Thalictrum aquilegiifolium & E1 & . & + & 1 \\
\hline \multirow[t]{2}{*}{ TG } & Trifolio-Geranietea & & & & \\
\hline & Laserpitium latifolium & E1 & + & 2 & 2 \\
\hline \multirow[t]{2}{*}{ ES } & Elyno-Seslerietea & & & & \\
\hline & Betonica alopecuros & E1 & + & + & 2 \\
\hline \multirow[t]{2}{*}{$\mathrm{CA}$} & Gentiana lutea subsp. symphyandra & E1 & + & . & 1 \\
\hline & Scabiosa lucida subsp. stricta & E1 & + & . & 1 \\
\hline \multirow[t]{4}{*}{ FB } & Festuco-Brometea & & & & \\
\hline & Buphthalmum salicifolium & E1 & + & 1 & 2 \\
\hline & Cirsium erisithales & E1 & + & + & 2 \\
\hline & Carlina acaulis & E1 & . & + & 1 \\
\hline \multirow[t]{5}{*}{$\mathrm{EP}$} & Erico-Pinetea & & & & \\
\hline & Calamagrostis varia & E1 & + & 1 & 2 \\
\hline & Rubus saxatilis & E1 & + & + & 2 \\
\hline & Rhododendron hirsutum & $\mathrm{E} 2 \mathrm{a}$ & 3 & . & 1 \\
\hline & Erica carnea & E1 & + & . & 1 \\
\hline \multirow[t]{4}{*}{$\mathrm{AF}$} & Aremonio-Fagion & & & & \\
\hline & Cyclamen purpurascens & E1 & + & + & 2 \\
\hline & Omphalodes verna & E1 & + & . & 1 \\
\hline & Knautia drymeia & E1 & . & 1 & 1 \\
\hline \multirow[t]{6}{*}{ FS } & Fagetalia sylvaticae & & & & \\
\hline & Symphytum tuberosum & E1 & + & + & 2 \\
\hline & Acer pseudoplatanus & $\mathrm{E} 2 \mathrm{a}$ & + & . & 1 \\
\hline & Acer pseudoplatanus & $\mathrm{E} 2 \mathrm{~b}$ & + & . & 1 \\
\hline & Acer pseudoplatanus & E1 & . & + & 1 \\
\hline & Actaea spicata & E1 & + & . & 1 \\
\hline
\end{tabular}




\begin{tabular}{|c|c|c|c|c|c|}
\hline & Number of relevé & & 1 & 2 & Pr. \\
\hline & Fagus sylvatica & E2a & + & . & 1 \\
\hline & Lonicera alpigena & $\mathrm{E} 2 \mathrm{a}$ & + & . & 1 \\
\hline & Tilia platyphyllos & E3 & $\mathrm{r}$ & . & 1 \\
\hline & Viola reichenbachiana & E1 & + & . & 1 \\
\hline & Dryopteris filix-mas & E1 & $\mathrm{r}$ & . & 1 \\
\hline & Lilium martagon & E1 & . & + & 1 \\
\hline & Melica nutans & E1 & . & + & 1 \\
\hline & Phyteuma spicatum subsp. coeruleum & E1 & . & + & 1 \\
\hline \multirow[t]{2}{*}{ QP } & Quercetalia pubescentis & & & & \\
\hline & Melittis melissophyllum & E1 & + & + & 2 \\
\hline \multirow[t]{2}{*}{ QF } & Querco-Fagetea & & & & \\
\hline & Anemone nemorosa & E1 & . & 1 & 1 \\
\hline \multirow[t]{4}{*}{ VP } & Vaccinio-Piceetea & & & & \\
\hline & Rosa pendulina & $\mathrm{E} 2 \mathrm{a}$ & 1 & . & 1 \\
\hline & Gentiana asclepiadea & E1 & + & . & 1 \\
\hline & Picea abies & E3 & + & . & 1 \\
\hline \multirow[t]{3}{*}{ TR } & Thlaspietea rotundifolii & & & & \\
\hline & Adenostyles glabra & E1 & 1 & + & 2 \\
\hline & Hieracium bifidum & E1 & $\mathrm{r}$ & . & 1 \\
\hline \multirow[t]{3}{*}{ AT } & Asplenieta trichomanis & & & & \\
\hline & Paederota lutea & E1 & 1 & 1 & 2 \\
\hline & Asplenium ruta-muraria & E1 & + & . & 1 \\
\hline \multirow[t]{3}{*}{ M } & Mosses & & & & \\
\hline & Ctenidium molluscum & E0 & + & . & 1 \\
\hline & Tortella tortuosa & E0 & + & . & 1 \\
\hline
\end{tabular}
A Limestone
Li Lithosol 